\title{
Polynomial Bounds for the Grid-Minor Theorem
}

\author{
CHANDRA CHEKURI, University of Illinois, Urbana-Champaign \\ JULIA CHUZHOY, Toyota Technological Institute at Chicago
}

\begin{abstract}
One of the key results in Robertson and Seymour's seminal work on graph minors is the grid-minor theorem (also called the excluded grid theorem). The theorem states that for every grid $H$, every graph whose treewidth is large enough relative to $|V(H)|$ contains $H$ as a minor. This theorem has found many applications in graph theory and algorithms. Let $f(k)$ denote the largest value such that every graph of treewidth $k$ contains a grid minor of size $(f(k) \times f(k))$. The best previous quantitative bound, due to recent work of Kawarabayashi and Kobayashi, and Leaf and Seymour, shows that $f(k)=\Omega(\sqrt{\log k / \log \log k})$. In contrast, the best known upper bound implies that $f(k)=O(\sqrt{k / \log k})$. In this article, we obtain the first polynomial relationship between treewidth and grid minor size by showing that $f(k)=\Omega\left(k^{\delta}\right)$ for some fixed constant $\delta>0$, and describe a randomized algorithm, whose running time is polynomial in $|V(G)|$ and $k$, that with high probability finds a model of such a grid minor in $G$.
\end{abstract}

Categories and Subject Descriptors: Mathematics of Computing [Discrete Nathematics]: Graph TheoryGraph Algorithms

General Terms: Algorithms, Theory

Additional Key Words and Phrases: Excluded grid theorem, graph minor theory

ACM Reference Format:

Chandra Chekuri and Julia Chuzhoy. 2016. Polynomial bounds for the grid-minor theorem. J. ACM 63, 5, Article 40 (December 2016), 65 pages.

DOI: http://dx.doi.org/10.1145/2820609

\section{INTRODUCTION}

The seminal work of Robertson and Seymour on graph minors makes essential use of the notions of tree decompositions and treewidth. A key structural result in their work is the grid-minor theorem (also called the excluded grid theorem), which states that for every grid $H$, every graph whose treewidth is large enough relative to $|V(H)|$ contains $H$ as a minor. This theorem has found many applications in graph theory and algorithms. Let $f(k)$ denote the largest value such that every graph of treewidth $k$ contains a grid minor of size $(f(k) \times f(k))$. The quantitative estimate for $f$ given in the original proof of Robertson and Seymour [1986] was substantially improved by Robertson et al. [1994], who showed that $f(k)=\Omega\left(\log ^{1 / 5} k\right)$; see Diestel et al. [1999] and

The work of the first author was supported in part by NSF grants CCF-1016684 and CCF-1319376, and by TTI Chicago during a sabbatical visit in fall 2013. The work of the second author was supported in part by NSF CAREER grant CCF-0844872, and Sloan Research Fellowship and NSF grant CCF-1318242.

Authors' addresses: C. Chekuri, Department of Computer Science, University of Illinois, 3228 Siebel Center, 201 N. Goodwin Ave, Urbana, IL 61801; email: chekuri@illinois.edu; J. Chuzhoy, Toyota Technological Institute at Chicago,6045 S. Kenwood Ave., Chicago IL 60637; email: cjulia@ttic.edu.

Permission to make digital or hard copies of part or all of this work for personal or classroom use is granted without fee provided that copies are not made or distributed for profit or commercial advantage and that copies show this notice on the first page or initial screen of a display along with the full citation. Copyrights for components of this work owned by others than ACM must be honored. Abstracting with credit is permitted. To copy otherwise, to republish, to post on servers, to redistribute to lists, or to use any component of this work in other works requires prior specific permission and/or a fee. Permissions may be requested from Publications Dept., ACM, Inc., 2 Penn Plaza, Suite 701, New York, NY 10121-0701 USA, fax +1 (212) 869-0481, or permissions@acm.org.

(c) 2016 ACM 0004-5411/2016/12-ART40 $\$ 15.00$

DOI: http://dx.doi.org/10.1145/2820609 

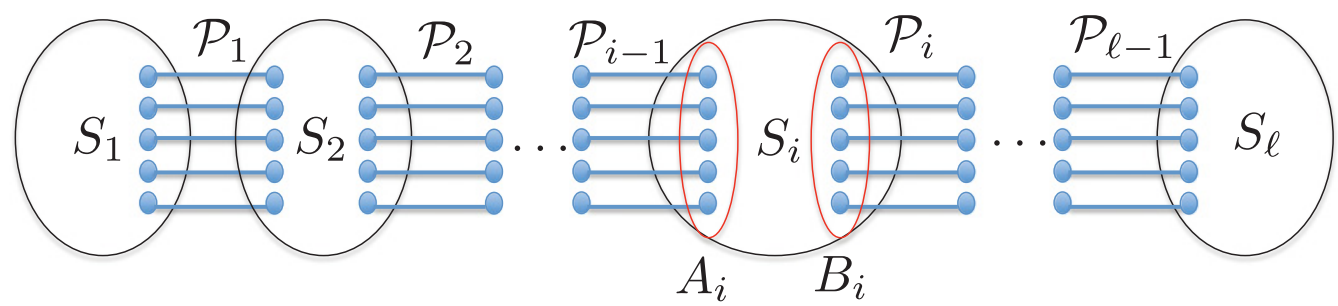

Fig. 1. Path-of-sets system of width $w$ and length $\ell$. Each set $\mathcal{P}_{i}$ contains $w$ paths. All paths in $\bigcup_{i=1}^{\ell-1} \mathcal{P}_{i}$ are node disjoint and internally disjoint from $\bigcup_{i=1}^{\ell} S_{i}$.

Diestel [2012] for a simpler proof with a slightly weaker bound. There have been recent improvements by Kawarabayashi and Kobayashi [2012], and by Leaf and Seymour [2015], giving the best previous bound of $f(k)=\Omega(\sqrt{\log k / \log \log k})$. On the other hand, the known upper bounds on $f$ are polynomial in $k$. It is easy to see, for example by considering the complete graph on $n$ nodes, whose treewidth is $n-1$, that $f(k)=O(\sqrt{k})$. This can be slightly improved to $f(k)=O(\sqrt{k / \log k})$ by considering sparse random graphs (or $\Omega(\log n$ )-girth constant-degree expanders) [Robertson et al. 1994]. Robertson et al. [1994] suggest that this value may be sufficient, and Demaine et al. [2009] conjecture that the bound of $f(k)=\Theta\left(k^{1 / 3}\right)$ is both necessary and sufficient. It has been an important open problem to prove a polynomial relationship between a graph's treewidth and the size of the largest grid minor in it. In this article, we prove the following theorem, which accomplishes this goal, while also giving a polynomial-time randomized algorithm to find a model of the grid minor. Given a function $f: \mathbb{Z}^{+} \rightarrow \mathbb{Z}^{+}$, we say that $f(m)=O(\operatorname{poly}(m))$ if $f(m)=O\left(m^{c}\right)$ for some constant $c$ independent of $m$. Similarly, we say that $f(m)=O($ poly $\log m)$ if $f(m)=O\left(\log ^{c} m\right)$ for some constant $c>0$ independent of $m$. We use notation $\Omega($ poly $m$ ) and $\Omega$ (poly $\log m$ ) analogously.

THEOREM 1.1. There is a universal constant $\delta>0$, such that for every $k \geq 1$, every graph $G$ of treewidth $k$ contains a grid of size $\left(\Omega\left(k^{\delta} /\right.\right.$ poly $\left.\left.\log k\right) \times \Omega\left(k^{\delta} / \operatorname{poly} \log k\right)\right)$ as a minor. Moreover, there is a randomized algorithm that, given $G$, with high probability outputs a model of the grid minor in time $O(\operatorname{poly}(|V(G)| \cdot k))$.

Our proof shows that $\delta$ is at least $1 / 98$ in the preceding theorem. We note that the relationship between grid minors and treewidth is much tighter in some special classes of graphs. In planar graphs $f(k)=\Omega(k)$ [Robertson et al. 1994]; a similar linear relationship is known in bounded-genus graphs [Demaine et al. 2005] and graphs that exclude a fixed graph $H$ as a minor [Demaine and Hajiaghayi 2008] (see also Kawarabayashi and Kobayashi [2012]).

We obtain the following corollary by observing that every simple planar graph $H$ is a minor of a grid of size $\left(k^{\prime} \times k^{\prime}\right)$ for $k^{\prime}=O(|V(H)|)$ [Robertson et al. 1994].

COROLlary 1.2. There is a universal constant $c$ such that, if $G$ excludes a simple planar graph $H$ as a minor, then the treewidth of $G$ is $O\left(|V(H)|^{c}\right)$.

The grid-minor-theorem has several important applications in graph theory and algorithms, and also in proving lower bounds. The quantitative bounds in some of these applications can be directly improved by our main theorem. We anticipate that there will be other applications for our main theorem, as well as for the algorithmic and graph-theoretic tools that we develop here.

Our proof and algorithm are based on a combinatorial object, called a path-of-sets system, that we informally describe now (Figure 1). A path-of-sets system of width $w$ and length $\ell$ consists of a collection of $\ell$ disjoint sets of nodes $S_{1}, \ldots, S_{\ell}$ together with 
collections of paths $\mathcal{P}_{1}, \ldots, \mathcal{P}_{\ell-1}$ that are disjoint, which connect the sets in a path-like fashion. The number of paths in each set $\mathcal{P}_{i}$ is $w$. Moreover, for each $i$, the induced graph $G\left[S_{i}\right]$ satisfies the following connectivity properties for the endpoints of the paths $\mathcal{P}_{i-1}$ and $\mathcal{P}_{i}$ (sets $A_{i}$ and $B_{i}$ of vertices in the figure): for every pair $A \subseteq A_{i}, B \subseteq B_{i}$ of vertex subsets with $|A|=|B|$, there are $|A|$ node-disjoint paths connecting $A$ to $B$ in $G\left[S_{i}\right]$.

Given a path-of-sets system of width $w$ and length $w$, we can efficiently find a model of a grid minor of size $\left(\Omega\left(w^{1 / 2}\right) \times \Omega\left(w^{1 / 2}\right)\right)$ in $G$, slightly strengthening a similar recent result of Leaf and Seymour [2015], who use a related combinatorial object that they refer to as a $(w, \ell)$-grill. Our main contribution is to show that there is a randomized algorithm that, given a graph $G$ of treewidth $k$, with high probability constructs a pathof-sets system of width $w$ and length $w$ in $G$ if $w^{c} \leq O(k / \operatorname{polylog}(\mathrm{k}))$, where $c$ is a fixed constant. The running time of the algorithm is polynomial in $|V(G)|$ and $k$. The central ideas for the construction build on and extend recent work on approximation algorithms for the maximum edge-disjoint paths problem with constant congestion [Chuzhoy 2016; Chuzhoy and Li 2016] and connections to treewidth [Chekuri and Ene 2013; Chekuri and Chuzhoy 2013]. To construct the path-of-sets system, we use a closely related object, called a tree-of-sets system. The definition of the tree-of-sets system is quite similar to the definition of the path-of-sets system except instead of connecting the clusters $S_{i}$ into a single long path, we connect them into a tree whose maximum vertex degree is at most 3. We extend and strengthen the results of Chuzhoy [2016], Chuzhoy and Li [2016], and Chekuri and Ene [2013] by showing an efficient randomized algorithm that, given a graph of treewidth $k$, with high probability constructs a large tree-of-sets system. We then show how to construct a large path-of-sets system given a large treeof-sets system. We believe that the tree-of-sets system is an interesting combinatorial object of independent interest and hope that future work will yield simpler and faster algorithms for constructing it, as well as improved parameters. This could lead to improvements in algorithms for related routing problems.

Subsequent work. Building on this work, the authors recently showed in Chekuri and Chuzhoy [2015] an efficient randomized algorithm that, given any graph $G$ of treewidth $k$, with high probability produces a topological minor $H$ of $G$ (called a treewidth sparsifier), whose treewidth is $\Omega(k /$ poly $\log k)$, maximum vertex degree is 3 , and $|V(H)|=O\left(k^{4}\right.$ poly $\left.\log k\right)$.

More recently, Chuzhoy [2015] has improved our bound on $\delta$ in Theorem 1.1, proving the theorem for $\delta=1 / 36$, using a different construction of the path-of-sets system. By combining some results and techniques from this work with this new construction, she further improved the constant $\delta$ to $1 / 19$. Her results use the treewidth sparsifier from Chekuri and Chuzhoy [2015] as a starting point. We note that her proof is nonconstructive and does not provide an algorithm to find a model of the grid minor (although it is likely that it can be turned into an algorithm whose running time is polynomial in $n$ and exponential in $k$ ).

\section{PRELIMINARIES}

In this article, we use the term efficient algorithm to refer to a (possibly randomized) algorithm that runs in time polynomial in the length of its input.

All graphs in this article are finite, and they do not have loops. We say that a graph is simple to indicate that it does not have parallel edges; otherwise, parallel edges are allowed. Given a graph $G=(V, E)$ and a set $A \subseteq V$ of its vertices, we denote by out ${ }_{G}(A)$ the set of all edges with exactly one endpoint in $A$ and by $E_{G}(A)$ the set of all edges with both endpoints in $A$. For disjoint sets of vertices $A$ and $B$, the set of edges with one endpoint in $A$ and the other in $B$, is denoted by $E_{G}(A, B)$. For a vertex $v \in V$, we denote the degree of $v$ by $d_{G}(v)$. We may omit the subscript $G$ if it is clear from the context. 
Given a set $\mathcal{P}$ of paths in $G$, we denote by $V(\mathcal{P})$ the set of all vertices participating in paths in $\mathcal{P}$, and similarly $E(\mathcal{P})$ is the set of all edges that participate in paths in $\mathcal{P}$. We sometimes refer to sets of vertices as clusters. All logarithms are to the base of 2 . We say that an event $\mathcal{E}$ holds with high probability if the probability of $\mathcal{E}$ is at least $1-1 / n^{c}$ for some constant $c>1$, where $n$ is the cardinality of vertex set of the graph in question. We use the following simple claims several times.

ClaIm 2.1. There is an efficient algorithm that, given a set $\left\{x_{1}, \ldots, x_{n}\right\}$ of nonnegative integers, with $\sum_{i} x_{i}=N$, and $x_{i} \leq 2 N / 3$ for all $i$, computes a partition $(A, B)$ of $\{1, \ldots, n\}$, such that $\sum_{i \in A} x_{i} \geq N / 3$ and $\sum_{i \in B} x_{i} \geq N / 3$.

Proof. We assume without loss of generality that $x_{1} \geq x_{2} \geq \cdots \geq x_{n}$ and process the integers in this order. When $x_{i}$ is processed, we add $i$ to $A$ if $\sum_{j \in A} x_{j} \leq \sum_{j \in B} x_{j}$, and we add it to $B$ otherwise. We claim that at the end of this process, $\sum_{i \in A} x_{i}, \sum_{i \in B} x_{i} \geq N / 3$. Indeed, 1 is always added to $A$. If $x_{1} \geq N / 3$, then, since $x_{1} \leq 2 N / 3$, it is easy to see that both subsets of integers sum up to at least $N / 3$. Otherwise, $\left|\sum_{i \in A} x_{i}-\sum_{i \in B} x_{i}\right| \leq$ $\max _{i}\left\{x_{i}\right\} \leq x_{1} \leq N / 3$.

ClaIm 2.2. Let $T$ be a rooted tree, and $\ell, p \geq 1$ integers, such that $|V(T)| \geq \ell p$. Then either $T$ has at least $\ell$ leaves or there is a root-to-leaf path containing at least $p$ vertices in $T$.

Proof. Suppose that $T$ has fewer than $\ell$ leaves, and each root-to-leaf path has fewer than $p$ vertices. Then, since every node belongs to some root-to-leaf path of $T$, $|V(T)|<\ell p$, contradicting our assumption.

The treewidth of a graph $G=(V, E)$ is typically defined via tree decompositions. A tree decomposition of a graph $G$ consists of a tree $T=(V(T), E(T))$ and a collection of vertex sets $\left\{X_{v} \subseteq V\right\}_{v \in V(T)}$ called bags such that the following two properties are satisfied: (i) for each edge $(a, b) \in E$, there is some node $v \in V(T)$ with both $a, b \in X_{v}$, and (ii) for each vertex $a \in V$, the set of all nodes of $T$ whose bags contain $a$ induces a nonempty (connected) subtree of $T$. The width of a given tree decomposition is $\max _{v \in V(T)}\left|X_{v}\right|-1$, and the treewidth of a graph $G$, denoted by $\operatorname{tw}(G)$, is the width of a minimum-width tree decomposition of $G$.

We say that a simple graph $H$ is a minor of a graph $G$ if $H$ can be obtained from $G$ by a sequence of edge deletion, vertex deletion, and edge contraction operations. Equivalently, a simple graph $H$ is a minor of $G$ if there is a map $\varphi$, assigning to each vertex $v \in V(H)$ a subset $\varphi(v)$ of vertices of $G$, and to each edge $e=(u, v) \in E(H)$ a path $\varphi(e)$ connecting a vertex of $\varphi(u)$ to a vertex of $\varphi(v)$, such that

-for every vertex $v \in V(H)$, the subgraph of $G$ induced by $\varphi(v)$ is connected;

-if $u, v \in V(H)$ and $u \neq v$, then $\varphi(u) \cap \varphi(v)=\emptyset$; and

- the paths in set $\{\varphi(e) \mid e \in E(H)\}$ are internally node disjoint, and they are internally disjoint from $\bigcup_{v \in V(H)} \varphi(v)$.

A map $\varphi$ satisfying these conditions is called a model of $H$ in $G$. (We note that this definition is slightly different from the standard one, requiring that for each $e \in E(H)$, path $\varphi(e)$ consists of a single edge; but it is immediate to verify that both definitions are equivalent, and it is more convenient for us to work with the preceding definition.) For convenience, we may sometimes refer to the map $\varphi$ as the embedding of $H$ into $G$, and specifically to $\varphi(v)$ and $\varphi(e)$ as the embeddings of the vertex $v \in V(H)$ and the edge $e \in E(H)$, respectively.

The $(g \times g)$-grid is a graph whose vertex set is $\{v(i, j) \mid 1 \leq i, j \leq g\}$. The edge set consists of two subsets: a set of horizontal edges $E_{1}=\{(v(i, \bar{j}), v(i, \bar{j}+1)) \mid 1 \leq i \leq g$; $1 \leq j<g\}$ and a set of vertical edges $E_{2}=\{(v(i, j), v(i+1, j)) \mid 1 \leq i<g ; 1 \leq j \leq g\}$. 
The subgraph induced by $E_{1}$ consists of $g$ disjoint paths, which we call the rows of the grid; the $i$ th row is the row incident with $v(i, 1)$. Similarly, the subgraph induced by $E_{2}$ consists of $g$ disjoint paths, which we call the columns of the grid; the $j$ th column is the column incident with $v(1, j)$. We say that graph $G$ contains a $(g \times g)$-grid minor if some minor $H$ of $G$ is isomorphic to the $(g \times g)$-grid.

\subsection{Flows and Cuts}

In this section, we define standard single-commodity flows and discuss their relationships with the corresponding notions of cuts. Most definitions and results from this section can be found in standard textbooks; we refer the reader to Schrijver [2003] for more details.

Let $G=(V, E)$ be an edge-capacitated graph with $c(e)>0$ denoting the capacity of edge $e \in E$. Given two disjoint vertex subsets $S, T \subseteq V$, let $\mathcal{P}$ be the set of all paths that start at $S$ and terminate at $T$. An $S$ - $T$ flow $f: \mathcal{P} \rightarrow \mathbb{R}_{+}$is an assignment of nonnegative values to paths in $\mathcal{P}$. The value of the flow is $\sum_{P \in \mathcal{P}} f(P)$. Given a flow $f$, for each edge $e \in E$, we define a flow through $e$ to be $f^{\prime}(e)=\sum_{P \in \mathcal{P}: e \in P} f(P)$. The edge congestion of the flow is $\max _{e \in E}\left\{f^{\prime}(e) / c(e)\right\}$. We say that the flow $f$ is valid, or that it causes no edge congestion, if its edge congestion is at most 1 . We note that even though $|\mathcal{P}|$ may be exponential in $|V|$, there are known efficient algorithms to compute a valid flow of a specified value $F$ (if it exists), and to compute a flow of maximum value. Moreover, in both cases, the number of paths in $\mathcal{P}$ with nonzero flow value $f(P)$ is guaranteed to be at most $|E|$. Such flows can be computed, for example, by using an equivalent edge-based flow formulation together with linear programming and a flowpath decomposition of the resulting solution (see Schrijver [2003] for more details). It is also well known that if all edge capacities are integral, then whenever a valid $S-T$ flow of an integral value $F$ exists in $G$, there is also a valid $S-T$ flow $\tilde{f}$ of the same value, where $\tilde{f}(P)$ is integral for all $P \in \mathcal{P}$, and the number of paths $P$ with $\tilde{f}(P)>0$ is at most $|E|$. Moreover, such a flow can be found efficiently. Throughout the article, whenever the edge capacities of a given graph $G$ are not specified, we assume that they are all unit.

A cut in a graph $G$ is a bipartition $(A, B)$ of its vertices, with $A, B \neq \emptyset$. We sometimes use $\bar{A}$ to denote $V \backslash A$. The value of the cut is the total capacity of all edges in $E(A, B)$ (if the edge capacities of $G$ are not specified, then the value of the cut is $|E(A, B)|$ ). We say that a cut $(A, B)$ separates $S$ from $T$ if $S \subseteq A$ and $T \subseteq B$. The well-known max-flow min-cut theorem states that for a graph $G$ and disjoint vertex subsets $S, T$, the value of the maximum $S$ - $T$ flow in $G$ is equal to the value of the minimum cut separating $S$ from $T$ in $G$. Notice that if all edges of $G$ have unit capacities, and the value of the maximum flow from $S$ to $T$ is $F$, then the maximum number of edge-disjoint paths connecting the vertices of $S$ to the vertices of $T$ is also $F$, and if $E^{\prime}$ is a minimum-cardinality set of edges, such that $G \backslash E^{\prime}$ contains no path connecting a vertex of $S$ to a vertex of $T$, then $\left|E^{\prime}\right|=F$. When $S=\{s\}$ and $T=\{t\}$, then we sometimes refer to the $S-T$ flow and $S-T$ cut as $s$ - $t$ flow and $s$ - $t$ cut, respectively.

Given a subset $\mathcal{P}^{\prime} \subseteq \mathcal{P}$ of paths connecting vertices of $S$ to vertices of $T$ in $G$, we say that the paths in $\mathcal{P}^{\prime}$ cause edge congestion at most $\eta$ if for every edge $e \in E$, the total number of paths in $\mathcal{P}^{\prime}$ containing $e$ is at most $\eta \cdot c(e)$.

A variant of the $S-T$ flow that we sometimes use is when the capacities are given on the graph vertices and not edges. Such a flow $f$ is defined exactly as before, except now for every vertex $v \in V$, we let $f^{\prime}(v)=\sum_{\substack{P \in \mathcal{P}_{i} \\ v \in P}} f(P)$, and we define the congestion of the flow to be $\max _{v \in V}\left\{f^{\prime}(v) / c(v)\right\}$. If the congestion of the flow is at most 1 , then we say that it is a valid flow, or that the flow causes no vertex congestion. When all vertex 
capacities are integral, there is a maximum flow $f$ such that all values $f(P)$ for all $P \in \mathcal{P}$ are integral. In particular, if all vertex capacities are 1 , and there is a valid $S$ - $T$ flow of value $F$, then there are $F$ node-disjoint paths connecting vertices of $S$ to vertices of $T$, and this set of paths can be found efficiently.

All of the preceding definitions and results about single-commodity flows carry over to directed graphs as well, except cuts are defined slightly differently. As before, a cut in $G$ is a bipartition $(A, B)$ of the vertices of $G$. The value of the cut is the total capacity of edges connecting vertices of $A$ to vertices of $B$. The max-flow min-cut theorem remains valid in directed graphs, with this definition of cuts. For every directed flow network, there exists a maximum $S$ - $T$ flow in which for every pair $\left(e, e^{\prime}\right)$ of antiparallel edges, at most one of these edges carries nonzero flow; if all edge capacities are integral, then there is a maximum flow that is integral and has this property. This follows from the equivalent edge-based definition of flows. Flows in directed graphs with capacities on vertices are defined similarly.

We will repeatedly use the following simple claim.

CLAIM 2.3. There is an efficient algorithm that, given a bipartite graph $G=\left(V_{1}, V_{2}, E\right)$ with maximum vertex degree at most $\Delta$, computes a matching $E^{\prime} \subseteq E$ of cardinality at least $|E| / \Delta$.

Proof. We set up a directed flow network: start with graph $G$, assign each of its vertices capacity 1 , and direct its edges from $V_{1}$ to $V_{2}$. Add a source $s$ of infinite capacity that connects to every vertex in $V_{1}$ with a directed edge, and add a destination vertex $t$ of infinite capacity to which every vertex of $V_{2}$ connects with a directed edge. It is immediate to see that this network has a valid $s$ - $t$ flow of value $|E| / \Delta$, by sending $1 / \Delta$ flow units on each edge $e \in E$. From the integrality of flow, there is a valid integral flow of the same value, which defines the desired matching.

\subsection{Sparsest Cut}

Suppose that we are given a graph $G=(V, E)$ and a subset $\mathcal{T} \subseteq V$ of $k$ vertices, called terminals. Given a cut $(S, \bar{S})$ in $G$ with $S \cap \mathcal{T}, \bar{S} \cap \mathcal{T} \neq \emptyset$, the sparsity of $(S, \bar{S})$ is $\Phi_{\mathcal{T}}(S, \bar{S})=\frac{|E(S, \bar{S})|}{\min \{|S \cap \mathcal{T}|,|\bar{S} \cap \mathcal{T}|\}}$, and the value of the sparsest cut in $G$ with respect to $\mathcal{T}$

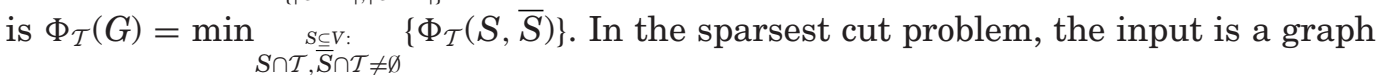
$G$ with a set $\mathcal{T}$ of terminals, and the goal is to find a cut of minimum sparsity. Arora et al. [2009] have shown an $O(\sqrt{\log k})$-approximation algorithm for the sparsest cut problem, where $k=|\mathcal{T}|$. We use $\mathcal{A}_{\mathrm{ARV}}$ to refer to their algorithm, and we denote by $\beta_{\mathrm{ARV}}(k)=O(\sqrt{\log k})$ its approximation factor. We will repeatedly use the following observation.

OBSERVATION 2.4. Let $G$ be a graph, and let $\mathcal{T} \subseteq V(G)$ a subset of its vertices called terminals, where $|\mathcal{T}|=k$ for some $k>0$. Assume further that for some $0<\alpha \leq 1$, $\Phi_{\mathcal{T}}(G) \geq \alpha$. Then for every pair $\mathcal{T}^{\prime}, \mathcal{T}^{\prime \prime} \subseteq \mathcal{T}$ of disjoint equal-size subsets of terminals, there is a flow $f$ in $G$, where every terminal in $\mathcal{T}^{\prime}$ sends one flow unit, every terminal in $\mathcal{T}^{\prime \prime}$ receives one flow unit, and the edge congestion is bounded by $1 / \alpha$.

Proof. Let $\mathcal{T}^{\prime}, \mathcal{T}^{\prime \prime} \subseteq \mathcal{T}$ be a pair of disjoint equal-size sets of terminals. We construct a directed flow network $H$ from $G$ by replacing each edge of $G$ with a pair of bi-directed edges and setting the capacity $c(e)$ of each such edge $e$ to be $1 / \alpha$. We then add two special vertices to the graph: the source $s$, which connects with a capacity- 1 edge to every vertex of $\mathcal{T}^{\prime}$, and the destination $t$, to which every vertex of $\mathcal{T}^{\prime \prime}$ connects with a capacity-1 edge. Let $k^{\prime}=\left|\mathcal{T}^{\prime}\right|=\left|\mathcal{T}^{\prime \prime}\right|$, and let $f$ be the maximum $s$ - $t$ flow in $H$. If the 
value of $f$ is at least $k^{\prime}$, then we can use $f$ to define a flow $f^{\prime}$, where every terminal in $\mathcal{T}^{\prime}$ sends one flow unit, every terminal in $\mathcal{T}^{\prime \prime}$ receives one flow unit, and the edge congestion is bounded by $1 / \alpha$ (as we can assume without loss of generality that for every pair $e^{\prime}, e^{\prime \prime}$ of antiparallel edges, only one of these edges carries nonzero flow). Therefore, we assume from now on that the value of $f$ is less than $k^{\prime}$. We will reach a contradiction by showing a cut whose sparsity with respect to $\mathcal{T}$ is less than $\alpha$.

Let $\left(A^{\prime}, B^{\prime}\right)$ be the minimum $s$ - $t$ cut in $H$, and let $E^{\prime}$ be the set of all edges of $H$ from $A^{\prime}$ to $B^{\prime}$, so $\sum_{e \in E^{\prime}} c(e)<k^{\prime}$. Let $A=A^{\prime} \backslash\{s\}$ and $B=B^{\prime} \backslash\{t\}$, and assume that $|\mathcal{T} \cap A| \leq|\mathcal{T} \cap B|$ 一the other case is symmetric. Let $k_{1}=\left|\mathcal{T}^{\prime} \cap A^{\prime}\right|$ and $k_{2}=\left|\mathcal{T}^{\prime} \cap B^{\prime}\right|$. Then $\sum_{e \in E^{\prime}} c(e) \geq k_{2}+\left|E_{G}(A, B)\right| / \alpha$. Therefore, $\left|E_{G}(A, B)\right|<\alpha\left(k^{\prime}-k_{2}\right)=\alpha k_{1}=\alpha\left|\mathcal{T}^{\prime} \cap A\right| \leq$ $\alpha|\mathcal{T} \cap A|$, and the cut $(A, B)$ has sparsity less than $\alpha$, a contradiction.

\subsection{Linkedness and Well-Linkedness}

We define the notion of linkedness and the different notions of well-linkedness that we use.

Definition 2.5. We say that a set $\mathcal{T}$ of vertices is $\alpha$-well-linked ${ }^{1}$ in $G$ if for every partition $(A, B)$ of the vertices of $G$ into two subsets, $|E(A, B)| \geq \alpha \cdot \min \{|A \cap \mathcal{T}|,|B \cap \mathcal{T}|\}$.

The following simple observation immediately follows from the definition of welllinkedness.

OBSERVATION 2.6. Let $G$ be a graph and $\mathcal{T} \subseteq V(G)$ a subset of its vertices, so that $\mathcal{T}$ is $\alpha$-well-linked in $G$, for some $0<\alpha \leq 1$. Then,

$-\Phi_{\mathcal{T}}(G) \geq \alpha$

-for every subset $\mathcal{T}^{\prime} \subseteq \mathcal{T}, \mathcal{T}^{\prime}$ is $\alpha$-well-linked in $G$; and

$-\mathcal{T}$ is $\alpha^{\prime}$-well-linked in $G$ for all $0<\alpha^{\prime}<\alpha$.

Definition 2.7. We say that a set $\mathcal{T}$ of vertices is node-well-linked in $G$ if for every pair $\left(\mathcal{T}_{1}, \mathcal{T}_{2}\right)$ of equal-size subsets of $\mathcal{T}$, there is a collection $\mathcal{P}$ of $\left|\mathcal{T}_{1}\right|$ node-disjoint paths connecting the vertices of $\mathcal{T}_{1}$ to the vertices of $\mathcal{T}_{2}$. (Note that $\mathcal{T}_{1}, \mathcal{T}_{2}$ are not necessarily disjoint, and we allow paths consisting of a single vertex.)

Definition 2.8. We say that two disjoint vertex subsets $A, B$ are linked in $G$ if for every pair of equal-size subsets $A^{\prime} \subseteq A, B^{\prime} \subseteq B$ there is a set $\mathcal{P}$ of $\left|A^{\prime}\right|=\left|B^{\prime}\right|$ node-disjoint paths connecting $A^{\prime}$ to $B^{\prime}$ in $G$.

Our algorithm starts with a graph $G$ of treewidth $k$ and then reduces its degree to $\operatorname{poly} \log (k)$ while preserving the treewidth to within a factor of poly $\log (k)$. As we show in the following, in bounded-degree graphs, the notions of edge- and node-welllinkedness are closely related to each other, and we exploit this connection throughout the algorithm.

THEOREM 2.9. Suppose that we are given a graph $G$ with maximum vertex degree at most $\Delta$, and two disjoint subsets $\mathcal{T}_{1}, \mathcal{T}_{2}$ of its vertices, such that $\mathcal{T}_{1} \cup \mathcal{T}_{2}$ is $\alpha$-well-linked in $G$ for some $0<\alpha \leq 1$, and each one of the sets $\mathcal{T}_{1}, \mathcal{T}_{2}$ is node-well-linked in $G$. Let $\mathcal{T}_{1}^{\prime} \subsetneq \mathcal{T}_{1}, \mathcal{T}_{2}^{\prime} \subsetneq \mathcal{T}_{2}$, be a pair of subsets with $\left|\mathcal{T}_{1}^{\prime}\right| \leq \frac{\alpha\left|\mathcal{T}_{1}\right|}{2 \Delta}$ and $\left|\mathcal{T}_{2}^{\prime}\right| \leq \frac{\alpha\left|\mathcal{T}_{2}\right|}{2 \Delta}$. Then $\mathcal{T}_{1}^{\prime}$ and $\mathcal{T}_{2}^{\prime}$ are linked in $G$.

\footnotetext{
${ }^{1}$ This notion of well-linkedness is based on edge cuts, and we distinguish it from node-well-linkedness that is directly related to treewidth. For technical reasons, it is easier to work with edge cuts, and hence we use the term well-linked to mean edge-well-linkedness and explicitly use the term node-well-linkedness when necessary.
} 
Proof. Let $\mathcal{T}=\mathcal{T}_{1} \cup \mathcal{T}_{2}$. We refer to the vertices of $\mathcal{T}$ as terminals. Denote $\left|\mathcal{T}_{1}\right|=\kappa_{1}$, $\left|\mathcal{T}_{2}\right|=\kappa_{2}$, and assume without loss of generality that $\kappa_{1} \leq \kappa_{2}$. Assume for contradiction that $\mathcal{T}_{1}^{\prime}$ and $\mathcal{T}_{2}^{\prime}$ are not linked in $G$. Then there are two sets $A \subseteq \mathcal{T}_{1}^{\prime}, B \subseteq \mathcal{T}_{2}^{\prime}$, with $|A|=|B|=\kappa^{\prime}$ for some $\kappa^{\prime} \leq \frac{\alpha \kappa_{1}}{2 \Delta}$, and a set $S$ of $\kappa^{\prime}-1$ vertices, separating $A$ from $B$ in $G$.

Let $A^{\prime} \subseteq \mathcal{T}_{1}$ be the set of all terminals $t \in \mathcal{T}_{1}$ such that $t$ lies in the same component of $G \backslash S$ as some vertex of $A$. We claim that $\left|A^{\prime}\right| \geq \kappa_{1}-\kappa^{\prime}$. Indeed, assume otherwise, and let $A^{\prime \prime} \subseteq \mathcal{T}_{1} \backslash A^{\prime}$ be a set of $\kappa^{\prime}$ vertices. Since $\overline{\mathcal{T}}_{1}$ is node-well-linked in $G$, there is a set $\mathcal{P}$ of $\kappa^{\prime}$ node-disjoint paths connecting the vertices of $A$ to the vertices of $A^{\prime \prime}$ in $G$. At most $\kappa^{\prime}-1$ of these paths may contain the vertices of $S$, and so at least one vertex of $\mathcal{T}_{1} \backslash A^{\prime}$ is connected to some vertex of $A$ in $G \backslash S$, a contradiction.

Similarly, we let $B^{\prime} \subseteq \mathcal{T}_{2}$ be the set of all terminals $t \in \mathcal{T}_{2}$ such that $t$ lies in the same component of $G \backslash S$ as some vertex of $B$. From the same arguments as earlier, $\left|B^{\prime}\right| \geq \kappa_{2}-\kappa^{\prime}$. Finally, we show that there is some pair $a \in A^{\prime}, b \in B^{\prime}$ of vertices that lie in the same connected component of $G \backslash S$. Indeed, since the terminals of $\mathcal{T}$ are $\alpha$-well-linked in $G$, there is a set $\mathcal{Q}$ of at least $\kappa_{1}-\kappa^{\prime}$ paths in $G$, where each path originates at a distinct vertex of $A^{\prime}$ and terminates at a distinct vertex of $B^{\prime}$, and every edge of $G$ participates in at most $1 / \alpha$ paths. At most $\left(\kappa^{\prime}-1\right) \Delta / \alpha$ of the paths in $\mathcal{Q}$ may contain the vertices of $S$. Since $|\mathcal{Q}|=\kappa_{1}-\kappa^{\prime}>\left(\kappa^{\prime}-1\right) \Delta / \alpha$, at least one path of $\mathcal{Q}$ belongs to $G \backslash S$. Therefore, there is a path in $G \backslash S$ from a vertex of $A$ to a vertex of $B$, a contradiction.

\subsection{Boosting Well-Linkedness}

Suppose that we are given a graph $G$ and a set $\mathcal{T}$ of vertices of $G$ called terminals, where $\mathcal{T}$ is $\alpha$-well-linked in $G$. Boosting theorems allow us to boost the well-linkedness by selecting an appropriate subset of the terminals, whose well-linkedness is greater than $\alpha$. We start with the following simple claim, which has been extensively used in past work to boost well-linkedness of terminals.

Claim 2.10. Suppose that we are given a graph $G$ and a set $\mathcal{T}$ of vertices of $G$, called terminals, such that $\mathcal{T}$ is $\alpha$-well-linked for some $0<\alpha<1$. Assume further that we are given a collection $\mathcal{S}$ of trees in $G$, and for every tree $T \in \mathcal{S}$ we are given a subset $\lambda_{T} \subseteq V(T) \cap \mathcal{T}$ of at least $\lceil 1 / \alpha\rceil$ terminals, such that for every pair $T \neq T^{\prime}$ of the trees, $\lambda_{T} \cap \lambda_{T^{\prime}}=\emptyset$. Assume further that each edge of $G$ belongs to at most $c$ trees, and let $\mathcal{T}^{\prime} \subseteq \mathcal{T}$ be a subset of terminals, containing exactly one terminal from each set $\lambda_{T}$ for $T \in \mathcal{S}$. Then $\mathcal{T}^{\prime}$ is $1 /(c+1)$-well-linked in $G$.

PRoOF. The proof provided here was suggested by an anonymous referee, and it is somewhat simpler than our original proof. Let $(A, B)$ be a partition of the vertices of $G$, and let $\mathcal{T}_{A}=\mathcal{T}^{\prime} \cap A, \mathcal{T}_{B}=\mathcal{T}^{\prime} \cap B$. Assume without loss of generality that $\left|\mathcal{T}_{A}\right| \leq\left|\mathcal{T}_{B}\right|$, and denote $\left|\mathcal{T}_{A}\right|=\kappa$. Our goal is to show that $|E(A, B)| \geq \kappa /(c+1)$. Assume for contradiction that $|E(A, B)|<\kappa /(c+1)$.

Let $\mathcal{S}_{1} \subseteq \mathcal{S}$ be the set of trees $T$ with $E(T) \cap E(A, B) \neq \emptyset$. Since each edge of $G$ belongs to at most $c$ trees, $\left|\mathcal{S}_{1}\right|<\kappa c /(c+1)$. Let $\mathcal{S}_{A} \subseteq \mathcal{S}$ be the set of all trees $T$ with $V(T) \cap \mathcal{T}_{A} \neq \emptyset$, and define $\mathcal{S}_{B} \subseteq \mathcal{S}$ similarly for $\mathcal{T}_{B}$. Then $\left|\mathcal{S}_{A}\right|,\left|\mathcal{S}_{B}\right| \geq \kappa$. Let $\mathcal{S}_{A}^{\prime}=\mathcal{S}_{A} \backslash \mathcal{S}_{1}$. Then $\left|\mathcal{S}_{A}^{\prime}\right|>\kappa-\kappa c /(c+1)=\kappa /(c+1)$, and every tree in $\mathcal{S}_{A}^{\prime}$ is contained in $A$. Similarly, let $\mathcal{S}_{B}^{\prime}=\mathcal{S}_{B} \backslash \mathcal{S}_{1}$, so $\left|\mathcal{S}_{B}^{\prime}\right|>\kappa /(c+1)$, and every tree in $\mathcal{S}_{B}^{\prime}$ is contained in $B$.

Let $\mathcal{T}_{A}^{\prime} \subseteq \mathcal{T} \cap A$ be the set of all terminals participating in the trees of $\mathcal{S}_{A}^{\prime}$, and define $\mathcal{T}_{B}^{\prime} \subseteq \mathcal{T} \cap \bar{B}$ similarly for $\mathcal{S}_{B}^{\prime}$. Then $\left|\mathcal{T}_{A}^{\prime}\right| \geq\lceil 1 / \alpha\rceil \cdot\left|\mathcal{S}_{1}^{\prime}\right| \geq \frac{\kappa}{\alpha(c+1)}$, and similarly $\left|\mathcal{T}_{B}^{\prime}\right| \geq \frac{\kappa}{\alpha(c+1)}$. From the $\alpha$-well-linkedness of the terminals in $\mathcal{T},|E(A, B)| \geq \alpha \cdot \min \left\{\left|\mathcal{T}_{A}^{\prime}\right|,\left|\mathcal{T}_{B}^{\prime}\right|\right\} \geq$ $\kappa /(c+1)$ must hold, a contradiction. 
This claim is already sufficient to boost the well-linkedness of a given set of terminals, as follows.

COROLlary 2.11. There is an efficient algorithm that, given a connected graph $G, a$ subset $\mathcal{T}$ of its vertices, such that for some $0<\alpha \leq 1, \mathcal{T}$ is $\alpha$-well-linked in $G$, and a partition $\mathcal{T}_{1}, \ldots, \mathcal{T}_{\ell}$ of $\mathcal{T}$, computes, for each $1 \leq i \leq \ell$ a subset $\mathcal{T}_{i}^{\prime} \subseteq \mathcal{T}_{i}$ of at least $\left\lfloor\frac{\left|\mathcal{T}_{i}\right|}{3\lceil 1 / \alpha\rceil}\right\rfloor$ vertices, so that $\bigcup_{i=1}^{\ell} \mathcal{T}_{i}^{\prime}$ is $1 / 2$-well-linked in $G$.

Proof. Throughout the proof, we refer to the vertices of $\mathcal{T}$ as terminals. For $1 \leq i \leq \ell$, denote $\left|\mathcal{T}_{i}\right|=\kappa_{i}$. We start with the following simple observation.

OBSERVATION 2.12. There is an efficient algorithm to compute a collection $\mathcal{F}$ of trees in $G$, and for every tree $T \in \mathcal{F}$, a subset $\lambda_{T} \subseteq V(T) \cap \mathcal{T}$ of its vertices, such that

- every edge of $G$ belongs to at most one tree;

- for every tree $T \in \mathcal{F},\lceil 1 / \alpha\rceil \leq\left|\lambda_{T}\right| \leq 3\lceil 1 / \alpha\rceil$; and

- the sets $\left\{\lambda_{T}\right\}_{T \in \mathcal{F}}$ define a partition of $\mathcal{T}$.

Proof. Let $T^{*}$ be a spanning tree of $G$ that we root at some vertex $r$. We perform several iterations, where in every iteration we delete some edges and vertices from $T^{*}$. For each vertex $v$ of the tree $T^{*}$, let $T_{v}^{*}$ denote the subtree rooted at $v$, and let $w\left(T_{v}^{*}\right)$ denote the total number of terminals in $T_{v}^{*}$. We build the set $\mathcal{F}$ of the trees gradually. At the beginning, $\mathcal{F}=\emptyset$. While $w\left(T_{r}^{*}\right)>3\lceil 1 / \alpha\rceil$, we perform the following iteration:

-Let $v$ be the lowest vertex in the tree $T^{*}$ such that $w\left(T_{v}^{*}\right)>\lceil 1 / \alpha\rceil$.

-If $w\left(T_{v}^{*}\right) \leq 2\lceil 1 / \alpha\rceil$, then we add the tree $T_{v}^{*}$ to $\mathcal{F}$, set $\lambda_{T_{v}^{*}}=V\left(T_{v}^{*}\right) \cap \mathcal{T}$, and delete all vertices and edges of $T_{v}^{*}$ from the tree $T^{*}$.

-Otherwise, let $u_{1}, \ldots, u_{k}$ be the children of $v$, and let $j$ be the smallest index such that $\sum_{i=1}^{j} w\left(T_{u_{i}}^{*}\right) \geq\lceil 1 / \alpha\rceil$. We add a new tree $T^{\prime}$ to $\mathcal{F}-$ a subtree of $T^{*}$ induced by $\{v\} \cup\left(\bigcup_{i=1}^{j} V\left(T_{u^{\prime}}^{*}\right)\right)$, setting $\lambda_{T^{\prime}}=\bigcup_{i=1}^{j}\left(V\left(T_{u_{i}}^{*}\right) \cap \mathcal{T}\right)$. We delete all edges of $T^{\prime}$ and all vertices of $V\left(T^{\prime}\right) \backslash\{v\}$ from the tree $T^{*}$.

Notice that since at the beginning of the current iteration $w\left(T_{r}^{*}\right)>3\lceil 1 / \alpha\rceil$, at the end of the current iteration, $w\left(T_{r}^{*}\right)>\lceil 1 / \alpha\rceil$ must hold. In the last iteration, when $w\left(T_{r}^{*}\right) \leq 3\lceil 1 / \alpha\rceil$, we add the tree $T_{r}^{*}$ to $\mathcal{F}$ and set $\lambda_{T_{r}^{*}}=V\left(T_{r}^{*}\right) \cap \mathcal{T}$. It is easy to verify that all conditions of the observation hold for the final collection $\mathcal{F}$ of trees.

Next we show that we can select at most one terminal from each set $\lambda_{T}$, for $T \in \mathcal{F}$, such that enough terminals from every subset $\mathcal{T}_{i}$ is selected.

Observation 2.13. There is an efficient algorithm that computes, for each $1 \leq i \leq \ell$, a subset $\mathcal{T}_{i}^{\prime} \subseteq \mathcal{T}_{i}$ of at least $\left\lfloor\frac{\kappa_{i}}{3\lceil 1 / \alpha\rceil}\right\rfloor$ vertices, so that, if we denote $\mathcal{T}^{\prime}=\bigcup_{i=1}^{\ell} \mathcal{T}_{i}^{\prime}$, then for every tree $T \in \mathcal{F},\left|\lambda_{T} \cap \mathcal{T}^{\prime}\right| \leq 1$.

From Claim 2.10, the resulting set $\mathcal{T}^{\prime}$ of terminals is $1 / 2$-well-linked in $G$. It now remains to prove Observation 2.13.

Proof. We build a node-capacitated directed flow network $\mathcal{N}$ as follows. We start from a source vertex $s$ and a destination vertex $s^{\prime}$ that have infinite capacity. We then add $\ell$ vertices $u_{1}, \ldots, u_{\ell}$, each of capacity $\kappa_{i}^{\prime}=\frac{\kappa_{i}}{3\lceil 1 / \alpha\rceil}$, and connect $s$ to each of these vertices. Each vertex $u_{i}$ will represent the set $\mathcal{T}_{i}$ of the terminals. For each terminal $t \in \mathcal{T}$, we add a unit-capacity vertex $v_{t}$ to $\mathcal{N}$, and if $t \in \mathcal{T}_{i}$, then we connect $u_{i}$ to $v_{t}$ with a directed edge.

For every tree $T \in \mathcal{F}$, we add a unit-capacity vertex $x_{T}$, which connects to the destination vertex $s^{\prime}$ with a directed edge. Finally, for every tree $T \in \mathcal{F}$, and for every 
terminal $t \in \lambda_{T}$, we add a directed edge $\left(v_{t}, x_{T}\right)$. We claim that there is a valid flow of value $\sum_{i=1}^{\ell} \kappa_{i}^{\prime}$ from $s$ to $s^{\prime}$ in $\mathcal{N}$. Indeed, consider a directed $s$-s $s^{\prime}$ path, and assume that the path is $\left(s, u_{i}, v_{t}, x_{T}, s^{\prime}\right)$. We send $\frac{1}{3\lceil 1 / \alpha\rceil}$ flow units along this path. Since for all $T \in \mathcal{F},\left|\lambda_{T}\right| \leq 3\lceil 1 / \alpha\rceil$, we obtain a valid $s$-s flow of value $\sum_{i=1}^{\ell} \kappa_{i}^{\prime}$. If we reduce the capacity of every vertex $u_{i}$, for $1 \leq i \leq \ell$, to $\left\lfloor\kappa_{i}^{\prime}\right\rfloor$, we can still obtain a valid $s-s^{\prime}$ flow of value $\sum_{i=1}^{\ell}\left\lfloor\kappa_{i}^{\prime}\right\rfloor$ by appropriately reducing flows on some paths. Since all vertex capacities are now integral, there is an integral flow $f$ of the same value. We are now ready to define the set $\mathcal{T}_{i}^{\prime}$ of terminals for each $1 \leq i \leq \ell$ : it contains all terminals $t \in \mathcal{T}_{i}$ such that the edge $\left(s_{i}, v_{t}\right)$ carries one flow unit in $f$. It is immediate to verify that $\left|\mathcal{T}_{i}^{\prime}\right|=\left\lfloor\kappa_{i}^{\prime}\right\rfloor \geq\left\lfloor\frac{\kappa_{i}}{3\lceil 1 / \alpha\rceil}\right\rfloor$, and since the capacities of all vertices $\left\{x_{T^{\prime}}\right\}_{T^{\prime} \in \mathcal{F}}$ are unit, if we denote by $\mathcal{T}^{\prime}=\bigcup_{i=1}^{\ell} \mathcal{T}_{i}^{\prime}$, then for every tree $T \in \mathcal{F},\left|\lambda_{T} \cap \mathcal{T}^{\prime}\right| \leq 1$.

The preceding claim gives a way to boost the well-linkedness of a given set $\mathcal{T}$ of terminals to 1/2-well-linkedness. This type of argument has been used before extensively, usually under the name of the "grouping technique" [Chekuri et al. 2013a, 2005; Rao and Zhou 2010; Andrews 2010; Chuzhoy 2016]. However, we need a stronger result: given a set $\mathcal{T}$ of terminals that are $\alpha$-well-linked in $G$, we would like to find a large subset $\mathcal{T}^{\prime} \subseteq \mathcal{T}$ such that $\mathcal{T}^{\prime}$ is node-well-linked in $G$. The following theorem allows us to achieve this, generalizing a similar theorem for edge-disjoint routing in Chekuri et al. [2013a]. The proof ${ }^{2}$ appears in the appendix.

TheOREM 2.14. Suppose that we are given a connected graph $G=(V, E)$ with maximum vertex degree at most $\Delta$, where $\Delta \geq 3$, and a subset $\mathcal{T}$ of $\kappa$ vertices called terminals, such that $\mathcal{T}$ is $\alpha$-well-linked in $G$, for some $0<\alpha \leq 1$. Then there is a subset $\mathcal{T}^{\prime} \subseteq \mathcal{T}$ of $\left\lceil\frac{3 \alpha \kappa}{10 \Delta}\right\rceil$ terminals, such that $\mathcal{T}^{\prime}$ is node-well-linked in $G$. Moreover, there is an algorithm whose running time is polynomial in $|V|$ and $\kappa$ that computes a subset $\mathcal{T}^{\prime} \subseteq \mathcal{T}$ of at least $\frac{\alpha}{32 \Delta^{4} \beta_{\mathrm{ARV}}(\kappa)} \cdot \kappa$ terminals, such that $\mathcal{T}^{\prime}$ is node-well-linked in $G$.

CoROLlary 2.15. There is an efficient algorithm that, given a connected graph $G=$ $(V, E)$ with maximum vertex degree $\Delta \geq 3$, a subset $\mathcal{T}$ of $\kappa$ vertices of $G$ that are $\alpha$-welllinked in $G$ for some $0<\alpha \leq 1$, and a partition $\mathcal{T}_{1}, \ldots, \mathcal{T}_{\ell}$ of $\mathcal{T}$ into disjoint subsets, computes, for each $1 \leq i \leq \ell$, a subset $\mathcal{T}_{i}^{\prime} \subseteq \mathcal{T}_{i}$ of $\left\lfloor\frac{\alpha \cdot\left|\mathcal{T}_{i}\right|}{2^{10} \Delta^{5} \beta_{\mathrm{ARV}}(\kappa)}\right\rfloor$ terminals, such that $(i)$ for all $1 \leq i \leq \ell$, set $\mathcal{T}_{i}^{\prime}$ is node-well-linked in $G$; (ii) $\bigcup_{i=1}^{\ell} \mathcal{T}_{i}^{\prime}$ is $1 / 2$-well-linked in $G\left[S_{i}\right]$; and (iii) for all $1 \leq i<j \leq \ell, \mathcal{T}_{i}^{\prime}$ and $\mathcal{T}_{j}^{\prime}$ are linked in $G$.

Proof. For all $1 \leq i \leq \ell$, let $\kappa_{i}=\left|\mathcal{T}_{i}\right|$. We use Corollary 2.11 to compute, for each $1 \leq i \leq \ell$, a subset $\mathcal{T}_{i}^{1} \subseteq \mathcal{T}_{i}$ of at least $\left\lfloor\frac{\kappa_{i}}{3\lceil 1 / \alpha\rceil}\right\rfloor \geq\left\lfloor\frac{\alpha \kappa_{i}}{8}\right\rfloor$ terminals such that $\bigcup_{i=1}^{\ell} \mathcal{T}_{i}^{1}$ is $1 / 2$-well-linked in $G$.

Next, for each $1 \leq i \leq \ell$, we apply Theorem 2.14 to $\mathcal{T}_{i}^{1}$, to compute a subset $\mathcal{T}_{i}^{2} \subseteq \mathcal{T}_{i}^{1}$ of at least $\frac{\left|\mathcal{T}_{i}^{1}\right|}{32 \Delta^{4} \beta_{\mathrm{ARV}}\left(\kappa_{i}\right)} \geq \frac{\alpha \kappa_{i}}{256 \Delta^{4} \beta_{\mathrm{ARV}}(\kappa)}$ terminals so that $\mathcal{T}_{i}^{2}$ is node-well-linked in $G$. We then let $\mathcal{T}_{i}^{\prime} \subseteq \mathcal{T}_{i}^{2}$ be a subset of $\left\lfloor\frac{\left|\mathcal{T}_{i}^{2}\right|}{4 \Delta}\right\rfloor \geq\left\lfloor\frac{\alpha \kappa_{i}}{2^{10} \Delta^{5} \beta_{\operatorname{ARV}}(\kappa)}\right\rfloor$ terminals. Since the terminals of $\bigcup_{j=1}^{\ell} \mathcal{T}_{j}^{1}$ are $1 / 2$-well-linked in $G$, from Theorem 2.9 , for all $1 \leq j<j^{\prime} \leq \ell, \mathcal{T}_{j}^{\prime}$ and $\mathcal{T}_{j^{\prime}}^{\prime}$ are linked in $G$.

\footnotetext{
${ }^{2}$ Some of our theorems on well-linked sets, including this one, appear to have alternate proofs via tangles and related matroids from graph minor theory [Robertson and Seymour 1991]; this was suggested to us by a reviewer. However, it is unclear whether the alternate proofs yield polynomial-time algorithms.
} 


\subsection{Treewidth and Well-Linkedness}

The following lemma summarizes an important connection between the graph treewidth and the size of the largest node-well-linked set of vertices.

LEMMA 2.16 [REED 2007]. Let $k$ be the size of the largest node-well-linked vertex set in G. Then, $\frac{k}{4}-1 \leq \operatorname{tw}(G) \leq k-1$.

Lemma 2.16 guarantees that a graph $G$ of treewidth $k$ contains a set $X$ of $\Omega(k)$ vertices that is node-well-linked in $G$. Kreutzer and Tazari [2010] give a constructive version of this lemma, obtaining a set $X$ with slightly weaker properties. Lemma 2.17 rephrases, in terms convenient to us, Lemma 3.7 in Kreutzer and Tazari [2010]. ${ }^{3}$

LEMma 2.17. There is an efficient algorithm that, given a graph $G$ of treewidth $k$, finds a set $X$ of $\Omega(k)$ vertices, such that $X$ is $\alpha^{*}=\Omega(1 / \log k)$-well-linked in $G$ and $|X|$ is even. Moreover, for every partition $\left(X_{1}, X_{2}\right)$ of $X$ into two equal-size subsets, there is a collection $\mathcal{P}$ of paths connecting every vertex of $X_{1}$ to a distinct vertex of $X_{2}$, such that every vertex of $G$ participates in at most $1 / \alpha^{*}$ paths in $\mathcal{P}$.

\subsection{A Tree With Many Leaves or a Long 2-Path}

Suppose that we are given a connected $n$-vertex graph $Z$. A path $P$ in $Z$ is called a 2 -path if every vertex $v \in P$ has degree 2 in $Z$. The following theorem, due to Leaf and Seymour [2015], states that we can find either a spanning tree with many leaves or a long 2-path in $Z$. For completeness, the proof appears in the appendix.

THEOREM 2.18. There is an efficient algorithm that, given a connected n-vertex graph $Z$, and integers $L \geq 1, p \geq 1$ with $\frac{n}{2 L} \geq p+5$, either finds a spanning tree $T$ with at least $L$ leaves in $Z$ or a 2-path containing at least $p$ vertices in $Z$.

\subsection{Rerouting Two Sets of Disjoint Paths}

Suppose that we are given a directed graph $\hat{G}$, a set $U \subseteq V(\hat{G})$ of its vertices, and an additional vertex $s \in V(\hat{G}) \backslash U$. A set $\mathcal{X}$ of directed paths that originate at the vertices of $U$ and terminate at $s$ is called a set of $U$-s paths. We say that the paths in $\mathcal{X}$ are nearly disjoint if except for vertex $s$ they do not share other vertices. We need the following lemma, which was proved by Conforti et al. [2003]. We provide a simpler proof, suggested to us by Seymour (pers. comm.) in the appendix.

LEMma 2.19. There is an efficient algorithm that, given a directed graph $\hat{G}$, two subsets $U_{1}, U_{2}$ of its vertices, and an additional vertex $s \in V(\hat{G}) \backslash\left(U_{1} \cup U_{2}\right)$, together with a set $\mathcal{X}_{1}$ of $\ell_{1}$ nearly disjoint $U_{1}$-s paths and a set $\mathcal{X}_{2}$ of $\ell_{2}$ nearly disjoint $U_{2}-s$ paths in $\hat{G}$, where $\ell_{1}>\ell_{2} \geq 1$, finds a set $\mathcal{X}^{\prime}$ of $\ell_{1}$ nearly disjoint $\left(U_{1} \cup U_{2}\right)$-s paths, and a partition $\left(\mathcal{X}_{1}^{\prime}, \mathcal{X}_{2}^{\prime}\right)$ of $\mathcal{X}^{\prime}$, such that $\left|\mathcal{X}_{2}^{\prime}\right|=\ell_{2}$, the paths of $\mathcal{X}_{2}^{\prime}$ originate from $U_{2}$, and $\mathcal{X}_{1}^{\prime} \subseteq \mathcal{X}_{1}$

\subsection{Cut-Matching Game and Degree Reduction}

We say that a graph $G=(V, E)$ is an $\alpha$-expander if $\min _{\substack{0<|S| \leq|V| \\ S \subseteq}}\left\{\frac{|E(S, \bar{S})|}{|S|}\right\} \geq \alpha$. Equivalently, $G$ is an $\alpha$-expander if $\Phi_{V}(G) \geq \alpha$.

We use the cut-matching game of Khandekar et al. [2009]. In this game, we are given a set $V$ of $N$ vertices, where $N$ is even, and two players: a cut player, whose goal is to construct an expander $X$ on the set $V$ of vertices, and a matching player, whose

\footnotetext{
${ }^{3}$ Lemma 2.17 is slightly weaker than what was shown in Kreutzer and Tazari [2010]. We use it since it suffices for our purposes and avoids the introduction of additional notation.
} 
goal is to delay its construction. The game is played in iterations. We start with the graph $X$ containing the set $V$ of vertices, and no edges. In each iteration $j$, the cut player computes a bipartition $\left(A_{j}, B_{j}\right)$ of $V$ into two equal-size sets, and the matching player returns some perfect matching $M_{j}$ between the two sets. The edges of $M_{j}$ are then added to $X$. Khandekar et al. have shown that there is a strategy for the cut player, guaranteeing that after $O\left(\log ^{2} N\right)$ iterations we obtain a $\frac{1}{2}$-expander with high probability. Subsequently, Orecchia et al. [2008] have shown the following improved bound.

Theorem 2.20 [ORECCHIA ET AL. 2008]. There is a randomized algorithm for the cut player, such that, no matter how the matching player plays, after $\gamma_{\mathrm{CMG}}(N)=O\left(\log ^{2} N\right)$ iterations, graph $X$ is an $\alpha_{\mathrm{CMG}}(N)=\Omega(\log N)$-expander, with constant probability.

\subsection{Starting Point}

Let $G$ be a graph with $\operatorname{tw}(G)=k$. The proof of Theorem 1.1 uses the notion of edge-welllinkedness as well as node-well-linkedness. To be able to translate between both types of well-linkedness and the treewidth, we need to reduce the maximum vertex degree of the input graph $G$. Using the cut-matching game, one can reduce the maximum vertex degree to $O\left(\log ^{3} k\right)$ while only losing a poly $\log k$ factor in the treewidth, as was noted in Chekuri and Ene [2013] (see Remark 2.2). The following theorem, whose proof appears in the appendix, provides the starting point for our algorithm.

THEOREM 2.21. There is an efficient randomized algorithm that, given a graph $G$ with $\operatorname{tw}(G)=k$, computes a subgraph $G^{\prime}$ of $G$ with maximum vertex degree $\Delta=O\left(\log ^{3} k\right)$, and a subset $Z$ of $\Omega(k /$ poly $\log k)$ vertices of $G^{\prime}$, such that $Z$ is node-well-linked in $G^{\prime}$, with high probability.

We note that one can also reduce the degree to a constant with an additional polylog(k) factor loss in the treewidth [Chekuri and Ene 2013]; however, that result also relies on the preceding theorem as a starting point. The constant can be made 4 with a polynomial factor loss in treewidth [Kreutzer and Tazari 2010], which we would not wish to lose. We also note that Chekuri and Chuzhoy [2015] have shown that the degree can be reduced to 3 , and a set $X$ of cardinality $\Omega(k /$ poly $\log k)$ as in the theorem can be computed efficiently, but that proof builds on the present work.

\section{A PATH-OF-SETS SYSTEM}

In this section, we define our main combinatorial object, called a path-of-sets system. We start with a few definitions.

Suppose that we are given a collection $\mathcal{S}=\left\{S_{1}, \ldots, S_{\ell}\right\}$ of disjoint vertex subsets of $V(G)$. Let $S_{i}, S_{j} \in \mathcal{S}$ be two such subsets. We say that a path $P=\left(v_{1}, \ldots, v_{h}\right)$ connects $S_{i}$ to $S_{j}$ if and only if the first vertex $v_{1}$ belongs to $S_{i}$ and the last vertex $v_{h}$ belongs to $S_{j}$. We say that $P$ connects $S_{i}$ to $S_{j}$ directly if, additionally, $P$ does not contain vertices of $\bigcup_{S \in \mathcal{S}} S$ as inner vertices.

Definition 3.1. A path-of-sets system of width $w$ and length $\ell$ consists of

-a sequence ${ }^{4} \mathcal{S}=\left(S_{1}, \ldots, S_{\ell}\right)$ of $\ell$ disjoint vertex subsets of $G$, where for each $i, G\left[S_{i}\right]$ is connected;

-for each $1 \leq i \leq \ell$, two disjoint sets $A_{i}, B_{i} \subseteq S_{i}$ of vertices of cardinality $w$ each such that sets $A_{i}$ and $B_{i}$ are linked in $G\left[S_{i}\right]$; and

\footnotetext{
${ }^{4}$ We also interpret $\mathcal{S}$ as a collection of sets for notational ease.
} 
-for each $1 \leq i<\ell$, a set $\mathcal{P}_{i}$ of $w$ disjoint paths, connecting the vertices of $B_{i}$ to the vertices of $A_{i+1}$ directly (i.e., paths in $\mathcal{P}_{i}$ do not contain the vertices of $\bigcup_{S \in \mathcal{S}} S$ as inner vertices), such that all paths in $\bigcup_{i} \mathcal{P}_{i}$ are mutually disjoint. (See Figure 1.)

We say that it is a strong path-of-sets system if additionally for each $1 \leq i \leq \ell, A_{i}$ is node-well-linked in $G\left[S_{i}\right]$, and so is $B_{i}$.

Notice that a path-of-sets system is completely determined by the sequence $\mathcal{S}$ of vertex subsets; the collection $\bigcup_{i=1}^{\ell-1} \mathcal{P}_{i}$ of paths; and the sets $A_{1} \subseteq S_{1}, B_{\ell} \subseteq S_{\ell}$ of vertices. In the following, we denote path-of-sets systems by $\left(\mathcal{S}, \bigcup_{i} \mathcal{P}_{i}, A_{1}, B_{\ell}\right)$.

We note that Leaf and Seymour [2015] have defined a very similar object, referred to as a $(w, \ell)$-grill, and they showed that the two objects are roughly equivalent. Namely, a path-of-sets system with parameters $w$ and $\ell$ contains a $(w, \ell)$-grill as a minor, whereas a $(w, \ell)$-grill contains a path-of-sets system of width $w$ and length $\Omega(\ell / w)$. They also show an efficient algorithm that, given a $(w, \ell)$-grill with $w=\Omega\left(g^{3}\right)$ and $\ell=\Omega\left(g^{4}\right)$, finds a model of the $(g \times g)$-grid minor in the grill. ${ }^{5}$

Our goal is to show that a graph containing a large enough path-of-sets system must also contain a large grid minor. The following theorem is a starting point. The proof appears in the appendix.

THEOREm 3.2. There is an efficient algorithm that, given a connected graph $G=(V, E)$, two disjoint subsets $A, B \subseteq V$ of its vertices with $|A|=|B|=w$, such that $A, B$ are linked in $G$, and integers $h_{1}, h_{2}>1$ with $\left(16 h_{1}+10\right) h_{2} \leq w$, either returns a model of the $\left(h_{1} \times h_{1}\right)$-grid minor in $G$ or computes a collection $\mathcal{P}$ of $h_{2}$ node-disjoint paths, connecting vertices of $A$ to vertices of $B$, such that for every pair $P, P^{\prime} \in \mathcal{P}$ of paths with $P \neq P^{\prime}$, there is a path $\beta_{P, P^{\prime}} \subseteq G$, connecting a vertex of $P$ to a vertex of $P^{\prime}$, where $\beta_{P, P^{\prime}}$ is internally disjoint from $\bigcup_{P^{\prime \prime} \in \mathcal{P}} V\left(P^{\prime \prime}\right)$.

Given a path-of-sets system $\left(\mathcal{S}, \bigcup_{i=1}^{\ell-1} \mathcal{P}_{i}, A_{1}, B_{\ell}\right)$ in $G$, we say that $G^{\prime}$ is a subgraph of $G$ spanned by the path-of-sets system if $G^{\prime}$ is the union of $G\left[S_{i}\right]$ for all $1 \leq i \leq \ell$ and all paths in $\bigcup_{i=1}^{\ell-1} \mathcal{P}_{i}$.

The following corollary of Theorem 3.2 allows us to obtain a grid minor from a pathof-sets system. Its proof appears in the appendix.

COROLlary 3.3. There is an efficient algorithm that, given a graph $G$, a path-of-sets system $\left(\mathcal{S}, \bigcup_{i=1}^{\ell-1} \mathcal{P}_{i}, A_{1}, B_{\ell}\right)$ of length $\ell \geq 2$ and width $w$ in $G$, and integers $h_{1}, h_{2}$ with $\left(16 h_{1}+10\right) h_{2} \leq w$, either returns a model of the $\left(h_{1} \times h_{1}\right)$-grid minor in the subgraph $G^{\prime}$ of $G$ spanned by the path-of-sets system or returns a collection $\mathcal{Q}$ of $h_{2}$ node-disjoint paths in $G^{\prime}$, connecting vertices of $A_{1}$ to vertices of $B_{\ell}$, such that for all $1 \leq i \leq \ell$, for every path $Q \in \mathcal{Q}, S_{i} \cap Q$ is a path, and $S_{1} \cap Q, S_{2} \cap Q, \ldots, S_{\ell} \cap Q$ appear on $Q$ in this order. Moreover, for every $1 \leq j \leq\lfloor\ell / 2\rfloor$, for every pair $Q, Q^{\prime} \in \mathcal{Q}$ of paths, there is a path $\beta_{2 i}\left(Q, Q^{\prime}\right) \subseteq G^{\prime}\left[S_{2 i}\right]$, connecting a vertex of $Q$ to a vertex of $Q^{\prime}$, such that $\beta_{2 i}\left(Q, Q^{\prime}\right)$ is internally disjoint from all paths in $\mathcal{Q}$.

The following corollary completes the construction of the grid minor, slightly improving on a similar result of Leaf and Seymour [2015]. The proof is included in the appendix.

\footnotetext{
${ }^{5}$ In fact, Leaf and Seymour [2015] show a slightly stronger result that a $(w, \ell)$-grill with $w \geq(2 g+1)(2 r-5)+2$ and $\ell \geq r(2 g+r-2)$ contains a $(g \times g)$-grid minor or a bipartite-clique $K_{r, r}$ as a minor. This can give slightly improved bounds on the grid minor size if the given graph excludes bipartite-clique minors for small $r$.
} 
Corollary 3.4. There is an efficient algorithm that, given a graph $G$, an integer $g>1$ and a path-of-sets system $\left(\mathcal{S}, \bigcup_{i=1}^{\ell-1} \mathcal{P}_{i}, A_{1}, B_{\ell}\right)$ of width $w=16 g^{2}+10 g$ and length $\ell=2 g(g-1)$ in $G$, computes a model of the $(g \times g)$-grid minor in $G$.

The main technical contribution of our article is summarized in the following theorem.

THEOREM 3.5. There are constants $c^{\prime}, c^{\prime \prime}>0$ and an efficient randomized algorithm that, given a graph $G$ of treewidth $k$ and integral parameters $w^{*}, \ell^{*}>2$, such that $k / \log ^{c^{\prime}} k>c^{\prime \prime} w^{*}\left(\ell^{*}\right)^{48}$, with high probability returns a strong path-of-sets system of width $w^{*}$ and length $\ell^{*}$ in $G$.

Choosing $w^{*}, \ell^{*}=\Omega\left(k^{1 / 49} /\right.$ poly $\left.\log k\right)$, from Theorem 3.5, we can efficiently construct a path-of-sets system of width $w^{*}$ and length $\ell^{*}$ in $G$ with high probability. From Corollary 3.4, we can then efficiently construct a model of a grid minor of size $\left(\Omega\left(k^{1 / 98} /\right.\right.$ poly $\left.\log k\right) \times \Omega\left(k^{1 / 98} /\right.$ poly $\left.\left.\log k\right)\right)$. The rest of this article is mostly dedicated to proving Theorem 3.5. In Section 6, we provide some extensions to this theorem that we believe may be useful in various applications, such as algorithms for routing problems.

\section{CONSTRUCTING A PATH-OF-SETS SYSTEM}

We can view a path-of-sets system as a meta-path, whose vertices $v_{1}, \ldots, v_{\ell}$ correspond to the sets $S_{1}, \ldots, S_{\ell}$, and each edge $e=\left(v_{i}, v_{i+1}\right)$ corresponds to the collection $\mathcal{P}_{i}$ of $w$ disjoint paths. Unfortunately, we do not know how to find such a meta-path directly (except for $\ell=O(\log k)$, which is not enough for us). As we show in the following, a generalization of the work of Chuzhoy and Li [2016], combined with some ideas from Chekuri and Ene [2013], gives a construction of a meta-tree of degree at most 3 instead of the meta-path. We define the corresponding object, which we call a tree-of-sets system. We start with the following definitions.

Definition 4.1. Given a set $S$ of vertices in graph $G$, the interface of $S$ is $\Gamma_{G}(S)=$ $\left\{v \in S \mid \exists e=(u, v) \in \operatorname{out}_{G}(S)\right\}$. We say that $S$ has the $\alpha$-bandwidth property in $G$ if its interface $\Gamma_{G}(S)$ is $\alpha$-well-linked in $G[S]$.

Definition 4.2. A tree-of-sets system with parameters $\ell, w, \alpha_{\mathrm{BW}}(\ell, w \geq 1$ are integers and $0<\alpha_{\mathrm{BW}}<1$ is real-valued) consists of

-a collection $\mathcal{S}=\left\{S_{1}, \ldots, S_{\ell}\right\}$ of $\ell$ disjoint vertex subsets of $G$, where for each $1 \leq i \leq \ell$, $G\left[S_{i}\right]$ is connected;

-a tree $T$ with $V(T)=\left\{v_{1}, \ldots, v_{\ell}\right\}$, whose maximum vertex degree is at most 3 ;

-for each edge $e=\left(v_{i}, v_{j}\right)$ of $T$, a set $\mathcal{P}_{e}$ of $w$ disjoint paths, connecting $S_{i}$ to $S_{j}$ directly (i.e., paths in $\mathcal{P}_{e}$ do not contain the vertices of $\bigcup_{S \in \mathcal{S}} S$ as inner vertices).

Moreover, all paths in $\mathcal{P}=\bigcup_{e \in E(T)} \mathcal{P}_{e}$ are pairwise disjoint

and have the following additional property. Let $G^{\prime}$ be the subgraph of $G$ obtained by the union of $G\left[S_{i}\right]$ for all $S_{i} \in \mathcal{S}$ and $\bigcup_{e \in E(T)} \mathcal{P}(e)$. Then each $S_{i} \in \mathcal{S}$ has the $\alpha_{\mathrm{BW}}$-bandwidth property in $G^{\prime}$.

We say that the graph $G^{\prime}$ defined earlier is a subgraph of $G$ spanned by the tree-of-sets system.

We remark that a tree-of-sets system is closely related to the path-of-sets system: a path-of-sets system is a tree-of-sets system where the tree is restricted to be a path; it is easy to verify that the linkedness property of sets $A_{i}, B_{i}$ inside every cluster $S_{i}$ guarantee the 1/4-bandwidth property of $S_{i}$ in $G^{\prime}$.

The following theorem describes our construction of a tree-of-sets system. It strengthens the results of Chuzhoy and Li [2016], and its proof appears in Section 5. 
THEOREM 4.3. There is a constant $c$ and an efficient randomized algorithm that takes as input (i) a graph $G$ of maximum degree $\Delta$; (ii) a subset $\mathcal{T}$ of $k$ vertices in $G$ called terminals, such that $\mathcal{T}$ is node-well-linked in $G$ and the degree of every vertex in $\mathcal{T}$ is 1; and (iii) two integer parameters $\ell>1, w>4 \log k$, such that $k / \log ^{4} k>c w \ell^{19} \Delta^{8}$, and with high probability outputs a tree-of-sets system $\left(\mathcal{S}, T, \bigcup_{e \in E(T)} \mathcal{P}_{e}\right)$ in $G$, with parameters $w, \ell$ and $\alpha_{\mathrm{BW}}=\Omega\left(\frac{1}{\ell^{2} \log ^{1.5} k}\right)$. Moreover, for all $S_{i} \in \mathcal{S}, S_{i} \cap \mathcal{T}=\emptyset$.

We prove Theorem 4.3 in the following section and show how to construct a path-ofsets system using this theorem here.

Suppose that we are given a tree-of-sets system $\left(\mathcal{S}, T, \bigcup_{e \in E(T)} \mathcal{P}(e)\right)$, and an edge $e \in E(T)$, incident on a vertex $v_{i} \in V(T)$. We denote by $\delta_{S_{i}}(e) \subseteq S_{i}$ the set of all vertices of $S_{i}$ that serve as endpoints of paths in $\mathcal{P}(e)$.

Definition 4.4. A tree-of-sets system $\left(\mathcal{S}, T, \bigcup_{e \in E(T)} \mathcal{P}(e)\right)$ with parameters $w, \ell, \alpha_{\mathrm{BW}}$ is a strong tree-of-sets system, if and only if for each $S_{i} \in \mathcal{S}$,

—for each edge $e \in E(T)$ incident to $v_{i}$, the set $\delta_{S_{i}}(e) \subseteq S_{i}$ of vertices is node well-linked in $G\left[S_{i}\right]$; and

—for every pair $e, e^{\prime} \in E(T)$ of edges incident to $v_{i}$, the sets $\delta_{S_{i}}(e), \delta_{S_{i}}\left(e^{\prime}\right) \subseteq S_{i}$ of vertices are linked in $G\left[S_{i}\right]$

The following lemma allows us to transform an arbitrary tree-of-sets system into a strong one.

LEMMA 4.5. There is an efficient algorithm that, given a graph $G$ with maximum vertex degree at most $\Delta$ and a tree-of-sets system $\left(\mathcal{S}, T, \bigcup_{e \in E(T)} \mathcal{P}_{e}\right)$ with parameters $\ell, w, \alpha_{\mathrm{BW}}$ in $G$, outputs a strong tree-of-sets system $\left(\mathcal{S}, T, \bigcup_{e \in E(T)} \mathcal{P}_{e}^{*}\right)$ with parameters $\ell, \tilde{w}, \frac{1}{2}$ such that for each $e \in E(T) \mathcal{P}_{e}^{*} \subseteq \mathcal{P}_{e}$, and $\tilde{w}=\Omega\left(\frac{\alpha_{\mathrm{BW}}^{2}}{\Delta^{10}\left(\beta_{\mathrm{ARV}}(w)\right)^{2}} \cdot w\right)$.

Proof. We assume that tree $T$ is rooted at some vertex whose degree is greater than 1 . We process the vertices of the tree $T$ in the bottom-up fashion: in other words, we only process a vertex $v_{i}$ after all of its descendants have been processed. Assume first that $v_{i}$ is a leaf vertex, and let $e$ be the unique edge incident to $v$ in $T$. We use Corollary 2.15 to compute a subset $\delta^{\prime} \subseteq \delta_{S_{i}}(e)$ of at least $\left\lfloor\frac{\alpha_{\mathrm{BW}} w}{2^{11} \Delta^{5} \beta_{\mathrm{ARV}}(w)}\right\rfloor$ vertices such that the vertices of $\delta^{\prime}$ are node-well-linked in $G\left[S_{i}\right]$. We then discard from $\mathcal{P}(e)$ all paths except those whose endpoint lies in $\delta^{\prime}$.

Consider now some nonleaf vertex $v_{i}$ of the tree, and assume that it has degree 3 (the case where $v_{i}$ has degree 2 is dealt with similarly). Let $e_{1}, e_{2}, e_{3}$ be the edges incident to $v_{i}$ in $T$, and for each $1 \leq j \leq 3$, let $\delta_{j}=\delta_{S_{i}}\left(e_{j}\right)$; note that $\delta_{j}$ is based on the current set of paths $\mathcal{P}(e)$ as we process the tree. Recall that the set $\delta_{1} \cup \delta_{2} \cup \delta_{3}$ of vertices is $\alpha_{\mathrm{BW}}$-well-linked in $G\left[S_{i}\right]$. We use Corollary 2.15 to compute, for each $1 \leq j \leq 3$, a subset $\delta_{j}^{\prime}$ of at least $\left\lfloor\frac{\alpha_{\mathrm{BW}}\left|\delta_{j}\right|}{2^{11} \Delta^{5} \beta_{\mathrm{ARV}}(w)}\right\rfloor$ vertices so that each of the sets $\delta_{j}^{\prime}$ is node-well-linked in $G\left[S_{i}\right]$, every pair of such sets is linked in $G\left[S_{i}\right]$, and $\delta_{1}^{\prime} \cup \delta_{2}^{\prime} \cup \delta_{3}^{\prime}$ is $1 / 2$-well-linked in $G\left[S_{i}\right]$. For $1 \leq j \leq 3$, we discard paths from $\mathcal{P}\left(e_{j}\right)$ that do not have an endpoint in $\delta_{j}^{\prime}$. Once all vertices of $T$ are processed, we claim that for every edge $e \in E(T)$, the resulting set $\mathcal{P}(e)$ contains at least $\Omega\left(\frac{\alpha_{\mathrm{BW}}^{2}}{\Delta^{10}\left(\beta_{\mathrm{ARV}}(w)\right)^{2}} \cdot w\right)$ paths, and the new tree-of-sets system is guaranteed to be strong. The latter property is easy to see. For the former, consider an edge $e=\left(v_{i}, v_{i^{\prime}}\right) \in E(T)$, where $v_{i^{\prime}}$ is the parent of $v_{i}$. Before processing $v_{i}$, 
there are $w$ paths in $\mathcal{P}(e)$. After processing $v_{i}$, there are least $\delta^{\prime}=\left\lfloor\frac{\alpha_{\mathrm{BW}} w}{2^{11} \Delta^{5} \beta_{\mathrm{ARV}}(w)}\right\rfloor$ paths left in $\mathcal{P}(e)$. After processing $v_{i^{\prime}}$, there are at least $\delta^{\prime \prime}=\left\lfloor\frac{\alpha_{\mathrm{BW}}\left|\delta^{\prime}\right|}{2^{11} \Delta^{5} \beta_{\mathrm{ARV}}(w)}\right\rfloor$ paths that remain in $\mathcal{P}(e)$. Paths in $\mathcal{P}(e)$ are only eliminated when processing $v_{i}$ and $v_{i^{\prime}}$, and this gives us the desired claim.

The following theorem allows us to obtain a strong path-of-sets system from a strong tree-of-sets system.

THEOREM 4.6. There is an efficient algorithm that, given a graph $G$ and a strong tree-of-sets system $\left(\mathcal{S}, T, \bigcup_{e \in E(T)} \mathcal{P}_{e}^{*}\right)$ with parameters $\ell, \tilde{w}, \frac{1}{2}$, and integers $w^{*}, \ell^{*}>1$, such that $\left(\ell^{*}\right)^{2} \leq \ell$ and $\tilde{w}>16 w^{*}\left(\ell^{*}\right)^{2}+1$, outputs a strong path-of-sets system $\left(\mathcal{S}^{\prime}, \bigcup_{i=1}^{\ell^{*}-1} \mathcal{P}_{i}, A_{1}, B_{\ell^{*}}\right)$ of length $\ell^{*}$ and width $w^{*}$, with $\mathcal{S}^{\prime} \subseteq \mathcal{S}$.

Before we prove the preceding theorem, we use the results stated so far to complete the proof of Theorem 3.5.

Proof of Theorem 3.5. We assume that $k$ is large enough, so, for example, $k^{1 / 30}>$ $c^{*} \log k$ for some large enough constant $c^{*}$. Given a graph $G=(V, E)$ with treewidth $k$, we use Theorem 2.21 to compute a subgraph $G^{\prime}$ of $G$ with maximum vertex degree $\Delta=O\left(\log ^{3} k\right)$, and a set $X$ of $\Omega(k /$ poly $\log k)$ vertices, such that $X$ is node-well-linked in $G^{\prime}$. We add a new set $\mathcal{T}$ of $|X|$ vertices, each of which connects to a distinct vertex of $X$ with an edge. For convenience, we denote this new graph by $G$, and $|\mathcal{T}|$ by $k$, and we refer to the vertices of $\mathcal{T}$ as terminals. Clearly, the maximum vertex degree of $G$ is at most $\Delta=O\left(\log ^{3} k\right)$, the degree of every terminal is 1 , and $\mathcal{T}$ is node-well-linked in $G$. We can now assume that $\frac{k}{\Delta^{19} \log ^{8} k}>\hat{c} w^{*}\left(\ell^{*}\right)^{48}$ for some large enough constant $\hat{c}$.

We set $\ell=\left(\ell^{*}\right)^{2}$ and $w=\frac{\hat{c}}{c} \cdot w^{*}\left(\ell^{*}\right)^{10} \Delta^{11} \log ^{4} k$, so $w>4 \log k$ holds, where $c$ is the constant from Theorem 4.3. Clearly,

$$
c w \ell^{19} \Delta^{8}=\left(\hat{c} w^{*}\left(\ell^{*}\right)^{10} \Delta^{11} \log ^{4} k\right) \cdot\left(\ell^{*}\right)^{38} \Delta^{8}=\hat{c} w^{*}\left(\ell^{*}\right)^{48} \Delta^{19} \log ^{4} k .
$$

Therefore, $\frac{k}{\log ^{4} k}>c w \ell^{19} \Delta^{8}$. We then apply Theorem 4.3 to $G$ and $\mathcal{T}$ to obtain a tree-of-sets system $\left(\mathcal{S}, T, \bigcup_{e \in E(T)} \mathcal{P}_{e}\right)$, with parameters $\ell, w$ and $\alpha_{\mathrm{BW}}=\Omega\left(\frac{1}{\ell^{2} \log ^{1.5} k}\right)$.

We use Lemma 4.5 to convert $\left(\mathcal{S}, T, \bigcup_{e \in E(T)} \mathcal{P}_{e}\right)$ into a strong tree-of-set system $\left(\mathcal{S}, T, \bigcup_{e \in E(T)} \mathcal{P}_{e}^{*}\right)$ with parameters $\ell$ and $\tilde{w}=\Omega\left(\frac{\alpha_{\mathrm{BW}}^{2}}{\Delta^{10}\left(\beta_{\mathrm{ARV}}(w)\right)^{2}} \cdot w\right)$. If $\hat{c}$ is chosen to be large enough, $\tilde{w}>16 w^{*}\left(\ell^{*}\right)^{2}+1$ must hold. We then apply Theorem 4.6 to obtain a path-of-set system with width $w^{*}$ and length $\ell^{*}$.

We now prove Theorem 4.6.

Proof of Theorem 4.6. Let $\left(\mathcal{S}, T, \bigcup_{e \in E(T)} \mathcal{P}_{e}^{*}\right)$ be the tree-of-set system with parameters $\ell$ and $\tilde{w}$. For convenience, for each set $S \in \mathcal{S}$, we denote the corresponding vertex of tree $T$ by $v_{S}$. If tree $T$ contains a root-to-leaf path of length at least $\ell^{*}$, then we are done, as this path gives a path-of-sets system of width $\tilde{w} \geq w^{*}$ and length $\ell^{*}$. The path-of-sets system is strong, since for every edge $e=\left(v_{i}, v_{i^{\prime}}\right) \in E(T), \delta_{S_{i}}(e)$ is node-well-linked in $G\left[S_{i}\right]$.

Otherwise, since $|V(T)|=\ell \geq\left(\ell^{*}\right)^{2}, T$ must contain at least $\ell^{*}+1$ leaves (see Claim 2.2). Let $L$ be a subset of $\ell^{*}$ leaves of $T$, and let $\mathcal{L} \subseteq \mathcal{S}$ be the collection of $\ell^{*}$ clusters, whose corresponding vertices belong to $L$, so $\mathcal{L}=\left\{S \in \mathcal{S} \mid v_{S} \in L\right\}$. We next show how to build a path-of-sets system $\left(\mathcal{S}^{\prime}, \bigcup_{i=1}^{\ell^{*}-1} \mathcal{P}_{i}, A_{1}, B_{\ell^{*}}\right)$, whose collection of clusters is $\mathcal{S}^{\prime}=\mathcal{L}$. 


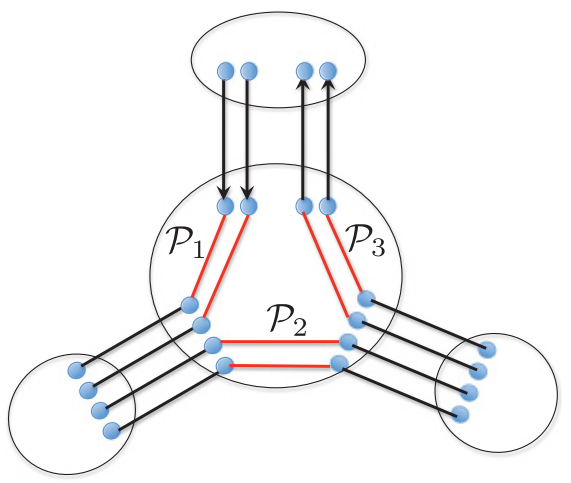

Fig. 2. Routing paths of the DFS tour inside $S$.

Intuitively, we would like to perform a depth-first-search (DFS) tour on our meta-tree $T$. This should be done with many paths in parallel. In other words, we want to build $w^{*}$ disjoint paths that visit the clusters in $\mathcal{S}$ in the same order-the order of the tour. The clusters in $\mathcal{L}$ will then serve as the sets $\mathcal{S}^{\prime}$ in our final path-of-sets system, and the collection of $w^{*}$ paths that we build will be used for the path sets $\mathcal{P}_{i}$. For this to work, we need to route up to three sets of paths across clusters $S \in \mathcal{S}$. For example, if the vertex $v_{S}$ corresponding to the cluster $S$ is a degree-3 vertex in $T$, then for the DFS tour, we need to route three sets of paths across $S$ : one set connecting the paths coming from the parent of $v_{S}$ to its first child, one set connecting the paths coming back from the first child to the second child, and one set connecting the paths coming back from the second child to the parent of $v_{S}$ (Figure 2). Even though every pair of relevant vertex subsets on the interface of $S$ is linked, this property only guarantees that we can route one such set of paths, which presents a major technical difficulty in using this approach directly.

Our algorithm consists of two phases. In the first phase, we build a collection of disjoint paths, connecting the cluster corresponding to the root of the tree $T$ to the clusters in $\mathcal{L}$, along the root-to-leaf paths in $T$. In the second phase, we build the path-of-sets system by exploiting the paths constructed in Step 1 to simulate the tree tour.

\subsection{Step 1}

Let $G^{\prime}$ be the graph obtained from the union of $G[S]$ for all $S \in \mathcal{S}$, and the sets $\mathcal{P}_{e}^{*}$ of paths, for all $e \in E(T)$. We root $T$ at a degree-1 vertex that does not belong to $L$ (since $T$ has at least $\ell^{*}+1$ leaves and $|L|=\ell^{*}$, such a vertex exists), and we let $S^{*}$ be the cluster corresponding to the root of $T$. The goal of the first step is summarized in the following theorem.

THEOREM 4.7. There is an efficient algorithm to compute, for each $S \in \mathcal{L}$, a collection $\mathcal{Q}_{S}$ of $\left\lfloor\tilde{w} / \ell^{*}\right\rfloor$ paths in graph $G^{\prime}$ that have the following properties:

- Each path $Q \in \mathcal{Q}_{S}$ starts at a vertex of $S^{*}$ and terminates at a vertex of $S$; its inner vertices are disjoint from $S$ and $S^{*}$.

-For each path $Q \in \mathcal{Q}_{S}$, for each cluster $S^{\prime} \in \mathcal{S}$, such that $v_{S^{\prime}}$ lies on the path connecting $v_{S^{*}}$ to $v_{S}$ in $T, Q \cap G\left[S^{\prime}\right]$ is a (nonempty) path. For all other clusters $S^{\prime} \in \mathcal{S}, Q \cap G\left[S^{\prime}\right]=$ $\emptyset$.

-The paths in $\mathcal{Q}=\bigcup_{S \in \mathcal{L}} \mathcal{Q}_{S}$ are vertex disjoint. 
Notice that from the structure of graph $G^{\prime}$, if $P$ is the path connecting $v_{S^{*}}$ to $v_{S}$ in the tree $T$, then every path in $\mathcal{Q}_{S}$ visits every cluster $S^{\prime}$ with $v_{S^{\prime}} \in P$ exactly once, in the order in which they appear on $P$, and it does not visit other clusters of $\mathcal{S}$.

Proof. Recall that for every vertex $v_{S} \in V(T)$, and for each edge $e$ incident to $v_{S}$, we have defined a subset $\delta_{S}(e) \subseteq S$ of vertices that serve as endpoints of the paths in $\mathcal{P}_{e}^{*}$. For each cluster $S^{\prime} \in \mathcal{S}$, let $n\left(S^{\prime}\right)$ be the number of the descendants of $v_{S^{\prime}}$ in the tree $T$ that belong to $L$. If $S^{\prime} \neq S^{*}$, then let $e$ be the edge of the tree $T$ connecting $v_{S^{\prime}}$ to its parent, and denote $\delta_{S^{\prime}}=\delta_{S^{\prime}}(e)$. We process the tree in top-to-bottom order while maintaining a set $\mathcal{Q}$ of disjoint paths. We ensure that the following invariant holds throughout the algorithm. Let $S, S^{\prime} \in \mathcal{S}$ be a pair of clusters such that $v_{S}$ is the parent of $v_{S^{\prime}}$ in $T$. Assume that so far the algorithm has processed $v_{S}$ but has not processed $v_{S^{\prime}}$ yet. Then there is a collection $\mathcal{Q}_{S^{\prime}} \subseteq \mathcal{Q}$ of $n\left(S^{\prime}\right) \cdot\left\lfloor\tilde{w} / \ell^{*}\right\rfloor$ paths connecting $S^{*}$ to $S^{\prime}$ in $\mathcal{Q}$. Each such path does not share vertices with $S^{\prime}$, except for its last vertex, which must belong to $\delta_{S^{\prime}}$. Moreover, for every path $Q \in \mathcal{Q}_{S^{\prime}}$, for every cluster $S^{\prime \prime} \in \mathcal{S}$, such that $v_{S^{\prime \prime}}$ lies on the path connecting $v_{S^{*}}$ to $v_{S^{\prime}}$ in $T, Q \cap G\left[S^{\prime \prime}\right]$ is a (nonempty) path, and for every other cluster $S^{\prime \prime}, Q \cap G\left[S^{\prime \prime}\right]=\emptyset$.

In the first iteration, we start with the root vertex $v_{S^{*}}$. Let $v_{S}$ be its unique child, and let $e=\left(v_{S^{*}}, v_{S}\right)$ be the corresponding edge of $T$. We let $\mathcal{Q}_{S}$ be an arbitrary subset of $n(S) \cdot\left\lfloor\tilde{w} / \ell^{*}\right\rfloor$ paths of $\mathcal{P}_{e}^{*}$, and we set $\mathcal{Q}=\mathcal{Q}_{S}$. (Notice that $|L| \cdot\left\lfloor\tilde{w} / \ell^{*}\right\rfloor \leq \tilde{w}=\left|\mathcal{P}_{e}^{*}\right|$, since $|L|=\ell^{*}$, so we can always find such a subset of paths.)

Consider now some nonleaf vertex $v_{S}$, and assume that its parent has already been processed. We assume that $v_{S}$ has two children. The case where $v_{S}$ has only one child is treated similarly. Let $\mathcal{Q}_{S} \subseteq \mathcal{Q}$ be the subset of paths currently connecting $S^{*}$ to $S$, and let $\Gamma^{\prime} \subseteq \delta_{S}$ be the endpoints of these paths that belong to $S$. Let $v_{S^{\prime}}, v_{S^{\prime \prime}}$ be the children of $v_{S}$ in $T$, and let $e_{1}=\left(v_{S}, v_{S^{\prime}}\right), e_{2}=\left(v_{S}, v_{S^{\prime \prime}}\right)$ be the corresponding edges of $T$. We need the following claim.

CLAIM 4.8. We can efficiently find a subset $\Gamma_{1} \subseteq \delta_{S}\left(e_{1}\right)$ of $n\left(S^{\prime}\right) \cdot\left\lfloor\tilde{w} / \ell^{*}\right\rfloor$ vertices and a subset $\Gamma_{2} \subseteq \delta_{S}\left(e_{2}\right)$ of $n\left(S^{\prime \prime}\right) \cdot\left\lfloor\tilde{w} / \ell^{*}\right\rfloor$ vertices, together with a set $\mathcal{R}$ of $\left|\Gamma^{\prime}\right|$ disjoint paths contained in $G[S]$, where each path connects a vertex of $\Gamma^{\prime}$ to a distinct vertex of $\Gamma_{1} \cup \Gamma_{2}$.

Proof. We build the following flow network, starting with $G[S]$. Set the capacity of every vertex in $S$ to 1 . Add a sink $t$, and connect every vertex in $\Gamma^{\prime}$ to $t$ with a directed edge. Add a new vertex $s_{1}$ of capacity $n\left(S^{\prime}\right) \cdot\left\lfloor\tilde{w} / \ell^{*}\right\rfloor$, and connect it with a directed edge to every vertex of $\delta_{S}\left(e_{1}\right)$. Similarly, add a new vertex $s_{2}$ of capacity $n\left(S^{\prime \prime}\right) \cdot\left\lfloor\tilde{w} / \ell^{*}\right\rfloor$, and connect it with a directed edge to every vertex of $\delta_{S}\left(e_{2}\right)$. Finally, add a source $s$, and connect it to $s_{1}$ and $s_{2}$ with directed edges. From the integrality of flow, it is enough to show that there is an $s$ - $t$ flow of value $\left|\Gamma^{\prime}\right|=n(S) \cdot\left\lfloor\tilde{w} / \ell^{*}\right\rfloor=\left(n\left(S^{\prime}\right)+n\left(S^{\prime \prime}\right)\right) \cdot\left\lfloor\tilde{w} / \ell^{*}\right\rfloor$ in this flow network. Since $\Gamma^{\prime}$ and $\delta_{S}\left(e_{1}\right)$ are linked, there is a set $\mathcal{P}_{1}$ of $\left|\Gamma^{\prime}\right|$ disjoint paths connecting the vertices of $\Gamma^{\prime}$ to the vertices of $\delta_{S}\left(e_{1}\right)$. We send $n\left(S^{\prime}\right) / n(S)$ flow units along each such path. Similarly, there is a set $\mathcal{P}_{2}$ of $\left|\Gamma^{\prime}\right|$ disjoint paths connecting vertices of $\Gamma^{\prime}$ to vertices of $\delta_{S}\left(e_{2}\right)$. We send $n\left(S^{\prime \prime}\right) / n(S)$ flow units along each such path. It is immediate to verify that this gives a feasible $s-t$ flow of value $\left|\Gamma^{\prime}\right|$ in this network.

Let $\mathcal{P}_{1} \subseteq \mathcal{P}^{*}\left(e_{1}\right)$ be the subset of paths whose endpoints belong to $\Gamma_{1}$, and define $\mathcal{P}_{2} \subseteq \mathcal{P}^{*}\left(e_{2}\right)$ similarly for $\Gamma_{2}$. Concatenating the paths in $\mathcal{Q}_{S}, \mathcal{R}$, and $\mathcal{P}_{1} \cup \mathcal{P}_{2}$, we obtain two collections of paths, set $\mathcal{Q}_{S^{\prime}}$ of $n\left(S^{\prime}\right) \cdot\left\lfloor\tilde{w} / \ell^{*}\right\rfloor$ paths, connecting $S^{*}$ to $S^{\prime}$, and set $\mathcal{Q}_{S^{\prime \prime}}$ of $n\left(S^{\prime \prime}\right) \cdot\left\lfloor\tilde{w} / \ell^{*}\right\rfloor$ paths, connecting $S^{*}$ to $S^{\prime \prime}$, that have the desired properties. We delete the paths of $\mathcal{Q}_{S}$ from $\mathcal{Q}$ and add the paths in $\mathcal{Q}_{S^{\prime}}$ and $\mathcal{Q}_{S^{\prime \prime}}$ instead.

Once all nonleaf vertices of the tree $T$ are processed, we obtain the desired collection of paths. 


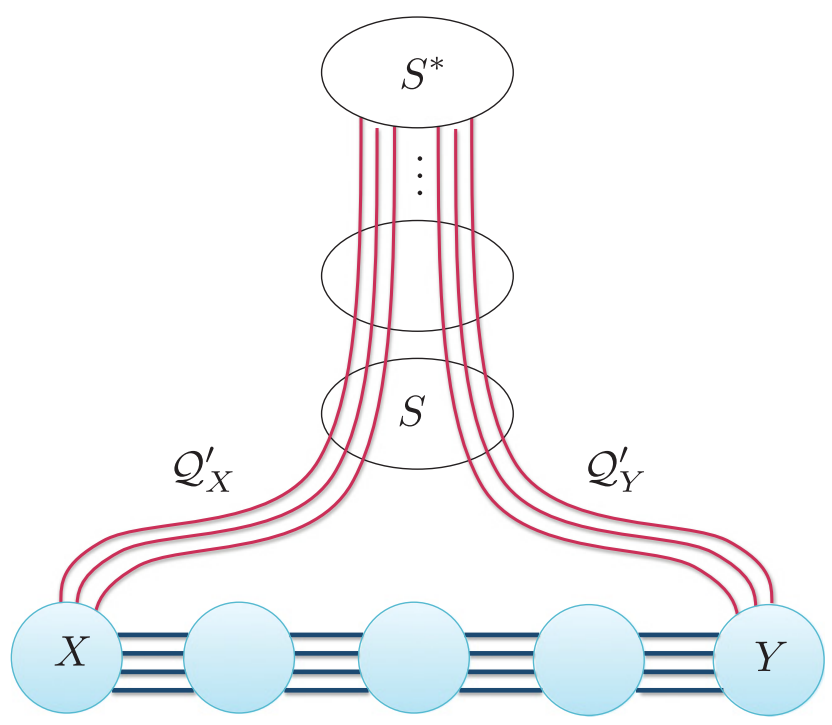

Fig. 3. Invariant for Step 2.

\subsection{Step 2}

In this step, we process the tree $T$ in the bottom-up order, gradually building the pathof-sets system. We will imitate the DFS tour of the tree and exploit the sets $\left\{\mathcal{Q}_{S} \mid S \in \mathcal{L}\right\}$ of paths constructed in Step 1 to perform this step.

For every vertex $v_{S}$ of the tree $T$, let $T_{v_{S}}$ be the subtree of $T$ rooted at $v_{S}$. Define a subgraph $G_{S}$ of $G^{\prime}$ to be the union of all clusters $G^{\prime}\left[S^{\prime}\right]$ with $v_{S^{\prime}} \in V\left(T_{v_{S}}\right)$ and all sets $\mathcal{P}_{e}^{*}$ of paths with $e \in E\left(T_{v_{S}}\right)$. We also define $L_{S} \subseteq L$ to be the set of all descendants of $v_{S}$ that belong to $L$, and $\mathcal{L}_{S}=\left\{S^{\prime} \mid v_{S^{\prime}} \in L_{S}\right\}$ the collection of the corresponding clusters.

We process the tree $T$ in a bottom-to-top order, maintaining the following invariant. Let $v_{S}$ be a vertex of $T$, and let $\ell_{S}$ be the length of the longest simple path connecting $v_{S}$ to its descendant in $T$. Once vertex $v_{S}$ is processed, we have computed a path-ofsets system $\left(\mathcal{L}_{S}, \mathcal{P}^{S}\right)$ of width $w^{*}$ and length $\left|\mathcal{L}_{S}\right|$ that is completely contained in $G_{S}$. (In other words, the path-of-sets system is defined over the collection $\mathcal{L}_{S}$ of vertex subsets-all subsets $S^{\prime} \in \mathcal{L}$ where $v_{S^{\prime}}$ is a descendant of $v_{S}$ in $T$.) Let $X, Y \in \mathcal{L}_{S}$ be the first and the last set on the path-of-sets system. Then we also compute subsets $\mathcal{Q}_{X}^{\prime} \subseteq \mathcal{Q}_{X}, \mathcal{Q}_{Y}^{\prime} \subseteq \mathcal{Q}_{Y}$ of paths of cardinality at least $\left\lfloor\frac{\tilde{w}}{2 \ell^{*}}\right\rfloor-8 \ell_{S} \cdot w^{*}$ such that the paths in $\mathcal{Q}_{X}^{\prime} \cup \mathcal{Q}_{Y}^{\prime}$ are completely disjoint from the paths in $\mathcal{P}^{S}$ (Figure 3). Note that $\mathcal{Q}_{X}, \mathcal{Q}_{Y}$ are the sets of paths computed in Step 1, so the paths in $\mathcal{Q}_{X} \cup \mathcal{Q}_{Y}$ are also disjoint from $\bigcup_{S^{\prime} \in \mathcal{L}} S^{\prime}$, except one endpoint of each such path must belong to $X$ or $Y$. We note that since the tree height is less than $\ell^{*},\left\lfloor\frac{\tilde{w}}{2 \ell^{*}}\right\rfloor-8 \ell_{S} \cdot w^{*}>\left\lfloor\frac{\tilde{w}}{2 \ell^{*}}\right\rfloor-8 \ell^{*} \cdot w^{*} \geq 0$, where the latter inequality is based on the assumption that $\tilde{w}>16 w^{*}\left(\ell^{*}\right)^{2}+1$.

Clearly, once all vertices of the tree $T$ are processed, we obtain the desired path-ofsets system $\left(\mathcal{L}, \mathcal{P}, A_{1}, B_{\ell^{*}}\right)$ of length $\ell^{*}$ and width $w^{*}$. We now describe the algorithm for processing each vertex.

If $v_{S}$ is a leaf of $T$, then we do nothing. If $v_{S} \in L$, then the path-of-sets system consists of only $\mathcal{S}=\{S\}$, with $X=Y=S$. We let $\mathcal{Q}_{X}^{\prime}, \mathcal{Q}_{Y}^{\prime}$ be an arbitrary pair of disjoint subsets of $\mathcal{Q}_{S}$ containing $\left\lfloor\frac{\tilde{w}}{2 \ell^{*}}\right\rfloor$ paths each. If $v_{S}$ is a degree-2 vertex of $T$, then we also do nothing. The path-of-sets system is inherited from its child, and the corresponding sets $\mathcal{Q}_{X}^{\prime}, \mathcal{Q}_{Y}^{\prime}$ remain unchanged. Assume now that $v_{S}$ is a degree-3 vertex, and let $v_{S^{\prime}}, v_{S^{\prime \prime}}$ be 
its two children. Consider the path-of-sets systems that we computed for its children: $\left(\mathcal{L}_{S^{\prime}}, \mathcal{P}^{S^{\prime}}\right)$ for $S^{\prime}$ and $\left(\mathcal{L}_{S^{\prime \prime}}, \mathcal{P}^{S^{\prime \prime}}\right)$ for $S^{\prime \prime}$. Let $X_{1}, Y_{1}$ be the first and the last cluster of the first system and $X_{2}, Y_{2}$ the first and the last cluster of the second system (Figure 4(a)). The idea is to connect the two path-of-sets systems into a single system by joining one of $\left\{X_{1}, Y_{1}\right\}$ to one of $\left\{X_{2}, Y_{2}\right\}$ by $w^{*}$ disjoint paths. These paths are constructed by concatenating subpaths of some paths from $\mathcal{Q}_{X_{1}}^{\prime} \cup \mathcal{Q}_{Y_{1}}^{\prime} \cup \mathcal{Q}_{X_{2}}^{\prime} \cup \mathcal{Q}_{Y_{2}}^{\prime}$ and additional paths contained in $G[S]$.

Consider the paths in $\mathcal{Q}_{X_{1}}^{\prime}$ and direct these paths from $X_{1}$ toward $S^{*}$. For each such path $Q$, let $v_{Q}$ be the first vertex of $Q$ that belongs to $S$. Let $\Gamma_{1}=\left\{v_{Q} \mid Q \in \mathcal{Q}_{X_{1}}^{\prime}\right\}$. We similarly define $\Gamma_{2}, \Gamma_{1}^{\prime}$, and $\Gamma_{2}^{\prime}$ for $\mathcal{Q}_{Y_{1}}^{\prime}, \mathcal{Q}_{X_{2}}^{\prime}$, and $\mathcal{Q}_{Y_{2}}^{\prime}$, respectively. Denote $\Gamma=\Gamma_{1} \cup \Gamma_{2}$, and $\Gamma^{\prime}=\Gamma_{1}^{\prime} \cup \Gamma_{2}^{\prime}$. For simplicity, we denote the portions of the paths in $\mathcal{Q}_{X_{1}}^{\prime} \cup \mathcal{Q}_{Y_{1}}^{\prime}$ that are contained in $G[S]$ by $\mathcal{P}$ and the portions of paths in $\mathcal{Q}_{X_{2}}^{\prime} \cup \mathcal{Q}_{Y_{2}}^{\prime}$ that are contained in $G[S]$ by $\mathcal{P}^{\prime}$ (see Figure $4(\mathrm{~b})$ ). In other words,

$$
\mathcal{P}=\left\{P \cap G[S] \mid P \in \mathcal{Q}_{X_{1}}^{\prime} \cup \mathcal{Q}_{Y_{1}}^{\prime}\right\} ; \quad \mathcal{P}^{\prime}=\left\{P \cap G[S] \mid P \in \mathcal{Q}_{X_{2}}^{\prime} \cup \mathcal{Q}_{Y_{2}}^{\prime}\right\} .
$$

Our goal is to find a set $\mathcal{R}$ of $4 w^{*}$ disjoint paths in $G[S]$ connecting $\Gamma$ to $\Gamma^{\prime}$ such that the paths in $\mathcal{R}$ intersect at most $8 w^{*}$ paths in $\mathcal{P}$ and at most $8 w^{*}$ paths in $\mathcal{P}^{\prime}$. Notice that, in general, since sets $\Gamma, \Gamma^{\prime}$ are linked in $G[S]$, we can find a set $\mathcal{R}$ of $4 w^{*}$ disjoint paths in $G[S]$ connecting $\Gamma$ to $\Gamma^{\prime}$, but these paths may intersect many paths in $\mathcal{P} \cup \mathcal{P}^{\prime}$. We start from an arbitrary set $\mathcal{R}$ of $4 w^{*}$ disjoint paths connecting $\Gamma$ to $\Gamma^{\prime}$ in $G[S]$. We next reroute these paths using Lemma 2.19.

We apply Lemma 2.19 twice. First, we unify all vertices of $\Gamma$ into a single vertex $s$, and direct the paths in $\mathcal{P}$ and the paths in $\mathcal{R}$ toward it. We then apply Lemma 2.19 to the two sets of paths, with $\mathcal{P}$ as $\mathcal{X}_{1}$ and $\mathcal{R}$ as $\mathcal{X}_{2}$. Let $\tilde{\mathcal{P}} \subseteq \mathcal{P}, \mathcal{R}^{\prime}$ be the two resulting sets of paths. We discard from $\tilde{\mathcal{P}}$ paths that share endpoints with paths in $\mathcal{R}^{\prime}$ (at most $\left|\mathcal{R}^{\prime}\right|$ paths). Then $|\tilde{\mathcal{P}}| \geq|\mathcal{P}|-2|\mathcal{R}|=|\mathcal{P}|-8 w^{*}$, and $\mathcal{R}^{\prime}$ contains $4 w^{*}$ disjoint paths connecting vertices in $\Gamma$ to vertices in $\Gamma^{\prime}$. Moreover, the paths in $\tilde{\mathcal{P}} \cup \mathcal{R}^{\prime}$ are completely disjoint.

Next, we unify all vertices in $\Gamma^{\prime}$ into a single vertex $s$, and direct all paths in $\mathcal{P}^{\prime}$ and $\mathcal{R}^{\prime}$ toward $s$. We then apply Lemma 2.19 to the two resulting sets of paths, with $\mathcal{P}^{\prime}$ serving as $\mathcal{X}_{1}$ and $\mathcal{R}^{\prime}$ serving as $\mathcal{X}_{2}$. Let $\tilde{\mathcal{P}}^{\prime} \subseteq \mathcal{P}^{\prime}$ and $\mathcal{R}^{\prime \prime}$ be the two resulting sets of paths. We again discard from $\tilde{\mathcal{P}}^{\prime}$ all paths that share an endpoint with a path in $\mathcal{R}^{\prime \prime}$-at most $\left|\mathcal{R}^{\prime \prime}\right|$ paths. Then $\left|\tilde{\mathcal{P}}^{\prime}\right| \geq\left|\mathcal{P}^{\prime}\right|-2\left|\mathcal{R}^{\prime \prime}\right| \geq\left|\mathcal{P}^{\prime}\right|-8 w^{*}$, and the paths in $\tilde{\mathcal{P}}^{\prime} \cup \mathcal{R}^{\prime \prime}$ are completely disjoint from each other. Notice also that the paths in $\mathcal{R}^{\prime \prime}$ remain disjoint from the paths in $\tilde{\mathcal{P}}$, as the paths in $\mathcal{R}^{\prime \prime}$ only use vertices that appear on the paths in $\mathcal{R}^{\prime} \cup \mathcal{P}^{\prime}$, which are disjoint from $\tilde{\mathcal{P}}$.

Consider now the final set $\mathcal{R}^{\prime \prime}$ of paths. The paths in $\mathcal{R}^{\prime \prime}$ connect the vertices of $\Gamma_{1} \cup \Gamma_{2}$ to the vertices of $\Gamma_{1}^{\prime} \cup \Gamma_{2}^{\prime}$. There must be two indices, $i, j \in\{1,2\}$, such that at least a quarter of the paths in $\mathcal{R}^{\prime \prime}$ connect vertices of $\Gamma_{i}$ to vertices of $\Gamma_{j}^{\prime}$. We assume without loss of generality that $i=2, j=1$, so at least $w^{*}$ of the paths in $\mathcal{R}^{\prime \prime}$ connect vertices of $\Gamma_{2}$ to vertices of $\Gamma_{1}^{\prime}$. Let $\mathcal{R}^{*} \subseteq \mathcal{R}^{\prime \prime}$ be the set of these paths. We obtain a collection $\mathcal{P}^{*}$ of $w^{*}$ paths connecting $Y_{1}$ to $X_{2}$ by concatenating the prefixes of the paths in $\mathcal{Q}_{Y_{1}}^{\prime}$, the paths in $\mathcal{R}^{\prime \prime}$, and the prefixes of the paths in $\mathcal{Q}_{X_{2}}^{\prime}$ (see Figure $4(\mathrm{c})$ ). Notice that the paths in $\mathcal{P}^{*}$ are completely disjoint from the two path-of-sets systems, except for their endpoints that belong to $Y_{1}$ and $X_{2}$. This gives us a new path-of-sets system, whose collection of vertex sets is $\mathcal{S}=\mathcal{L}_{S}$. The first and the last sets in this system are $X_{1}$ and $Y_{2}$, respectively. To define the new set $\mathcal{Q}_{X_{1}}^{\prime}$, we discard from $\mathcal{Q}_{X_{1}}^{\prime}$ all paths that share vertices with paths in $\mathcal{R}^{\prime \prime}$ (as observed before, there are at most $8 w^{*}$ such paths). Since at the beginning of the current iteration $\left|\mathcal{Q}_{X_{1}}^{\prime}\right| \geq\left\lfloor\frac{\tilde{w}}{2 \ell^{*}}\right\rfloor-8 w^{*} \ell_{S^{\prime}} \geq\left\lfloor\frac{\tilde{w}}{2 \ell^{*}}\right\rfloor-8 w^{*}\left(\ell_{S}-1\right)$, at the end of the current iteration $\left|\mathcal{Q}_{X_{1}}^{\prime}\right| \geq\left\lfloor\frac{\tilde{w}}{2 \ell^{*}}\right\rfloor-8 w^{*} \ell_{S}$, as required. The new set $\mathcal{Q}_{Y_{2}}^{\prime}$ is defined similarly. From the construction, the paths in $\mathcal{Q}_{X_{1}}^{\prime} \cup \mathcal{Q}_{Y_{2}}^{\prime}$ are completely 


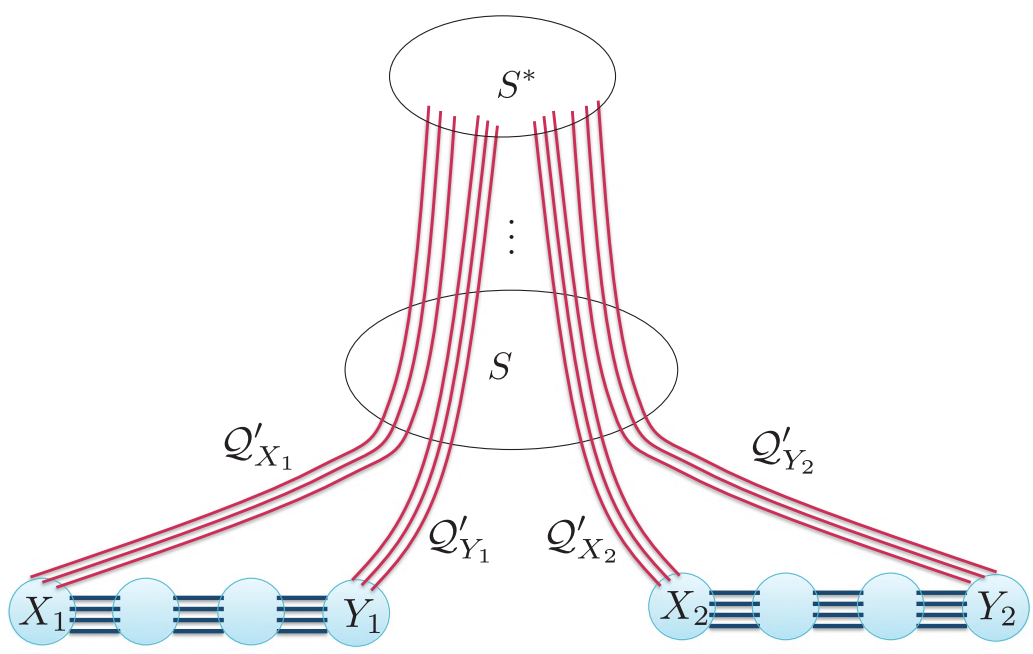

(a) The beginning

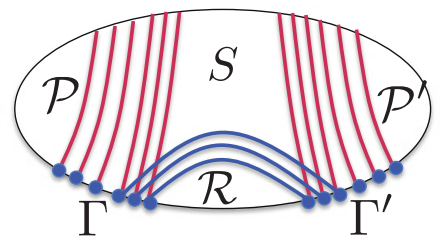

(b) Finding the set $\mathcal{R}$ of paths

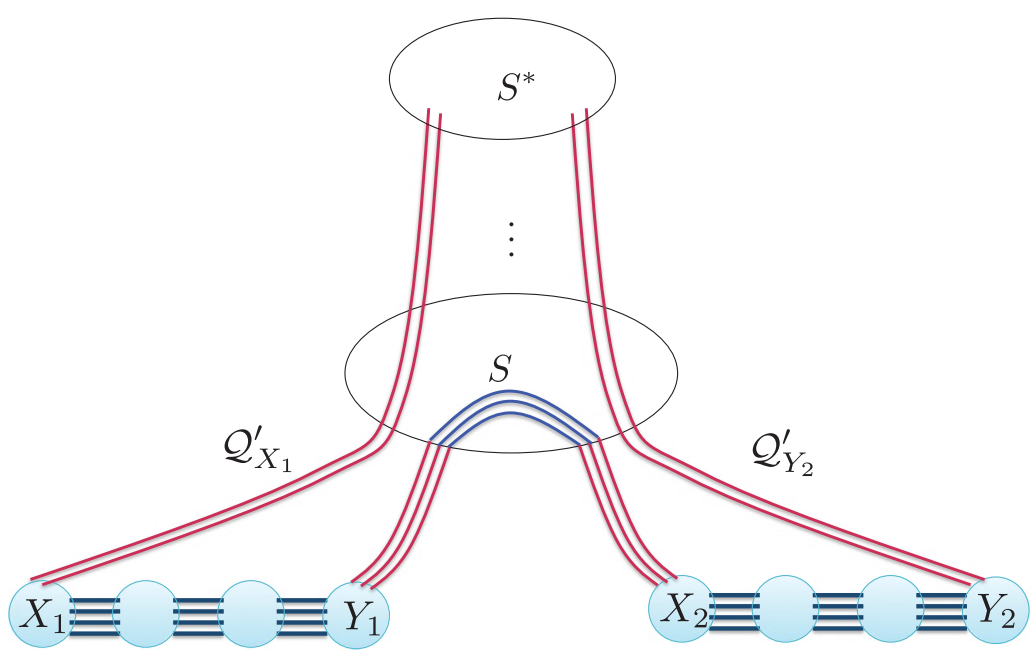

(c) The end

Fig. 4. Processing a degree-3 vertex $v_{S}$. 
disjoint from the paths in $\mathcal{R}^{*}$, and hence they are completely disjoint from all paths participating in the new path-of-sets system.

Notice that each vertex $v_{i} \in L$ is only incident on one edge $e \in E(T)$, and from the definition of strong tree-of-sets system, $\delta_{S_{i}}(e)$ is node-well-linked in $G\left[S_{i}\right]$. These are the only vertices of $S_{i}$ that may participate in the paths $\mathcal{P}_{j}$ of the path-of-sets system, so we obtain a strong path-of-sets system.

To complete the proof of Theorem 1.1, it now suffices to prove Theorem 4.3.

\section{PROOF OF THEOREM 4.3}

This section mostly follows the algorithm of Chuzhoy and Li [2016]. The main difference is a change in the parameters so that the number of clusters in the tree-of-sets system is polynomial in $k$ and not polylogarithmic, and extending the arguments of Chuzhoy and $\mathrm{Li}$ [2016] to handle vertex connectivity instead of edge connectivity. We also improve and simplify some of the arguments of Chuzhoy and Li [2016]. Some of the proofs and definitions are identical to or closely follow those in Chuzhoy and Li [2016] and are provided here for the sake of completeness. For simplicity, if $\left(\mathcal{S}, T, \bigcup_{e \in E(T)} \mathcal{P}_{e}\right)$ is a tree-of-sets system in $G$, with parameters $w, \ell, \alpha_{\mathrm{BW}}$ as in the theorem statement, and for each $S_{i} \in \mathcal{S}, S_{i} \cap \mathcal{T}=\emptyset$, then we say that it is a good tree-of-sets system.

\subsection{High-Level Overview}

We now provide a high-level overview and intuition for the proof of Theorem 4.3. We also describe a nonconstructive proof of the theorem, which is somewhat simpler than the constructive proof that appears in the following. This high-level description oversimplifies some parts of the algorithm for the sake of clarity. This section is not necessary for understanding the algorithm and is only provided for the sake of intuition. A formal self-contained proof appears in the following sections.

Recall that the starting point is a graph $G=(V, E)$ and a set $\mathcal{T} \subseteq V$ of $k$ terminals such that $\mathcal{T}$ is node-well-linked in $G$. Set $\mathcal{T}$ certifies that $G$ has treewidth $\Omega(k)$. There can be portions of the graph that are not well connected to $\mathcal{T}$ and hence are irrelevant to its well-linkedness property. We can assume without loss of generality that $G$ is edge minimal subject to satisfying the condition that $\mathcal{T}$ is node-well-linked. However, there is no easy structural or algorithmic way to characterize this minimality condition. For this reason, in various parts of the proof, we will delete or suppress irrelevant portions of the graph. Recall that the goal is to prove that given $G$ and $\mathcal{T}$, there is a tree-ofsets system with appropriate parameters. Loosely speaking, a tree-of-sets system with parameters $\ell, w, \alpha_{\mathrm{BW}}$ consists of $\ell$ vertex-disjoint subgraphs with vertex sets $S_{1}, \ldots, S_{\ell}$ stitched together with collections of paths in a tree-like fashion. From the definition, we note that each $S_{i}$ has the property that $G\left[S_{i}\right]$ contains a well-linked vertex set of size $\Omega\left(\alpha_{\mathrm{BW}} \cdot w / \Delta\right)$. Thus, we need as a building block, a procedure that allows us to take a graph $G$ with a well-linked set of size $k$ and decomposes $G$ into $\ell$ disjoint subgraphs each of which has a well-linked set of size $\Omega\left(\alpha_{\mathrm{BW}} \cdot w / \Delta\right)$. The fact that this can be done was first shown in Chuzhoy [2016] and stated explicitly with additional refinements in Chekuri and Chuzhoy [2013]. We make the discussion more precise in the following.

The proof uses two main parameters: $\ell_{0}=\ell^{2}$, and $w_{0}=w \cdot \operatorname{poly}(\ell \cdot \Delta \cdot \log k)$. We say that a subset $S$ of vertices of $G$ is a good router if and only if the following three conditions hold: (1) $S \cap \mathcal{T}=\emptyset$, (2) $S$ has the $\alpha_{\mathrm{BW}}$-bandwidth property, and (3) $S$ can send a large amount of flow (say at least $w_{0} / 2$ flow units) to $\mathcal{T}$ with no edge congestion in $G$. A collection of $\ell_{0}$ disjoint good routers is called a good family of routers. Roughly, the proof consists of two parts. The first part shows how to find a good family of routers, and the second part shows that, given a good family routers, we can build a good tree-of-sets system. We start by describing the second part, which is somewhat simpler. 
From a good family of routers to a good tree-of-sets system. Suppose that we are given a good family $\mathcal{R}=\left\{S_{1}, \ldots, S_{\ell_{0}}\right\}$ of routers. We now give a high-level description of an algorithm to construct a good tree-of-sets system from $\mathcal{R}$ (a formal proof appears in Section 5.4). The algorithm consists of two phases. We start with the first phase.

Since every set $S_{i} \in \mathcal{R}$ can send $w_{0} / 2$ flow units to the terminals with no edge congestion, and the terminals are 1-well-linked in $G$, it is easy to see that every pair $S_{i}, S_{j} \in \mathcal{R}$ of sets can send $w_{0} / 2$ flow units to each other with edge congestion at most 3 , and so there are at least $\frac{w_{0}}{6 \Delta}$ node-disjoint paths connecting $S_{i}$ to $S_{j}$. We build an auxiliary graph $H$ from $G$ by contracting each cluster $S_{i} \in \mathcal{R}$ into a supernode $v_{i}$. We view the supernodes $v_{1}, \ldots, v_{\ell_{0}}$ as the terminals of $H$ and denote $\tilde{\mathcal{T}}=\left\{v_{1}, \ldots, v_{\ell_{0}}\right\}$. We then use standard splitting procedures in graph $H$ repeatedly to obtain a new graph $H^{\prime}$, whose vertex set is $\tilde{\mathcal{T}}$, every pair of vertices remains $\frac{w_{0}}{\operatorname{poly}(\Delta)}$-edge connected, and every edge $e=\left(v_{i}, v_{j}\right) \in E\left(H^{\prime}\right)$ corresponds to a path $P_{e}$ in $G$, connecting a vertex of $S_{i}$ to a vertex of $S_{j}$. Moreover, the paths $\left\{P_{e} \mid e \in E\left(H^{\prime}\right)\right\}$ are node disjoint, and they do not contain the vertices of $\bigcup_{S \in \mathcal{R}} S$ as inner vertices. More specifically, graph $H$ is obtained from $H^{\prime}$ by first performing a sequence of edge contraction and edge deletion steps that preserve element connectivity of the terminals and then performing standard edgesplitting steps that preserves edge connectivity. Let $Z$ be a graph whose vertex set is $\tilde{\mathcal{T}}$, and there is an edge $\left(v_{i}, v_{j}\right)$ in $Z$ if and only if there are many (say $\left.\frac{w_{0}}{\ell_{0}^{2} \text { poly }(\Delta)}\right)$ parallel edges $\left(v_{i}, v_{j}\right)$ in $H^{\prime}$. We show that $Z$ is a connected graph, and so we can find a spanning tree $T$ of $Z$. Since $\ell_{0}=\ell^{2}$, either $T$ contains a path of length $\ell$ or contains at least $\ell$ leaves. Consider the first case, where $T$ contains a path $P$ of length $\ell$. We can use the path $P$ to define a tree-of-sets system (in fact, it will give a path-of-sets system directly, after we apply Theorem 2.14 to boost the well-linkedness of the boundaries of the clusters that participate in $P$ and Theorem 2.9 to ensure the linkedness of the corresponding vertex subsets inside each cluster). From now on, we focus on the second case, where $T$ contains $\ell$ leaves. Assume without loss of generality that the good routers associated with the leaves of $T$ are $\mathcal{R}^{\prime}=\left\{S_{1}, \ldots, S_{\ell}\right\}$. We show that we can find, for each $1 \leq i \leq \ell$, a subset $E_{i} \subseteq \operatorname{out}_{G}\left(S_{i}\right)$ of $w_{3}=w \operatorname{poly}(\ell \cdot \Delta)$ edges such that for each pair $1 \leq i<j \leq \ell$, there are $w_{3}$ node-disjoint paths connecting $S_{i}$ to $S_{j}$ in $G$, where each path starts with an edge of $E_{i}$ and ends with an edge of $E_{j}$. To compute the sets $E_{i}$ of edges, we show that we can simultaneously connect each set $S_{i}$ to the set $S^{*} \in \mathcal{R}$ corresponding to the root of tree $T$ with many paths. For each $i$, let $\mathcal{P}_{i}$ be the collection of paths connecting $S_{i}$ to $S^{*}$. We will ensure that all paths in $\bigcup_{i} \mathcal{P}_{i}$ are node disjoint. The existence of the sets $\mathcal{P}_{i}$ of paths follows from the fact that all sets $S_{i}$ can simultaneously send large amounts of flow to $S^{*}$ (along the leaf-to-root paths in the tree $T$ ) with relatively small congestion. After boosting the well-linkedness of the endpoints of these paths in $S^{*}$ using Theorem 2.14 for each $\mathcal{P}_{i}$ separately, and ensuring that for every pair $\mathcal{P}_{i}, \mathcal{P}_{j}$ of such path sets their endpoints are linked inside $S^{*}$ using Theorem 2.9, we obtain somewhat smaller subsets $\mathcal{P}_{i}^{\prime} \subseteq \mathcal{P}_{i}$ of paths for each $i$. The desired set $E_{i}$ of edges is obtained by taking the first edge on every path in $\mathcal{P}_{i}^{\prime}$. We now proceed to the second phase.

The execution of the second phase is quite similar to the execution of the first phase, except the initial graph $H$ is built slightly differently. We will ignore the clusters in $\mathcal{R} \backslash \mathcal{R}^{\prime}$. For each cluster $S_{i} \in \mathcal{R}^{\prime}$, we delete all edges in $\operatorname{out}_{G}\left(S_{i}\right) \backslash E_{i}$ from $G$ and then contract the vertices of $S_{i}$ into a supernode $v_{i}$. As before, we consider the set $\tilde{\mathcal{T}}=\left\{v_{1}, \ldots, v_{\ell}\right\}$ of supernodes to be the terminals of the resulting graph $\tilde{H}$. Observe that now the degree of every terminal $v_{i}$ is exactly $w_{3}$, and the edge connectivity between every pair of terminals is also exactly $w_{3}$. It is this additional property that allows us to build the tree-of-sets system in this phase. As before, we perform standard splitting operations to reduce graph $\tilde{H}$ to a new graph $\tilde{H}^{\prime}$, whose vertex set is $\tilde{\mathcal{T}}$. As before, every 
edge $e=\left(v_{i}, v_{j}\right)$ in $\tilde{H}^{\prime}$ corresponds to a path $P_{e}$ connecting a vertex of $S_{i}$ to a vertex of $S_{j}$ in $G$; all paths in $\left\{P_{e} \mid e \in E\left(\tilde{H}^{\prime}\right)\right\}$ are node disjoint, and they do not contain the vertices of $\bigcup_{S \in \mathcal{R}^{\prime}} S$ as inner vertices. However, we now have the additional property that the degree of every vertex $v_{i}$ in $\tilde{H}^{\prime}$ is $w_{3}$, and the edge connectivity of every pair of vertices is also $w_{3}$. We build a graph $\tilde{Z}$ on the set $\tilde{\mathcal{T}}$ of vertices as follows: for every pair $\left(v_{i}, v_{j}\right)$ of vertices, if their number of edges $\left(v_{i}, v_{j}\right)$ in $\tilde{H}^{\prime}$ is $n_{i, j}>w_{3} / \ell^{3}$, then we add $n_{i, j}$ parallel edges $\left(v_{i}, v_{j}\right)$ to $\tilde{Z}$. Otherwise, if $n_{i, j}<w_{3} / \ell^{3}$, then we do not add an edge connecting $v_{i}$ to $v_{j}$. We then show that the degree of every vertex in $\tilde{Z}$ remains very close to $w_{3}$, and the same holds for edge connectivity of every pair of vertices in $\tilde{Z}$. Note that every pair $v_{i}, v_{j}$ of vertices of $\tilde{Z}$ is either connected by many parallel edges or there is no edge $\left(v_{i}, v_{j}\right)$ in $\tilde{Z}$. In the final step, we show that we can construct a spanning tree of $\tilde{Z}$ with maximum vertex degree bounded by 3 . This spanning tree immediately defines a good tree-of-sets system. The construction of the spanning tree is performed using a result of Singh and Lau [2015], who showed an approximation algorithm for constructing a minimum-degree spanning tree of a graph. Their algorithm is based on an LP-relaxation of the problem. They show that given a feasible solution to the LP-relaxation, one can construct a spanning tree with maximum degree bounded by the maximum fractional degree plus 1 . Therefore, it is enough to show that there is a solution to the LP-relaxation on graph $\tilde{Z}$, where the fractional degree of every vertex is bounded by 2 . The fact that the degree of every vertex and the edge connectivity of every pair of vertices are very close to the same value allows us to construct such a solution.

An alternative way of seeing that graph $\tilde{Z}$ has a spanning tree of degree at most 3 is to observe that graph $\tilde{Z}$ is 1 -tough (i.e., if we remove $q$ vertices from $\tilde{Z}$, there are at most $q$ connected components in the resulting graph for every $q$ ). It is known that a 1-tough graph has a spanning tree of degree at most 3 [Win 1989].

Finding a good family of routers. One of the main tools that we use in this part is a good clustering of the graph $G$ and a legal contracted graph associated with it. We say that a subset $C \subseteq V(G)$ of vertices is a small cluster if and only if $|\operatorname{out}(C)| \leq w_{0}$, and we say that it is a large cluster otherwise. A partition $\mathcal{C}$ of $V(G)$ is called a good clustering if and only if each terminal $t \in \mathcal{T}$ belongs to a separate cluster $C_{t} \in \mathcal{C}$, where $C_{t}=\{t\}$, all clusters in $\mathcal{C}$ are small, and each cluster has the $\alpha_{\mathrm{BW}}$-bandwidth property. Given a good clustering $\mathcal{C}$, the corresponding legal contracted graph is obtained from $G$ by contracting every cluster $C \in \mathcal{C}$ into a supernode $v_{C}$ (notice that terminals are not contracted, as each terminal is in a separate cluster). The legal contracted graph can be seen as a model of $G$, where we "hide" some irrelevant parts of the graph inside the contracted clusters. The main idea of the algorithm is to exploit the legal contracted graph to find a good family of routers, and, if we fail to do so, to construct a smaller legal contracted graph. We start with a nonconstructive proof of the existence of a good family of routers in $G$.

Nonconstructive proof. We assume that $G$ is minimal inclusion-wise, for which the set $\mathcal{T}$ of terminals is 1 -well-linked. In other words, for an edge $e \in E(G)$, if we delete $e$ from $G$, then $\mathcal{T}$ is not 1 -well-linked in the resulting graph. Let $\mathcal{C}^{*}$ be a good clustering of $V(G)$ minimizing the total number of edges in the corresponding legal contracted graph (notice that a partition where every vertex belongs to a separate cluster is a good clustering, so such a clustering exists). Consider the resulting legal contracted graph $G^{\prime}$. The degree of every vertex in $G^{\prime}$ is at most $w_{0}$, and from the well-linkedness of the terminals in $G$, it is not hard to show that $G^{\prime} \backslash \mathcal{T}$ must contain at least $\Omega(k)$ edges. Then there is a partition $\left\{X_{1}, \ldots, X_{\ell_{0}}\right\}$ of $V\left(G^{\prime}\right) \backslash \mathcal{T}$, where for each $1 \leq i \leq \ell_{0}$, 
$\mid$ out $_{G^{\prime}}\left(X_{i}\right) \mid<O\left(\ell_{0}\left|E_{G^{\prime}}\left(X_{i}\right)\right|\right)$ (a random partition of $V\left(G^{\prime}\right) \backslash \mathcal{T}$ into $\ell_{0}$ subsets will have this property with constant probability because if we denote $m=\left|E\left(G^{\prime}\right) \backslash \mathcal{T}\right|$, then we expect roughly $\frac{m+k}{\ell_{0}}$ edges in set out $G_{G^{\prime}}\left(X_{i}\right)$ and roughly $m / \ell_{0}^{2}$ edges with both endpoints inside $X_{i}$ ).

For each set $X_{i}$, let $X_{i}^{\prime} \subseteq V(G) \backslash \mathcal{T}$ be the corresponding subset of vertices of $G$ obtained by uncontracting each supernode $v_{C}$ (i.e., $X_{i}^{\prime}=\bigcup_{v_{C} \in X_{i}} C$ ). If $\Gamma_{i}$ is the interface of $X_{i}^{\prime}$ in $G$, then we still have that $\left|\Gamma_{i}\right| \leq O\left(\ell_{0}\left|E_{G^{\prime}}\left(X_{i}\right)\right|\right)$. As our next step, we would like to find a partition $\mathcal{W}_{i}$ of the vertices of $X_{i}^{\prime}$ into clusters such that each cluster $W \in \mathcal{W}_{i}$ has the $\alpha_{\mathrm{BW}}$-bandwidth property and the total number of edges connecting different clusters is at most $O\left(\left|\Gamma_{i}\right| / \ell_{0}\right)<\left|E_{G^{\prime}}\left(X_{i}\right)\right|$. We call this procedure bandwidth decomposition. Assume first that we are able to find such a decomposition. We claim that $\mathcal{W}_{i}$ must contain at least one good router $S_{i}$. If this is the case, then we have found the desired family $\left\{S_{1}, \ldots, S_{\ell_{0}}\right\}$ of good routers. To show that $\mathcal{W}_{i}$ contains a good router, assume first that at least one cluster $S_{i} \in \mathcal{W}_{i}$ is large. The decomposition $\mathcal{W}_{i}$ already guarantees that $S_{i}$ has the $\alpha_{\mathrm{BW}}$-bandwidth property. If $S_{i}$ is not a good router, then it must be impossible to send large amounts of flow from $S_{i}$ to $\mathcal{T}$ in $G$. In this case, using known techniques (see the appendix to Chekuri et al. [2013b]), we can show that we can delete an edge from $G\left[S_{i}\right]$ while preserving the 1 -well-linkedness of the terminals, ${ }^{6}$ contradicting the minimality of $G$. Therefore, if $\mathcal{W}_{i}$ contains at least one large cluster, then it contains a good router. Assume now that all clusters in $\mathcal{W}_{i}$ are small. Then we show a new good clustering $\mathcal{C}^{\prime}$ of $V(G)$, whose corresponding contracted graph contains fewer edges than $G^{\prime}$, leading to a contradiction. The new clustering contains all clusters $C \in \mathcal{C}^{*}$ with $C \cap X_{i}^{\prime}=\varnothing$, and all clusters in $\mathcal{W}_{i}$. In other words, we replace the clusters contained in $X_{i}^{\prime}$ with the clusters of $\mathcal{W}_{i}$. The number of edges goes down in the legal contracted graph because the total number of edges connecting different clusters of $\mathcal{W}_{i}$ is less than $\left|E_{G^{\prime}}\left(X_{i}\right)\right|$.

The final part of the proof that we need to describe is the bandwidth-decomposition procedure. Given a cluster $X_{i}^{\prime}$, we would like to find a partition $\mathcal{W}_{i}$ of $X_{i}^{\prime}$ into clusters that have the $\alpha_{\mathrm{BW}}$-bandwidth property such that the number of edges connecting different clusters is bounded by $O\left(\left|\Gamma_{i}\right| / \ell_{0}\right)$. There are by now standard algorithms for finding such a decomposition, where we repeatedly select a cluster in $\mathcal{W}_{i}$ that does not have the desired bandwidth property and partition it along a sparse cut [Räcke 2002; Chekuri et al. 2005]. Unfortunately, since our bandwidth parameter $\alpha_{\mathrm{BW}}$ is independent of $n$, such an approach can only work when $\left|\Gamma_{i}\right|$ is bounded by poly $(k)$, which is not necessarily true in our case. To overcome this difficulty, as was done in Chuzhoy [2016], we slightly weaken the bandwidth condition and define a $\left(k, \alpha_{\mathrm{BW}}\right)$-bandwidth property as follows. We say that cluster $C$ with interface $\Gamma$ has the $\left(k, \alpha_{\mathrm{BW}}\right)$-bandwidth property if and only if for every pair $A, B \subseteq \Gamma$ of equal-size disjoint subsets, with $|A|,|B| \leq k$, the minimum edge-cut separating $A$ from $B$ in $G[C]$ has at least $\alpha_{\mathrm{BW}} \cdot|A|$ edges. Alternatively, we can send $|A|$ flow units from $A$ to $B$ inside $G[C]$ with edge congestion at most $1 / \alpha_{\mathrm{BW}}$. Notice that if $C$ does not have the $\left(k, \alpha_{\mathrm{BW}}\right)$-bandwidth property, then there is a partition $\left(C_{1}, C_{2}\right)$ of $C$, and two disjoint equal-size subsets $A \subseteq \Gamma \cap C_{1}, B \subseteq \Gamma \cap C_{2}$, with $|A|,|B| \leq k$, such that $\left|E_{G}\left(C_{1}, C_{2}\right)\right|<\alpha_{\mathrm{BW}} \cdot|A|$. We call such a partition a $\left(k, \alpha_{\mathrm{BW}}\right)$-violating cut of $C$. Even if we weaken the definition of the good routers and replace the $\alpha_{\mathrm{BW}}$-bandwidth property with the weaker $\left(k, \alpha_{\mathrm{BW}}\right)$-bandwidth property, we can still construct a good tree-of-sets system from a family of good routers, as the construction algorithm only uses the $\alpha_{\mathrm{BW}}$ bandwidth property of the routers in the weak sense by sending small amounts of flow (up to $k$ units) across the routers. Given the set $X_{i}^{\prime}$, we can now show that there is a

\footnotetext{
${ }^{6}$ The technical statement here is that if there is a small cut separating two large well-linked sets in a graph, then there is an edge that can be removed without affecting the well-linkedness of one of the sets.
} 
partition $\mathcal{W}_{i}$ of $X_{i}^{\prime}$ into clusters that have the $\left(k, \alpha_{\mathrm{BW}}\right)$-bandwidth property such that the number of edges connecting different clusters is bounded by $O\left(\left|\Gamma_{i}\right| / \ell_{0}\right)$.

Constructive proof. A constructive proof is more difficult for the following two reasons. First, given a large cluster $S_{i}$ that has the $\alpha_{\mathrm{BW}}$-bandwidth property but cannot send large amounts of flow to the terminals in $G$, we need an efficient algorithm for finding an edge that can be removed from $G\left[S_{i}\right]$ without violating the 1-well-linkedness of the terminals. Although we know that such an edge must exist, we do not have a constructive proof that allows us to find it. The second problem is related to the bandwidth-decomposition procedure. We know that, given $X_{i}^{\prime}$, there is a desired partition $\mathcal{W}_{i}$ of $X_{i}$ into clusters that have the $\left(k, \alpha_{\mathrm{BW}}\right)$-bandwidth property; however, we do not have an algorithmic version of this result. In particular, we need an efficient algorithm that finds a $\left(k, \alpha_{\mathrm{BW}}\right)$-violating cut in a cluster that does not have the $\left(k, \alpha_{\mathrm{BW}}\right)$ bandwidth property. (An efficient algorithm that gives a poly $(\log k)$ approximation by returning an $\left(\Omega(k), \alpha_{\mathrm{BW}} \cdot\right.$ poly $\left.\log k\right)$-violating cut would be sufficient, but as of now we do not have such an algorithm.)

In addition to a good clustering defined earlier, our algorithm uses a notion of acceptable clustering. An acceptable clustering is defined exactly like a good clustering, except large clusters are now allowed. Each small cluster in an acceptable clustering must have the $\alpha_{\mathrm{BW}}$-bandwidth property, and each large cluster must induce a connected graph in $G$.

To overcome the difficulties described previously, we define a potential function $\varphi$ over partitions $\mathcal{C}$ of $V(G)$. Given such a partition $\mathcal{C}, \varphi(\mathcal{C})$ is designed to be a good approximation of the number of edges connecting different clusters of $\mathcal{C}$. Additionally, $\varphi$ has the following two useful properties. If we are given an acceptable clustering $\mathcal{C}$, a large cluster $C \in \mathcal{C}$, and a $\left(k, \alpha_{\mathrm{BW}}\right)$-violating cut $\left(C_{1}, C_{2}\right)$ of $C$, then we can efficiently find a new acceptable clustering $\mathcal{C}^{\prime}$ with $\varphi\left(\mathcal{C}^{\prime}\right)<\varphi(\mathcal{C})-1 / n$. Similarly, if we are given an acceptable clustering $\mathcal{C}$, and a large cluster $C \in \mathcal{C}$, such that $C$ cannot send $w_{0} / 2$ flow units to the terminals, then we can efficiently find a new acceptable clustering $\mathcal{C}^{\prime}$ with $\varphi\left(\mathcal{C}^{\prime}\right)<\varphi(\mathcal{C})-1 / n$.

The algorithm consists of several phases. In every phase, we start with some good clustering $\mathcal{C}$, where in the first phase, $\mathcal{C}=\{\{v\} \mid v \in V(G)\}$. In each phase, we either find a good tree-of-sets system or a new good clustering $\mathcal{C}^{\prime}$, with $\varphi\left(\mathcal{C}^{\prime}\right) \leq \varphi(\mathcal{C})-1$. Therefore, after $O(|E(G)|)$ phases, we are guaranteed to find a good tree-of-sets system.

We now describe an execution of each phase. Let $\mathcal{C}$ be the current good clustering, and let $G^{\prime}$ be the corresponding legal contracted graph. As before, we find a partition $\left\{X_{1}, \ldots, X_{\ell_{0}}\right\}$ of $V\left(G^{\prime}\right) \backslash \mathcal{T}$, where for each $1 \leq i \leq \ell_{0}, \mid$ out $_{G^{\prime}}\left(X_{i}\right) \mid<O\left(\ell_{0}\left|E_{G^{\prime}}\left(X_{i}\right)\right|\right)$, using a simple randomized algorithm. For each set $X_{i}$, let $X_{i}^{\prime} \subseteq V(G) \backslash \mathcal{T}$ be the corresponding set of vertices in $G$, obtained by uncontracting each supernode $v_{C} \in X_{i}$. For each $1 \leq$ $i \leq \ell_{0}$, we also construct an acceptable clustering $\mathcal{C}_{i}$, containing all clusters $C \in \mathcal{C}$ with $C \cap X_{i}^{\prime}=\varnothing$, and all connected components of $G\left[X_{i}\right]$ (if any such connected component is a small cluster, we further partition it into clusters with $\alpha_{\mathrm{BW}}$-bandwidth property). We show that $\varphi\left(\mathcal{C}_{i}\right) \leq \varphi(\mathcal{C})-1$ for each $i$. We then perform several iterations.

In each iteration, we are given as input, for each $1 \leq i \leq \ell_{0}$, an acceptable clustering $\mathcal{C}_{i}$, with $\varphi\left(\mathcal{C}_{i}\right) \leq \varphi(\mathcal{C})-1$, where each large cluster of $\overline{\mathcal{C}}_{i}$ is contained in $X_{i}^{\prime}$. An iteration is executed as follows. If for some $1 \leq i \leq \ell_{0}$ the clustering $\mathcal{C}_{i}$ contains no large clusters, then $\mathcal{C}_{i}$ is a good clustering, with $\varphi\left(\mathcal{C}_{i}\right) \leq \varphi(\mathcal{C})-1$. We then finish the current phase and return the good clustering $\mathcal{C}_{i}$. Otherwise, for each $1 \leq i \leq \ell_{0}$, there is at least one large cluster $S_{i} \in \mathcal{C}_{i}$. We treat the clusters $\left\{S_{1}, \ldots, S_{\ell_{0}}\right\}$ as a potential good family of routers and try to construct a tree-of-sets system using them. If we succeed in building a good tree-of-sets system, then we are done, and we terminate the algorithm. Otherwise, we will obtain a certificate that one of the clusters $S_{i}$ is not a good router. The certificate 
is either a $\left(k, \alpha_{\mathrm{BW}}\right)$-violating partition of $S_{i}$ or a small cut (containing fewer than $w_{0} / 2$ edges), separating $S_{i}$ from the terminals. In either case, using the properties of the potential function, we can obtain a new acceptable clustering $\mathcal{C}_{i}^{\prime}$ with $\varphi\left(\mathcal{C}_{i}^{\prime}\right) \leq \varphi\left(\mathcal{C}_{i}\right)-1 / n$ to replace the current acceptable clustering $\mathcal{C}_{i}$. We then continue to the next iteration.

Overall, as long as we do not find a good tree-of-sets system, and do not find a good clustering $\mathcal{C}^{\prime}$ with $\varphi\left(\mathcal{C}^{\prime}\right) \leq \varphi(\mathcal{C})-1$, we make progress by lowering the potential of one of the acceptable clusterings $\mathcal{C}_{i}$ by at least $1 / n$. Therefore, after polynomially many iterations, we are guaranteed to complete the phase.

We note that Theorem 6 in Chuzhoy [2016] provides an algorithm that, given a cluster $X_{i}^{\prime}$, and an access to an oracle for computing $\left(k, \alpha_{\mathrm{BW}}\right)$-violating cuts, produces a partition $\mathcal{W}_{i}$ of $X_{i}^{\prime}$ into clusters that have the $\left(k, \alpha_{\mathrm{BW}}\right)$-bandwidth property, with the number of edges connecting different clusters suitably bounded. The bound on the number of edges is computed by using a charging scheme. The potential function that we use here, whose definition may appear nonintuitive, is modeled after this charging scheme.

In the following sections, we provide a formal proof of Theorem 4.3. We start by defining the different types of clusterings that we use and the potential function, and analyze its properties. We then turn to describe the algorithm itself.

\subsection{Vertex Clusterings and Legal Contracted Graphs}

Let $n=|V(G)|$. Our algorithm uses a parameter $\ell_{0}=\ell^{2}$. We use the following two parameters for the bandwidth property: $\alpha=\frac{1}{2^{11} \ell_{0} \log k}$, used to perform bandwidthdecomposition of clusters and $\alpha_{\mathrm{BW}}=\frac{\alpha}{\beta_{\mathrm{ARV}}(k)}=\Omega\left(\frac{1}{\ell^{2} \log ^{1.5} k}\right)$ - the value of the bandwidth parameter that we achieve. Finally, we use a parameter $w_{0}=\frac{k}{192 \ell_{0}^{3} \log k}$. We say that a cluster $C \subseteq V(G)$ is large if and only if $|\operatorname{out}(C)| \geq w_{0}$, and we say that it is small otherwise. From the statement of the theorem, we can assume that $w_{0}>\Delta$ and

$$
w=O\left(\frac{w_{0} \alpha^{2}}{\ell^{9} \Delta^{8} \log k}\right) .
$$

Next, we define acceptable and good vertex clusterings and legal contracted graphs, exactly as in Chuzhoy and Li [2016].

Definition 5.1. Given a partition $\mathcal{C}$ of the vertices of $V(G)$ into clusters, we say that $\mathcal{C}$ is an acceptable clustering of $G$ if and only if

—every terminal $t \in \mathcal{T}$ is in a separate cluster (i.e., $\{t\} \in \mathcal{C}$ );

-each small cluster $C \in \mathcal{C}$ has the the $\alpha_{\mathrm{BW}}$-bandwidth property; and

-for each large cluster $C \in \mathcal{C}, G[C]$ is connected.

An acceptable clustering that contains no large clusters is called a good clustering.

Definition 5.2. Given a good clustering $\mathcal{C}$ of $G$, a graph $H_{\mathcal{C}}$ is a legal contracted graph of $G$ associated with $\mathcal{C}$ if and only if we can obtain $H_{\mathcal{C}}$ from $G$ by contracting every $C \in \mathcal{C}$ into a supernode $v_{C}$. We remove all self-loops, but we do not remove parallel edges. (Note that the terminals are not contracted, as each terminal has its own cluster.)

Claim 5.3. If $G^{\prime}$ is a legal contracted graph for $G$, then $G^{\prime} \backslash \mathcal{T}$ contains at least $k / 3$ edges. 
Proof. For each terminal $t \in \mathcal{T}$, let $e_{t}$ be the unique edge adjacent to $t$ in $G^{\prime}$, and let $u_{t}$ be the other endpoint of $e_{t}$. We partition the terminals in $\mathcal{T}$ into groups, where two terminals $t, t^{\prime}$ belong to the same group if and only if $u_{t}=u_{t^{\prime}}$. Let $\mathcal{G}$ be the resulting partition of the terminals. Since the degree of every vertex in $G^{\prime}$ is at most $w_{0}$, each group $U \in \mathcal{G}$ contains at most $w_{0}$ terminals. Next, we partition the terminals in $\mathcal{T}$ into two subsets $X, Y$, where $|X|,|Y| \geq k / 3$, and for each group $U \in \mathcal{G}$, either $U \subseteq X$ or $U \subseteq Y$ holds. We can find such a partition by greedily processing each group $U \in \mathcal{G}$ and adding all terminals of $U$ to one of the subsets $X$ or $Y$ that currently contains fewer terminals. Finally, we remove terminals from set $X$ until $|X|=k / 3$, and we do the same for $Y$. Since the set $\mathcal{T}$ of terminals is node-well-linked in $G$, it is 1-edge-well-linked in $G^{\prime}$, so we can route $k / 3$ flow units from $X$ to $Y$ in $G^{\prime}$ with no edge congestion. Since no group $U$ is split between the two sets $X$ and $Y$, each flow path must contain at least one edge of $G^{\prime} \backslash \mathcal{T}$. Therefore, $\left|E\left(G^{\prime} \backslash \mathcal{T}\right)\right| \geq k / 3$.

Given a partition $\mathcal{C}$ of the vertices of $G$, we define a potential $\varphi(\mathcal{C})$ for this clustering, exactly as in Chuzhoy and $\mathrm{Li}[2016]$. The idea is that $\varphi(\mathcal{C})$ will serve as a tight bound on the number of edges connecting different clusters in $\mathcal{C}$. At the same time, the potential function is designed in such a way that we can perform several useful operations on the current clustering without increasing the potential.

Suppose that we are given any partition $\mathcal{C}$ of the vertices of $G$. We define $\varphi(\mathcal{C})$ as $\sum_{e \in E(G)} \varphi(\mathcal{C}, e)$, where $\varphi(\mathcal{C}, e)$ assigns a potential to each edge $e$; to avoid notational overload, we use $\varphi(e)$ for $\varphi(\mathcal{C}, e)$. If both endpoints of $e$ belong to the same cluster of $\mathcal{C}$, then we set its potential $\varphi(e)=0$. Otherwise, if $e=(u, v)$, and $u \in C$ with $|\operatorname{out}(C)|=z$, whereas $v \in C^{\prime}$ with $\left|\operatorname{out}\left(C^{\prime}\right)\right|=z^{\prime}$, then we set $\varphi(e)=1+\rho(z)+\rho\left(z^{\prime}\right)$, where $\rho$ is a nondecreasing real-valued function that we define in the following. We think of $\rho(z)$ as the contribution of $u$ and $\rho\left(z^{\prime}\right)$ as the contribution of $v$ to $\varphi(e)$. The function $\rho$ will be chosen to give a small (compared to 1) contribution to $\varphi(e)$ depending on the out-degree of the clusters that $e$ connects.

For an integer $z>0$, we define a potential $\rho(z)$ as follows. For $z<w_{0}, \rho(z)=4 \alpha \log z$. To define $\rho(z)$ for $z \geq w_{0}$, we consider the sequence $\left\{n_{0}, n_{1}, \ldots\right\}$ of numbers, where $n_{i}=\left(\frac{3}{2}\right)^{i} w_{0}$. The potentials for these numbers are $\rho\left(n_{0}\right)=\rho\left(w_{0}\right)=4 \alpha \log w_{0}+4 \alpha$, and for $i>0, \rho\left(n_{i}\right)=4 \frac{\alpha w_{0}}{n_{i}}+\rho\left(n_{i-1}\right)$. Notice that for all $i, \rho\left(n_{i}\right) \leq 12 \alpha+4 \alpha \log w_{0} \leq 8 \alpha \log w_{0} \leq$ $\frac{1}{2^{8} \ell_{0}}$. We now partition all integers $z>w_{0}$ into sets $Z_{1}, Z_{2}, \ldots$, where set $Z_{i}$ contains all integers $z$ with $n_{i-1} \leq z<n_{i}$. For $z \in Z_{i}$, we define $\rho(z)=\rho\left(n_{i-1}\right)$. This finishes the definition of $\rho$. Clearly, for all $z, \rho(z) \leq \frac{1}{2^{8} \ell_{0}}$.

ObSERvation 5.4. For a partition $\mathcal{C}$ of the vertices of $G$ and for an edge $e=(u, v) \in E(G)$, if $u, v$ belong to the same cluster of $\mathcal{C}$, then $\varphi(e)=0$. Otherwise, $1 \leq \varphi(e) \leq 1.1$.

Suppose that we are given a partition $\mathcal{C}$ of $V(G)$. The following theorem allows us to partition a small cluster $C$ into a collection of subclusters, each of which has the $\alpha_{\mathrm{BW}^{-}}$ bandwidth property, without increasing the overall potential. We call this procedure a bandwidth decomposition.

Theorem 5.5. Let $\mathcal{C}$ be a partition of $V(G)$, and let $C \in \mathcal{C}$ be any small cluster, such that $G[C]$ is connected. Then there is an efficient algorithm that finds a partition $\mathcal{W}$ of $C$ into small clusters, such that each cluster $R \in \mathcal{W}$ has the $\alpha_{\mathrm{BW}}$-bandwidth property, and additionally, if $\mathcal{C}^{\prime}$ is a partition obtained from $\mathcal{C}$ by removing $C$ and adding the clusters of $\mathcal{W}$ to it, then $\varphi\left(\mathcal{C}^{\prime}\right) \leq \varphi(\mathcal{C})$.

Proof. We maintain a partition $\mathcal{W}$ of $C$ into small clusters, where at the beginning, $\mathcal{W}=\{C\}$. We then perform several iterations. 
In each iteration, we select a cluster $S \in \mathcal{W}$ and set up the following instance of the sparsest cut problem. Let $\Gamma$ be the set of the interface vertices of $S$ in $G$. We then consider the graph $G[S]$, where the vertices of $\Gamma$ serve as terminals. We run the algorithm $\mathcal{A}_{\mathrm{ARV}}$ on the resulting instance of the sparsest cut problem. If the sparsity of the cut produced by the algorithm is less than $\alpha$, then we obtain a partition $(X, Y)$ of $S$, with $|E(X, Y)|<\alpha \cdot \min \{|\Gamma \cap X|,|\Gamma \cap Y|\} \leq \alpha \cdot \min \{|\operatorname{out}(S) \cap \operatorname{out}(X)|,|\operatorname{out}(S) \cap \operatorname{out}(Y)|\}$. In this case, we remove $S$ from $\mathcal{W}$ and add $X$ and $Y$ to $\mathcal{W}$ instead. Notice that since $S$ is a small cluster, $X$ and $Y$ are also small clusters. The algorithm ends when for every cluster $S \in \mathcal{W}$, algorithm $\mathcal{A}_{\mathrm{ARV}}$ returns a partition of sparsity at least $\alpha$. We are then guaranteed that every cluster in $\mathcal{W}$ has the $\alpha / \beta_{\mathrm{ARV}}(k)=\alpha_{\mathrm{BW}}$-bandwidth property, and it is easy to verify that all resulting clusters are small.

It now only remains to show that the potential does not increase. Each iteration of the algorithm is associated with a partition of the vertices of $G$, obtained from $\mathcal{C}$ by removing $C$ and adding all clusters of the current partition $\mathcal{W}$ of $C$ to it. It suffices to show that if $\mathcal{C}^{\prime}$ is the current partition of $V(G)$, and $\mathcal{C}^{\prime \prime}$ is the partition obtained after one iteration, where a set $S \in \mathcal{C}$ was replaced by two sets $X$ and $Y$, then $\varphi\left(\mathcal{C}^{\prime \prime}\right) \leq \varphi\left(\mathcal{C}^{\prime}\right)$.

Assume without loss of generality that $|\operatorname{out}(X)| \leq|\operatorname{out}(Y)|$, so $|\operatorname{out}(X)| \leq 2|\operatorname{out}(S)| / 3$. Let $z=|\operatorname{out}(S)|, z_{1}=|\operatorname{out}(X)|, z_{2}=|\operatorname{out}(Y)|$, and recall that $z, z_{1}, z_{2}<w_{0}$. The changes to the potential are the following:

- The potential of the edges in $\operatorname{out}(Y) \cap \operatorname{out}(S)$ only goes down.

-The potential of every edge in $\operatorname{out}(X) \cap \operatorname{out}(S)$ goes down by $\rho(z)-\rho\left(z_{1}\right)=4 \alpha \log z-$ $4 \alpha \log z_{1}=4 \alpha \log \frac{z}{z_{1}} \geq 4 \alpha \log 1.5 \geq 2.3 \alpha$, as $z_{1} \leq 2 z / 3$. Thus, the total decrease in the potential of the edges in $\operatorname{out}(X) \cap \operatorname{out}(S)$ is at least $2.3 \alpha \cdot|\operatorname{out}(X) \cap \operatorname{out}(S)|$.

-The edges in $E(X, Y)$ did not contribute to the potential initially and now contribute $1+\rho\left(z_{1}\right)+\rho\left(z_{2}\right) \leq 2$ each. Notice that $|E(X, Y)| \leq \alpha \cdot|\operatorname{out}(X) \cap \operatorname{out}(S)|$, and so they contribute at most $2 \alpha \cdot|\operatorname{out}(X) \cap \operatorname{out}(S)|$ in total.

Clearly, the overall potential decreases.

Assume that we are given an acceptable clustering $\mathcal{C}$ of $G$. We now define two operations on $G$, each of which produces a new acceptable clustering of $G$, whose potential is strictly smaller than $\varphi(\mathcal{C})$.

Action 1: Partitioning a large cluster. Suppose that we are given a large cluster $C$, and let $\Gamma$ be the interface of $C$ in $G$. We say that a partition $(X, Y)$ of $C$ is a $\left(w_{0}, \alpha\right)$-violating partition if and only if there are two subsets $\Gamma_{X} \subseteq \Gamma \cap X, \Gamma_{Y} \subseteq \Gamma \cap Y$ of vertices, with $\left|\Gamma_{X}\right|+\left|\Gamma_{Y}\right| \leq w_{0}$, and $|E(X, Y)|<\alpha \cdot \min \left\{\left|\Gamma_{X}\right|,\left|\Gamma_{Y}\right|\right\}$. Equivalently, $(X, Y)$ is an $\left(w_{0}, \alpha\right)$-violating partition if and only if $|E(X, Y)|<\alpha \cdot \min \left\{|\Gamma \cap X|,|\Gamma \cap Y|,\left\lfloor w_{0} / 2\right\rfloor\right\}$.

Suppose that we are given an acceptable clustering $\mathcal{C}$ of $G$, a large cluster $C \in \mathcal{C}$, and an $\left(w_{0}, \alpha\right)$-violating partition $(X, Y)$ of $C$. To perform this operation, we first replace $C$ with $X$ and $Y$ in $\mathcal{C}$. If, additionally, either of the clusters, $X$ or $Y$, becomes small, we perform a bandwidth decomposition of that cluster using Theorem 5.5 and update $\mathcal{C}$ with the resulting partition. Clearly, the final partitioning $\mathcal{C}^{\prime}$ is an acceptable clustering. We denote this operation by PARTITION $(C, X, Y)$.

Claim 5.6. Let $\mathcal{C}^{\prime}$ be the outcome of operation PARTITION $(C, X, Y)$. Then $\varphi\left(\mathcal{C}^{\prime}\right)<$ $\varphi(\mathcal{C})-1 / n$.

Proof. Let $\mathcal{C}^{\prime \prime}$ be the clustering obtained from $\mathcal{C}$ by replacing $C$ with $X$ and $Y$. From Theorem 5.5, it is sufficient to prove that $\varphi\left(\mathcal{C}^{\prime \prime}\right)<\varphi(\mathcal{C})-1 / n$.

Assume without loss of generality that $|\operatorname{out}(X)| \leq|\operatorname{out}(Y)|$. Let $z=|\operatorname{out}(C)|, z_{1}=$ $|\operatorname{out}(X)|, z_{2}=|\operatorname{out}(Y)|$, so $z_{1}<2 z / 3$. Assume that $z \in Z_{i}$. Then either $z_{1} \in Z_{i^{\prime}}$ for $i^{\prime} \leq i-1$ or $z_{1}<w_{0}$. The potential of the edges in out $(Y) \cap \operatorname{out}(C)$ does not increase. 
The only other changes in the potential are the following: the potential of each edge in $\operatorname{out}(X) \cap \operatorname{out}(C)$ decreases by $\rho(z)-\rho\left(z_{1}\right)$, and the potential of every edge in $E(X, Y)$ increases from 0 to at most 1.1 . We consider two cases.

First, if $z_{1}<w_{0}$, then $\rho(z) \geq 4 \alpha+\rho\left(z_{1}\right)$. Thus, the potential of each edge in $\operatorname{out}(X) \cap$ out $(C)$ decreases by at least $4 \alpha$, and the overall decrease in potential due to these edges is at least $4 \alpha|\operatorname{out}(X) \cap \operatorname{out}(C)|$. The total increase in potential due to the edges in $E(X, Y)$ is bounded by $1.1|E(X, Y)|<1.1 \alpha\left|\Gamma_{X}\right| \leq 1.1 \alpha \mid$ out $(X) \cap$ out $(C) \mid$, so the overall potential decreases by at least $2 \alpha|\operatorname{out}(X) \cap \operatorname{out}(C)|>1 / n$.

The second case is when $z_{1} \geq w_{0}$. Assume that $z_{1} \in Z_{i^{\prime}}$. Then $n_{i^{\prime}} \leq 3 z_{1} / 2$, and since $i^{\prime} \leq i-1$ must hold, $\rho(z) \geq \frac{4 \alpha w_{0}}{n_{i^{\prime}}}+\rho\left(n_{i^{\prime}-1}\right)=\frac{4 \alpha w_{0}}{n_{i^{\prime}}}+\rho\left(z_{1}\right) \geq \frac{8 \alpha w_{0}}{3 z_{1}}+\rho\left(z_{1}\right)$. Thus, the potential of each edge in $\operatorname{out}(X) \cap \operatorname{out}(C)$ decreases by at least $\frac{8 \alpha w_{0}}{3 z_{1}}$, and the total decrease in potential due to these edges is at least $\frac{8 \alpha w_{0}}{3 z_{1}} \cdot|\operatorname{out}(X) \cap \operatorname{out}(C)| \geq \frac{4 \alpha w_{0}}{3}$, as $|\operatorname{out}(X) \cap \operatorname{out}(C)| \geq z_{1} / 2$. The total increase in the potential due to the edges in $E(X, Y)$ is bounded by $1.1|E(X, Y)|<0.55 \alpha w_{0}$, as $|E(X, Y)| \leq \alpha w_{0} / 2$. Overall, the total potential decreases by at least $\frac{2 \alpha w_{0}}{3}>1 / n$.

Action 2: Separating a large cluster. Let $\mathcal{C}$ be an acceptable clustering, and let $C \in \mathcal{C}$ be a large cluster in $\mathcal{C}$. Assume further that we are given a partition $(A, B)$ of $V(G)$ with $C \subseteq A, \mathcal{T} \subseteq B$ and $\left|E_{G}(A, B)\right|<w_{0} / 2$. We perform the following operation, which we denote by $\operatorname{SEPARATE}(C, A)$.

Consider a cluster $S \in \mathcal{C}$. If $S \backslash A \neq \emptyset$ and $|\operatorname{out}(S \backslash A)|>|\operatorname{out}(S)|$, we modify $A$ by removing all vertices of $S$ from it. Notice that in this case, the number of edges in $E(S)$ that originally contributed to the cut $(A, B),|E(S \cap A, S \cap B)|>|\operatorname{out}(S) \cap E(A)|$ must hold, so $|\operatorname{out}(A)|$ only goes down as a result of this modification. We assume from now on that if $|S \backslash A| \neq \emptyset$, then $|\operatorname{out}(S \backslash A)| \leq|\operatorname{out}(S)|$. In particular, if $S$ is a small cluster, and $S \backslash A \neq \emptyset$, then $S \backslash A$ is also a small cluster.

We build a new partition $\mathcal{C}^{\prime}$ of $V(G)$ as follows. First, we add every connected component of $G[A]$ to $\mathcal{C}^{\prime}$. Notice that all of these clusters are small, as $|\operatorname{out}(A)|<w_{0} / 2$. Next, for every cluster $S \in \mathcal{C}$, such that $S \backslash A \neq \emptyset$, we add every connected component of $G[S \backslash A]$ to $\mathcal{C}^{\prime}$. Notice that every terminal $t \in \mathcal{T}$ is added as a separate cluster to $\mathcal{C}^{\prime}$. So far, we have defined a new partition $\mathcal{C}^{\prime}$ of $V(G)$. This partition may not be acceptable, as we are not guaranteed that every small cluster of $\mathcal{C}^{\prime}$ has the bandwidth property. In our final step, we perform the bandwidth decomposition of every small cluster of $\mathcal{C}^{\prime}$, using Theorem 5.5, and obtain the final acceptable partition $\mathcal{C}^{\prime \prime}$ of vertices of $G$. Notice that if $S \in \mathcal{C}^{\prime \prime}$ is a large cluster, then there must be some large cluster $S^{\prime}$ in the original partition $\mathcal{C}$ with $S \subseteq S^{\prime}$.

\section{Claim 5.7. Let $\mathcal{C}^{\prime \prime}$ be the outcome of operation $\operatorname{SEPARATE}(C, A)$. Then $\varphi\left(\mathcal{C}^{\prime \prime}\right) \leq \varphi(\mathcal{C})-1$.}

Proof. To prove the claim, it is enough to prove that $\varphi\left(\mathcal{C}^{\prime}\right) \leq \varphi(\mathcal{C})-1$, because from Theorem 5.5, bandwidth decompositions of small clusters do not increase the potential.

We now show that $\varphi\left(\mathcal{C}^{\prime}\right) \leq \varphi(\mathcal{C})-1$. We can bound the changes in the potential as follows:

-Every edge in out $(A)$ contributes at most 1.1 to the potential of $\mathcal{C}^{\prime \prime}$, and there are at most $\frac{w_{0}-1}{2}$ such edges.

-Every edge in out $(C)$ contributes at least 1 to the potential of $\mathcal{C}^{\prime}$, and there are at least $w_{0}$ such edges, since $C$ is a large cluster.

For every other edge $e$, the potential of $e$ does not increase. Indeed, let $e=(u, v)$, where $u \in S_{1}, v \in S_{2}$, with $S_{1}, S_{2} \in \mathcal{C}^{\prime}$ and $S_{1}, S_{2} \nsubseteq A$. Then there are clusters $S_{1}^{\prime}, S_{2}^{\prime} \in \mathcal{C}$, with $S_{1} \subseteq S_{1}^{\prime}$ and $S_{2} \subseteq S_{2}^{\prime}$. Notice that $S_{1}^{\prime} \neq S_{2}^{\prime}$, as $S_{1}$ and $S_{2}$ correspond to connected 
components of $S_{1}^{\prime}$ and $S_{2}^{\prime}$, respectively, and therefore no edge can connect them. From our construction of $\mathcal{C}^{\prime},\left|\operatorname{out}\left(S_{1}\right)\right| \leq\left|\operatorname{out}\left(S_{1}^{\prime}\right)\right|$ and $\left|\operatorname{out}\left(S_{2}\right)\right| \leq\left|\operatorname{out}\left(S_{2}^{\prime}\right)\right|$, so the potential of $e$ cannot increase. Thus, the decrease in the potential is at least $w_{0}-\frac{1.1\left(w_{0}-1\right)}{2} \geq 1$.

To summarize, given an acceptable clustering $\mathcal{C}$ of the vertices of $G$, let $E^{\prime}$ be the set of edges whose endpoints belong to distinct clusters of $\mathcal{C}$. Then $\left|E^{\prime}\right| \leq \varphi(\mathcal{C}) \leq 1.1\left|E^{\prime}\right|$. Thus, the potential is a good estimate on the number of edges connecting the different clusters. We have also defined two actions on large clusters of $\mathcal{C}$ : PARTITION $(C, X, Y)$ can be performed if we are given a large cluster $C \in \mathcal{C}$ and a $\left(w_{0}, \alpha\right)$-violating partition $(X, Y)$ of $C$, and SEPARATE $(C, A)$, where $(A, V(G) \backslash A)$ is a partition of $V(G)$ with $|\operatorname{out}(A)|<w_{0} / 2$, separating a large cluster $C$ from the terminals. Each such action returns a new acceptable clustering, whose potential goes down by at least $1 / n$. Both operations ensure that if $S$ is a large cluster in the new clustering, then there is some large cluster $S^{\prime}$ in the original clustering with $S \subseteq S^{\prime}$.

\subsection{The Algorithm}

We maintain, throughout the algorithm, a good clustering $\mathcal{C}$ of $G$. Initially, $\mathcal{C}$ is a partition of $V(G)$, where every vertex of $G$ belongs to a distinct cluster-that is, $\mathcal{C}=\{\{v\} \mid v \in V(G)\}$. Clearly, this is a good clustering, as $\Delta<w_{0}$. The algorithm consists of several phases. In every phase, we start with some good clustering $\mathcal{C}$ and the corresponding legal contracted graph $H_{\mathcal{C}}$. The phase output is either a good tree-of-sets system or another good clustering $\mathcal{C}^{\prime}$ such that $\varphi\left(\mathcal{C}^{\prime}\right) \leq \varphi(\mathcal{C})-1$. In the former case, we terminate the algorithm and output the tree-of-sets system. In the latter case, we continue to the next phase. After $O(|E(G)|)$ phases, our algorithm will then successfully terminate with a good tree-of-sets system. It is therefore enough to prove the following theorem.

THEOREM 5.8. Let $\mathcal{C}$ be a good clustering of the vertices of $G$, and let $H_{\mathcal{C}}$ be the corresponding legal contracted graph. Then there is an efficient randomized algorithm that with high probability either computes a good tree-of-sets system or finds a new good clustering $\mathcal{C}^{\prime}$ such that $\varphi\left(\mathcal{C}^{\prime}\right) \leq \varphi(\mathcal{C})-1$.

The rest of this section is dedicated to proving Theorem 5.8. We assume that we are given a good clustering $\mathcal{C}$ of the vertices of $G$ and the corresponding legal contracted graph $G^{\prime}=H_{\mathcal{C}}$.

Let $m=\left|E\left(G^{\prime} \backslash \mathcal{T}\right)\right|$. From Claim $5.3, m \geq k / 3$. As a first step, we randomly partition the vertices in $G^{\prime} \backslash \mathcal{T}$ into $\ell_{0}$ subsets $X_{1}, \ldots, X_{\ell_{0}}$, where each vertex $v \in V\left(G^{\prime}\right) \backslash \mathcal{T}$ selects an index $1 \leq j \leq \ell_{0}$ independently uniformly at random and is then added to $X_{j}$. We need the following claim.

CLAIM 5.9. With probability at least $\frac{1}{2}$, for each $1 \leq j \leq \ell_{0},\left|\operatorname{out}_{G^{\prime}}\left(X_{j}\right)\right|<\frac{10 \mathrm{~m}}{\ell_{0}}$, whereas $\left|E_{G^{\prime}}\left(X_{j}\right)\right| \geq \frac{m}{2 \ell_{0}^{2}}$.

Proof. Let $H=G^{\prime} \backslash \mathcal{T}$. Fix some $1 \leq j \leq \ell_{0}$. Let $\mathcal{E}_{1}(j)$ be the bad event that $\sum_{v \in X_{j}} d_{H}(v)>\frac{2 m}{\ell_{0}} \cdot\left(1+\frac{1}{\ell_{0}}\right)$. To bound the probability of $\mathcal{E}_{1}(j)$, we define, for each vertex $v \in V(H)$, a random variable $x_{v}$, whose value is $\frac{d_{H}(v)}{w_{0}}$ if $v \in X_{j}$ and 0 otherwise. Notice that $x_{v} \in[0,1]$, and the random variables $\left\{x_{v}\right\}_{v \in V(H)}$ are pairwise independent. Let $B=\sum_{v \in V(H)} x_{v}$. Then the expectation of $B, \mu_{1}=\sum_{v \in V(H)} \frac{d_{H}(v)}{\ell_{0} w_{0}}=\frac{2 m}{\ell_{0} w_{0}}$. Using the standard Chernoff bound (e.g., see Theorem 1.1 in Dubhashi and Panconesi [2009]),

$$
\operatorname{Pr}\left[\mathcal{E}_{1}(j)\right]=\operatorname{Pr}\left[B>\left(1+1 / \ell_{0}\right) \mu_{1}\right] \leq e^{-\mu_{1} /\left(3 \ell_{0}^{2}\right)}=e^{-\frac{2 m}{3 \ell_{0}^{3} w_{0}}}<\frac{1}{6 \ell_{0}}
$$

since $m \geq k / 3$ and $w_{0}=\frac{k}{192 \ell_{0}^{3} \log k}$. 
For each terminal $t \in \mathcal{T}$, let $e_{t}$ be the unique edge adjacent to $t$ in graph $G^{\prime}$, and let $u_{t}$ be its other endpoint. Let $U=\left\{u_{t} \mid t \in \mathcal{T}\right\}$. For each vertex $u \in U$, let $w(u)$ be the number of terminals $t$ such that $u=u_{t}$. Notice that $w(u) \leq w_{0}$ must hold. We say that a bad event $\mathcal{E}_{2}(j)$ happens if and only if $\sum_{u \in U \cap X_{j}} w(u) \geq \frac{k}{\ell_{0}} \cdot\left(1+\frac{1}{\ell_{0}}\right)$. To bound the probability of the event $\mathcal{E}_{2}(j)$, we define, for each $u \in U$, a random variable $y_{u}$, whose value is $w(u) / w_{0}$ if and only if $u \in X_{j}$, and is 0 otherwise. Notice that $y_{u} \in[0,1]$, and the variables $y_{u}$ are independent for all $u \in U$. Let $Y=\sum_{u \in U} y_{u}$. The expectation of $Y$ is $\mu_{2}=\frac{k}{w_{0} \ell_{0}}$, and event $\mathcal{E}_{2}(j)$ holds if and only if $Y \geq \frac{k}{w_{0} \ell_{0}} \cdot\left(1+\frac{1}{\ell_{0}}\right) \geq \mu_{2} \cdot\left(1+\frac{1}{\ell_{0}}\right)$. Using the standard Chernoff bound again, we get that

$$
\operatorname{Pr}\left[\mathcal{E}_{2}(j)\right] \leq e^{-\mu_{2} /\left(3 \ell_{0}^{2}\right)} \leq e^{-k /\left(3 w_{0} \ell_{0}^{3}\right)} \leq \frac{1}{6 \ell_{0}}
$$

since $w_{0}=\frac{k}{192 \ell_{0}^{3} \log k}$. Notice that if events $\mathcal{E}_{1}(j), \mathcal{E}_{2}(j)$ do not hold, then

$$
\left|\operatorname{out}_{G^{\prime}}\left(X_{j}\right)\right| \leq \sum_{v \in X_{j}} d_{H}(v)+\sum_{u \in U \cap X_{j}} w(u) \leq\left(1+\frac{1}{\ell_{0}}\right)\left(\frac{2 m}{\ell_{0}}+\frac{k}{\ell_{0}}\right)<\frac{10 m}{\ell_{0}}
$$

since $m \geq k / 3$.

Let $\mathcal{E}_{3}(j)$ be the bad event that $\left|E_{G^{\prime}}\left(X_{j}\right)\right|<\frac{m}{2 \ell_{0}^{2}}$. We next prove that $\operatorname{Pr}\left[\mathcal{E}_{3}(j)\right] \leq \frac{1}{6 \ell_{0}}$. We say that two edges $e, e^{\prime} \in E\left(G^{\prime} \backslash \mathcal{T}\right)$ are independent if and only if they do not share endpoints. Our first step is to compute a partition $U_{1}, \ldots, U_{z}$ of the set $E\left(G^{\prime} \backslash \mathcal{T}\right)$ of edges, where $z \leq 2 w_{0}$, such that for each $1 \leq i \leq z,\left|U_{i}\right| \geq \frac{m}{4 w_{0}}$, and all edges in set $U_{i}$ are mutually independent. To compute such a partition, we construct an auxiliary graph $Z$, whose vertex set is $\left\{v_{e} \mid e \in E(H)\right\}$, and there is an edge $\left(v_{e}, v_{e^{\prime}}\right)$ if and only if $e$ and $e^{\prime}$ are not independent. Since the maximum vertex degree in $G^{\prime}$ is at most $w_{0}$, the maximum vertex degree in $Z$ is bounded by $2 w_{0}-2$. Using the Hajnal-Szemerédi theorem [Hajnal and Szemerédi 1970], we can find a partition $V_{1}, \ldots, V_{z}$ of the vertices of $Z$ into $z \leq 2 w_{0}$ subsets, where each subset $V_{i}$ is an independent set, and $\left|V_{i}\right| \geq \frac{|V(Z)|}{z}-1 \geq \frac{m}{4 w_{0}}$. The partition $V_{1}, \ldots, V_{z}$ of the vertices of $Z$ gives the desired partition $U_{1}, \ldots, U_{z}$ of the edges of $G^{\prime} \backslash \mathcal{T}$. For each $1 \leq i \leq r$, we say that the bad event $\mathcal{E}_{3}^{i}(j)$ happens if and only if $\left|U_{i} \cap E\left(X_{j}\right)\right|<\frac{\left|U_{i}\right|}{2 \ell_{0}^{2}}$. Notice that if $\mathcal{E}_{3}(j)$ happens, then event $\mathcal{E}_{3}^{i}(j)$ must happen for some $1 \leq i \leq z$. Fix some $1 \leq i \leq z$. The expectation of $\left|U_{i} \cap E\left(X_{j}\right)\right|$ is $\mu_{3}=\frac{\left|U_{i}\right|}{\ell_{0}^{2}}$. Since all edges in $U_{i}$ are independent, we can use the standard Chernoff bound to bound the probability of $\mathcal{E}_{3}^{i}(j)$ as follows:

$$
\operatorname{Pr}\left[\mathcal{E}_{3}^{i}(j)\right]=\operatorname{Pr}\left[\left|U_{i} \cap E\left(X_{j}\right)\right|<\mu_{3} / 2\right] \leq e^{-\mu_{3} / 8}=e^{-\frac{\left|U_{i}\right|}{8 \ell_{0}^{2}}} .
$$

Since $\left|U_{i}\right| \geq \frac{m}{4 w_{0}}, m \geq k / 3, w_{0}=\frac{k}{192 \ell_{0}^{3} \log k}$, this is bounded by $\frac{1}{k^{2}} \leq \frac{1}{12 w_{0} \ell_{0}}$. We conclude that $\operatorname{Pr}\left[\mathcal{E}_{3}^{i}(j)\right] \leq \frac{1}{12 w_{0} \ell_{0}}$, and by using the union bound over all $1 \leq i \leq z, \operatorname{Pr}\left[\mathcal{E}_{3}(j)\right] \leq \frac{1}{6 \ell_{0}}$.

Using the union bound over all $1 \leq j \leq \ell_{0}$, with probability at least $\frac{1}{2}$, none of the events $\mathcal{E}_{1}(j), \mathcal{E}_{2}(j), \mathcal{E}_{3}(j)$ for $1 \leq j \leq \ell_{0}$ happen, and so for each $1 \leq j \leq \ell_{0}$, $\left|\operatorname{out}_{G^{\prime}}\left(X_{j}\right)\right|<\frac{10 m}{\ell_{0}}$ and $\left|E_{G^{\prime}}\left(X_{j}\right)\right| \geq \frac{m}{2 \ell_{0}^{2}}$.

Given a partition $X_{1}, \ldots, X_{\ell_{0}}$, we can efficiently check whether the conditions of Claim 5.9 hold. If they do not hold, we repeat the randomized partitioning procedure. From Claim 5.9, we are guaranteed that with high probability, after poly $(n)$ iterations, 
we will obtain a partition with the desired properties. Assume now that we are given the partition $X_{1}, \ldots, X_{\ell_{0}}$ of $V\left(G^{\prime}\right) \backslash \mathcal{T}$, for which the conditions of Claim 5.9 hold. Then for each $1 \leq j \leq \ell_{0},\left|E_{G^{\prime}}\left(X_{j}\right)\right|>\frac{\mid \text { out }_{G^{\prime}}\left(X_{j}\right) \mid}{20 \ell_{0}}$. Let $X_{j}^{\prime} \subseteq V(G) \backslash \mathcal{T}$ be the set obtained from $X_{j}$, after we uncontract each cluster-that is, for each supernode $v_{C} \in X_{j}$, we replace $v_{C}$ with the vertices of $C$. Notice that $\left\{X_{j}^{\prime}\right\}_{j=1}^{\ell_{0}}$ is a partition of $V(G) \backslash \mathcal{T}$.

The plan for the rest of the proof is as follows. For each $1 \leq j \leq \ell_{0}$, we will maintain an acceptable clustering $\mathcal{C}_{j}$ of the vertices of $G$. In other words, for each $1 \leq j \leq \ell_{0}$, $\mathcal{C}_{j}$ is a partition of $V(G)$. In addition to being an acceptable clustering, it will have the following property:

(P1) If $C \in \mathcal{C}_{j}$ is a large cluster, then $C \subseteq X_{j}^{\prime}$.

The initial partition $\mathcal{C}_{j}$ for $1 \leq j \leq \ell_{0}$ is obtained as follows. Recall that $\mathcal{C}$ is the current good clustering of the vertices of $G$, and every cluster $C \in \mathcal{C}$ is either contained in $X_{j}^{\prime}$ or disjoint from it. First, we add to $\mathcal{C}_{j}$ all clusters $C \in \mathcal{C}$ with $C \cap X_{j}^{\prime}=\emptyset$. Next, we add to $\mathcal{C}_{j}$ all connected components of $G\left[X_{j}^{\prime}\right]$. If any of these components is a small cluster, we then perform the bandwidth decomposition of this cluster using Theorem 5.5 and update $\mathcal{C}_{j}$ accordingly. Let $\mathcal{C}_{j}$ be the resulting final partition. Clearly, it is an acceptable clustering with property (P1). Moreover, the following claim shows that $\varphi\left(\mathcal{C}_{j}\right) \leq \varphi(\mathcal{C})-1$.

Claim 5.10. For each $1 \leq j \leq \ell_{0}, \varphi\left(\mathcal{C}_{j}\right) \leq \varphi(\mathcal{C})-1$.

Proof. Let $\mathcal{C}_{j}^{\prime}$ be the partition of $V(G)$, obtained as follows: we add to $\mathcal{C}_{j}^{\prime}$ all clusters $C \in \mathcal{C}$ with $C \cap X_{j}^{\prime}=\emptyset$, and we add all connected components of $G\left[X_{j}^{\prime}\right]$ to $\mathcal{C}_{j}^{\prime}$ (i.e., $\mathcal{C}_{j}^{\prime}$ is obtained like $\mathcal{C}_{j}$, except we do not perform the bandwidth decompositions of the small clusters). From Theorem 5.5, it is enough to prove that $\varphi\left(\mathcal{C}_{j}^{\prime}\right) \leq \varphi(\mathcal{C})-1$. The changes of the potential from $\mathcal{C}$ to $\mathcal{C}_{j}^{\prime}$ can be bounded as follows:

-The edges in $E_{G^{\prime}}\left(X_{j}\right)$ contribute at least 1 to $\varphi(\mathcal{C})$ and contribute 0 to $\varphi\left(\mathcal{C}_{j}^{\prime}\right)$.

-The potential of the edges in $\operatorname{out}_{G}\left(X_{j}^{\prime}\right)$ may increase. The increase is at most $\rho(n) \leq \frac{1}{2^{8} \ell_{0}}$ per edge. Thus, the total increase is at most $\frac{\mid \text { out }_{G^{\prime}}\left(X_{j}\right) \mid}{2^{8} \ell_{0}} \leq \frac{\left|E_{G^{\prime}}\left(X_{j}\right)\right|}{4}$. These are the only edges whose potential may increase.

Overall, the decrease in the potential is at least $\frac{\left|E_{G^{\prime}}\left(X_{j}\right)\right|}{2} \geq \frac{m}{4 \ell_{0}^{2}} \geq \frac{k}{12 \ell_{0}^{2}} \geq 1$.

If any of the partitions $\mathcal{C}_{1}, \ldots, \mathcal{C}_{\ell_{0}}$ is a good clustering, then we have found a good clustering $\mathcal{C}^{\prime}$ with $\varphi\left(\mathcal{C}^{\prime}\right) \leq \varphi(\mathcal{C})-1$. We terminate the algorithm and return $\mathcal{C}^{\prime}$. Otherwise, we select an arbitrary large cluster $S_{j} \in \mathcal{C}_{j}$ for each $j$. We then consider the resulting collection $S_{1}, \ldots, S_{\ell_{0}}$ of large clusters and try to exploit them to construct a good treeof-sets system. Since for each $1 \leq j \leq \ell_{0}, S_{j} \subseteq X_{j}^{\prime}$, the sets $S_{1}, \ldots, S_{\ell_{0}}$ are mutually disjoint and do not contain terminals. Our algorithm performs several iterations using the following theorem.

THEOREM 5.11. There is an efficient randomized algorithm that, given a collection $\left\{S_{1}, \ldots, S_{\ell_{0}}\right\}$ of disjoint vertex subsets of $G$, where for all $1 \leq j \leq \ell_{0}, S_{j} \cap \mathcal{T}=\emptyset$, with high probability computes one of the following:

—a good tree-of-sets system in $G$; or

- $a\left(w_{0}, \alpha\right)$-violating partition $(X, Y)$ of $S_{j}$, for some $1 \leq j \leq \ell_{0}$; or

- a partition $(A, B)$ of $V(G)$ with $S_{j} \subseteq A, \mathcal{T} \subseteq B$ and $\left|E_{G}(A, B)\right|<w_{0} / 2$, for some $1 \leq j \leq \ell_{0}$. 
We provide the proof of Theorem 5.11 in the following section and complete the proof of Theorem 5.8 here. Suppose that we are given a good clustering $\mathcal{C}$ of the vertices of $G$. For each $1 \leq j \leq \ell_{0}$, we compute an acceptable clustering $\mathcal{C}_{j}$ of $V(G)$ as described earlier. If any of the partitions $\mathcal{C}_{j}$ is a good clustering, we will terminate the algorithm and return $\mathcal{C}_{j}$. From the preceding discussion, $\varphi\left(\mathcal{C}_{j}\right) \leq \varphi(\mathcal{C})-1$. Otherwise, for each $1 \leq j \leq \ell_{0}$, we select any large cluster $S_{j} \in \mathcal{C}_{j}$ and apply Theorem 5.11 to the current family $\left\{S_{1}, \ldots, S_{\ell_{0}}\right\}$ of large clusters. If the outcome of Theorem 5.11 is a good tree-ofsets system, we will terminate the algorithm and return this tree-of-sets system, and we say that the iteration is successful. Otherwise, we apply the appropriate action: $\operatorname{PARTITION}\left(S_{j}, X, Y\right)$ or SEPARATE $\left(S_{j}, A\right)$ to the clustering $\mathcal{C}_{j}$. As a result, we obtain an acceptable clustering $\mathcal{C}_{j}^{\prime}$ with $\varphi\left(\mathcal{C}_{j}^{\prime}\right) \leq \varphi\left(\mathcal{C}_{j}\right)-1 / n$. Moreover, it is easy to see that this clustering also has Property (P1): if the PARTITION operation is performed, then we only partition $S_{j}$; if the SEPARATE operation is performed, then for every large cluster $S$ in the new partition $\mathcal{C}_{j}^{\prime}$, there is a large cluster $S^{\prime} \in \mathcal{C}_{j}$ with $S \subseteq S^{\prime}$.

If all clusters in $\mathcal{C}_{j}^{\prime}$ are small, then we can again terminate the algorithm with a $\operatorname{good}$ clustering $\mathcal{C}_{j}^{\prime}$, with $\varphi\left(\mathcal{C}_{j}^{\prime}\right) \leq \varphi\left(\mathcal{C}_{j}\right)-1 / n \leq \varphi(\mathcal{C})-1-1 / n$ (recall that Claim 5.10 shows that $\left.\varphi\left(\mathcal{C}_{j}\right) \leq \varphi(\mathcal{C})-1\right)$. Otherwise, we select an arbitrary large cluster $S_{j}^{\prime} \in \mathcal{C}_{j}^{\prime}$ and continue to the next iteration. Overall, as long as we do not complete a successful iteration, and we do not find a good clustering $\mathcal{C}^{\prime}$ of $V(G)$ with $\varphi\left(\mathcal{C}^{\prime}\right) \leq \varphi(\mathcal{C})-1$, we make progress in each iteration by decreasing the potential of one of the partitions $\mathcal{C}_{j}$ by at least $1 / n$ by performing either a SEPARATE or a PARTITION operation on one of the large clusters of $\mathcal{C}_{j}$. After at most $1.1|E(G)| \cdot n \cdot \ell_{0}$ iterations, we are then guaranteed to complete a successful iteration, or find a good clustering $\mathcal{C}^{\prime}$ with $\varphi\left(\mathcal{C}^{\prime}\right) \leq \varphi(\mathcal{C})-1$, and finish the algorithm. Therefore, to complete the proof of Theorem 5.8, it is now enough to prove Theorem 5.11.

\subsection{Proof of Theorem 5.11}

Let $\mathcal{R}=\left\{S_{1}, \ldots, S_{\ell_{0}}\right\}$. We start by checking that for each $1 \leq j \leq \ell_{0}$, the vertices of $S_{j}$ can send $w_{0} / 2$ flow units in $G$ to the terminals with no edge congestion. If this is not the case for some set $S_{j}$, then there is a partition $(A, B)$ of $V(G)$ with $S_{j} \subseteq A, \mathcal{T} \subseteq B$ and $\left|E_{G}(A, B)\right|<w_{0} / 2$. We then return the partition $(A, B)$ of $V(G)$ and finish the algorithm. From now on, each set $S_{j}$ can send $w_{0} / 2$ flow units in $G$ to the terminals with no edge congestion. We assume without loss of generality that this flow is integral.

Since the set $\mathcal{T}$ of terminals is node-well-linked, every pair $\left(S_{j}, S_{j^{\prime}}\right)$ of vertex subsets from $\mathcal{R}$ can send $w_{0} / 2$ flow units to each other with edge congestion at most 3: concatenate the flows from $S_{j}$ to a subset $\mathcal{T}_{1}$ of the terminals, from $S_{j^{\prime}}$ to a subset $\mathcal{T}_{2}$ of the terminals, and the flow between the two subsets of the terminals. Scaling this flow down by factor $3 \Delta$ and using the integrality of flow, for each such pair $\left(S_{j}, S_{j^{\prime}}\right)$, there are at least $\left\lfloor\frac{w_{0}}{6 \Delta}\right\rfloor$ node-disjoint paths connecting $S_{j}$ to $S_{j^{\prime}}$ in $G$. We can assume that these paths do not contain terminals, as the degree of every terminal in $G$ is 1 .

The algorithm consists of two phases. In the first phase, we attempt to construct a tree-of-sets system using the collection $\mathcal{R}$ of vertex subsets. If we fail to do so, we will either return an $\left(w_{0}, \alpha\right)$-violating cut in some cluster $S_{j} \in \mathcal{R}$ or will identify a subset $\mathcal{R}^{\prime} \subseteq \mathcal{R}$ of $\ell$ clusters, and for each cluster $S_{j} \in \mathcal{R}^{\prime}$, a large subset $E_{j} \subseteq \operatorname{out}\left(S_{j}\right)$ of edges, such that for each $S_{i}, S_{j} \in \mathcal{R}^{\prime}$, there are many disjoint paths connecting the edges in $E_{j}$ to the edges in $E_{i}$ in $G$. In the second phase, we exploit the clusters in $\mathcal{R}^{\prime}$ to build the tree-of-sets system.

Given a graph $\mathbf{G}$ and a subset $\tilde{\mathcal{T}}$ of vertices called terminals, we say that a pair $\left(t, t^{\prime}\right)$ of terminals is $\lambda$-edge connected if and only if there are at least $\lambda$ paths connecting $t$ to $t^{\prime}$ in $\mathbf{G}$ that are mutually edge disjoint. Let $\lambda\left(t, t^{\prime}\right)$ be the largest value $\lambda$ such that $t$ and $t^{\prime}$ are $\lambda$-edge connected, and let $\lambda_{\mathbf{G}}(\tilde{\mathcal{T}})=\min _{t, t^{\prime} \in \tilde{\mathcal{T}}} \lambda\left(t, t^{\prime}\right)$. We say that a pair $t, t^{\prime}$ 
of terminals is $\mu$-element connected if and only if there are $\mu$ paths connecting $t$ to $t^{\prime}$ that are pairwise disjoint in both the edges and the nonterminal vertices of $\mathbf{G}$ (but they are allowed to share terminals). Let $\mu\left(t, t^{\prime}\right)$ be the largest value $\mu$ such that $t$ and $t^{\prime}$ are $\mu$-element connected and denote $\mu_{\mathbf{G}}(\tilde{\mathcal{T}})=\min _{t, t^{\prime} \in \tilde{\mathcal{T}}} \mu\left(t, t^{\prime}\right)$. Clearly, $\lambda_{\mathbf{G}}(\tilde{\mathcal{T}}) \geq \mu_{\mathbf{G}}(\tilde{\mathcal{T}})$ always holds. We use the following theorem several times.

Theorem 5.12. There is an efficient algorithm that, given a graph $\mathbf{G}$ and a set $\tilde{\mathcal{T}} \subseteq V(\mathbf{G})$ of $\kappa$ vertices called terminals, such that $\mu_{\mathbf{G}}(\tilde{\mathcal{T}}) \geq \mu$ for some $\mu \geq 1$, constructs another graph $\mathbf{H}$ with $V(\mathbf{H})=\tilde{\mathcal{T}}$, a partition $\mathcal{U}$ of $E(\mathbf{H})$ into groups of size at most $\kappa$, and for each edge $e=\left(t, t^{\prime}\right) \in E(\mathbf{H})$ a path $P_{e}$ connecting $t$ to $t^{\prime}$ in $\mathbf{G}$, such that

$-\lambda_{\mathbf{H}}(\tilde{\mathcal{T}}) \geq 2 \mu$

- for each terminal $t, d_{\mathbf{H}}(t) \leq 2 d_{\mathbf{G}}(t)$;

- for each e $\in E(\mathbf{H})$, path $P_{e}$ does not contain terminals as inner vertices;

- if $e^{\prime} \in E(\mathbf{G})$ is incident to a terminal of $\tilde{\mathcal{T}}$, then $e^{\prime}$ belongs to at most two paths in $\left\{P_{e} \mid e \in E(\mathbf{H})\right\} ;$ and

-if we select, for each group $U \in \mathcal{U}$, an arbitrary edge $e_{U} \in U$, then the corresponding paths $\left\{P_{e_{U}} \mid U \in \mathcal{U}\right\}$ are node disjoint in $\mathbf{G}$, except for possibly sharing endpoints.

Proof. We use the following theorem of Hind and Oellermann [1996] (see also Chekuri and Korula [2009]).

THEOREM 5.13. Let $\mathbf{G}$ be a graph, $\tilde{\mathcal{T}}$ a set of terminals in $\mathbf{G}$, and assume that $\mu_{\mathbf{G}}(\tilde{\mathcal{T}})=\mu$ for some $\mu \geq 0$. Let $(p, q)$ be an edge with $p, q \in V \backslash \tilde{\mathcal{T}}$. Let $G_{1}$ be the graph obtained from $G$ by deleting the edge $(p, q)$, and let $G_{2}$ be obtained from $G$ by contracting it. Then either $\mu_{G_{1}}(\tilde{\mathcal{T}}) \geq \mu$ or $\mu_{G_{2}}(\tilde{\mathcal{T}}) \geq \mu$.

While our graph $\mathbf{G}$ contains an edge $(p, q)$ connecting two nonterminal vertices $p$ and $q$, we apply Theorem 5.13 to $\mathbf{G}, \tilde{\mathcal{T}}$ and the edge $(p, q)$, and replace $\mathbf{G}$ with the resulting graph, where the edge $(p, q)$ is either deleted or contracted. Let $G^{\prime}$ be the graph obtained at the end of this procedure. For simplicity, we call the terminal vertices of $G^{\prime}$ black vertices and the nonterminal vertices white vertices. Let $W$ denote the set of all white vertices. Notice that every edge in $G^{\prime}$ either connects two black vertices or connects a white vertex to a black vertex. Moreover, we can assume without loss of generality that for each $t \in \tilde{\mathcal{T}}, v \in W$, there is at most one edge $(t, v)$ in $G^{\prime}$ : otherwise, if several such parallel edges are present, we delete all but one such edge. This does not affect the element connectivity of any pair $t, t^{\prime}$ of terminals, as the paths connecting them are not allowed to share $v$. Thus, we will assume from now on that every such pair $(t, v)$ at most one edge $(t, v)$ is present in $G^{\prime}$. Notice that for each terminal $t, d_{G^{\prime}}(t) \leq d_{G}(t)$. For every pair $\left(t, t^{\prime}\right)$ of terminals, an edge $\left(t, t^{\prime}\right)$ is present in $G^{\prime}$ if and only if it was present in G. Every white vertex $v$ is naturally associated with a connected subgraph $C_{v}$ of $\mathbf{G}$, containing all edges that were contracted into $v$, and all subgraphs $\left\{C_{v}\right\}_{v \in W}$ are completely disjoint. For each edge $(v, t)$ connecting $v$ to some terminal $t$ in $G^{\prime}$, there is an edge $(u, t)$ in $\mathbf{G}$, where $u$ is some vertex in $C_{v}$. Notice that $\lambda_{G^{\prime}}(\tilde{\mathcal{T}}) \geq \mu_{G^{\prime}}(\tilde{\mathcal{T}}) \geq \mu$.

Next, we replace every edge in $G^{\prime}$ by two parallel edges and denote the resulting graph by $G^{\prime \prime}$, so $G^{\prime \prime}$ is Eulerian. The degree of every terminal $t$ now becomes at most $2 d_{\mathbf{G}}(t)$, and $\lambda_{G^{\prime \prime}}(\tilde{\mathcal{T}}) \geq 2 \mu$. We now start constructing the final graph $\mathbf{H}$ and the partition $\mathcal{U}$ of its edges. We start with $\mathbf{H}=G^{\prime \prime}$, and for every edge $\left(t, t^{\prime}\right) \in E\left(G^{\prime}\right)$ connecting a pair $t, t^{\prime} \in \tilde{\mathcal{T}}$ of terminals, we add a new group $U$, containing the two copies of the edge $\left(t, t^{\prime}\right)$ in $G^{\prime \prime}$, to $\mathcal{U}$. Next, we take care of the white vertices by using the following edge-splitting operation due to Mader [1978].

TheOREM 5.14. Let $\mathbf{G}$ be an undirected multigraph, s a vertex of $\mathbf{G}$ whose degree is not 3 , such that $s$ is not incident to a cut edge of G. Then s has two neighbors $u$ and $v$, 
such that the graph $\mathbf{G}^{\prime}$, obtained from $\mathbf{G}$ by replacing the edges $(s, u)$ and $(s, v)$ with the edge $(u, v)$, satisfies $\lambda_{\mathbf{G}^{\prime}}(x, y)=\lambda_{\mathbf{G}}(x, y)$ for all $x, y \neq s$.

We process the white vertices $v \in W$ one by one. Consider some such vertex $v$. Recall that there are at most $2 \kappa$ edges incident to $v$ in $G^{\prime \prime}$. We apply Theorem 5.14 to vertex $v$ repeatedly until it becomes an isolated vertex (since the degree of $v$ is even due to the doubling of all edges, and the terminals are $2 \mu$-edge connected, the conditions of the theorem are always satisfied). Let $U_{v}$ be the set of all resulting new edges in graph $\mathbf{H}$. We add $U_{v}$ to $\mathcal{U}$. Notice that $\left|U_{v}\right| \leq \kappa$. Once all vertices $v \in W$ are processed, we obtain the final graph $\mathbf{H}$. It is easy to see that the degree of every terminal $t \in \tilde{\mathcal{T}}$ is at most $2 d_{\mathbf{G}}(t)$, as the edge-splitting operation does not change the degrees of the terminals. Every edge $e=\left(t, t^{\prime}\right)$ in $\mathbf{H}$ is naturally associated with a path $P_{e}$ connecting $t$ and $t^{\prime}$ in $\mathbf{G}$ : if edge $e=\left(t, t^{\prime}\right)$ is present in $\mathbf{G}$, then $P_{e}=e$. Otherwise, edge $e$ was obtained by replacing a pair $(t, v),\left(v, t^{\prime}\right)$ of vertices in $G^{\prime \prime}$ with edge $\left(t, t^{\prime}\right)$. In this case, there must be vertices $u, u^{\prime} \in C_{v}$ (possibly $u=u^{\prime}$ ), with edges $e_{1}=(u, t), e_{2}=\left(u^{\prime}, t^{\prime}\right) \in E(\mathbf{G})$. We let $P$ be a path connecting $u$ to $u^{\prime}$ in $C_{v}$ and set $P_{e}=\left(e_{1}, P, e_{2}\right)$. Given two edges $e, e^{\prime} \in E(\mathbf{H})$, the only possibility that the paths $P_{e}$ and $P_{e^{\prime}}$ share inner vertices or edges is when $e, e^{\prime}$ are two copies of the same edge connecting some pair of terminals in $G^{\prime \prime}$, or both edges belong to some set $U_{v}$, for some $v \in W$. Therefore, choosing at most one edge from each group $U \in \mathcal{U}$ ensures that the resulting paths are internally node- and edge disjoint.

Consider now some edge $e^{\prime} \in E(\mathbf{G})$ such that $e^{\prime}$ is incident to some terminal $t$. Notice that exactly two copies of $e^{\prime}$ were present in $\mathbf{H}$ before the edge-splitting procedure. It is then immediate to verify that $e^{\prime}$ may belong to at most two paths in set $\left\{\mathcal{P}_{e} \mid e \in\right.$ $E(\mathbf{H})\}$.

5.4.1. Phase 1. Let $H$ be the graph obtained from $G$ by contracting each cluster $S_{i} \in \mathcal{R}$ into a supernode $v_{i}$. Let $\tilde{\mathcal{T}}=\left\{v_{1}, \ldots, v_{\ell_{0}}\right\}$ be the resulting set of supernodes that we will refer to as terminals in this phase. As observed previously, every pair $S_{i}, S_{j} \in \mathcal{R}^{\prime}$ of clusters has at least $\mu=\left\lfloor\frac{w_{0}}{6 \Delta}\right\rfloor$ node-disjoint paths connecting them in $G$. Therefore, $\mu_{H}(\tilde{\mathcal{T}}) \geq \mu$. We apply Theorem 5.12 to graph $H$, set $\tilde{\mathcal{T}}$ of terminals, and the value $\mu$. Let $H^{\prime}$ denote the resulting graph and $\mathcal{U}$ the resulting partition of the edges of $H^{\prime}$, where each group $U \in \mathcal{U}$ contains at most $\ell_{0}$ edges. Recall that each edge $e=\left(v_{i}, v_{j}\right)$ in $H^{\prime}$ corresponds to a path $P_{e}$ connecting $v_{i}$ to $v_{j}$ in $H$, where $P_{e}$ does not contain vertices of $\tilde{\mathcal{T}}$, except for its endpoints. In turn, path $P_{e}$ defines a path $P_{e}^{\prime}$ in graph $G$, connecting a vertex of $S_{j}$ to a vertex of $S_{i}$ directly-that is, $P_{e}^{\prime}$ does not contain the vertices of $\bigcup_{S \in \mathcal{R}} S$ as inner vertices.

Let $w_{1}=\left\lfloor\frac{w_{0}}{2 \Delta \ell_{0}^{2}}\right\rfloor$. We define a new graph $Z$, whose vertex set is $\left\{v_{1}, \ldots, v_{\ell_{0}}\right\}$. We add an edge $\left(v_{i}, v_{j}\right)$ to $Z$ if and only if there are at least $w_{1}$ parallel edges connecting $v_{i}$ to $v_{j}$ in $H^{\prime}$. It is easy to verify that graph $Z$ is connected: indeed, assume otherwise. Let $A$ be some connected component of $Z$, and let $B$ contain the rest of the vertices. Let $v_{j} \in A$, $v_{j^{\prime}} \in B$. Since there are at least $2 \mu \geq\left\lfloor\frac{w_{0}}{6 \Delta}\right\rfloor$ edge-disjoint paths connecting $v_{j}$ and $v_{j^{\prime}}$ in $H^{\prime},\left|E_{H^{\prime}}(A, B)\right| \geq\left\lfloor\frac{w_{0}}{6 \Delta}\right\rfloor$ must hold. Since $|A|+|B|=\ell_{0}$, at least one pair $\left(v_{j}, v_{j^{\prime}}\right)$ with $v_{j} \in A, v_{j^{\prime}} \in B$, has at least $w_{1}$ parallel edges connecting them in $H^{\prime}$.

Let $T$ be a spanning tree of $Z$ that is rooted at some arbitrary node. We say that Case 1 happens if $T$ contains a root-to-leaf path of length at least $\ell$, and we say that Case 2 happens otherwise. Since $|V(T)|=\ell_{0}=\ell^{2}$, and every vertex of $T$ lies on some root-to-leaf path, if Case 2 happens, then $T$ contains at least $\ell$ leaves (see Claim 2.2). We now consider each of the two cases separately. For Case 1, we build a good tree-of-sets system directly, and we only apply Phase 2 of the algorithm if Case 2 happens. 
Case 1. Let $P$ be a path of $T$ of length exactly $\ell$. Assume without loss of generality that $P=\left\{v_{1}, \ldots, v_{\ell}\right\}$. Let $\mathcal{R}^{\prime}=\left\{S_{1}, \ldots, S_{\ell}\right\}$ be the set of corresponding clusters. We build a tree-of-sets system $\left(\mathcal{R}^{\prime}, T^{*}, \bigcup_{e \in T^{*}} \mathcal{P}_{e}^{*}\right)$. The tree $T^{*}$ is just a path connecting $v_{1}, \ldots, v_{\ell}$ in this order. To define the sets $\mathcal{P}_{e}^{*}$ of edges, we perform the following procedure. Recall that each edge $\left(v_{i}, v_{i+1}\right)$ in $P$ corresponds to a collection $E_{i}$ of at least $w_{1}$ edges in graph $H^{\prime}$. For each group $U \in \mathcal{U}$, we select one edge $e_{U} \in U$ uniformly at random. Let $E^{*}=\left\{e_{U} \mid U \in \mathcal{U}\right\}$ be the set of the selected edges. For each $1 \leq i<\ell$, let $E_{i}^{\prime}=E_{i} \cap E^{*}$. Since the size of each group $U$ is at most $\ell_{0}$, the expected number of edges in $E_{i}^{\prime}$ is at least $\frac{w_{1}}{\ell_{0}}>w \geq \log k$. Using the standard Chernoff bound, with high probability, for each $1 \leq i<\ell,\left|E_{i}^{\prime}\right| \geq\left\lfloor\frac{w_{1}}{2 \ell_{0}}\right\rfloor$. Let $w_{2}=\left\lfloor\frac{w_{1}}{2 \ell_{0}}\right\rfloor$. If $E_{i}^{\prime}$ contains more than $w_{2}$ edges, we discard arbitrary edges from $E_{i}^{\prime}$ until $\left|E_{i}^{\prime}\right|=w_{2}$ holds. Let $\tilde{\mathcal{P}}_{i}=\left\{P_{e} \mid e \in E_{i}^{\prime}\right\}$, and let $\tilde{\mathcal{P}}=\bigcup_{i=1}^{\ell-1} \tilde{\mathcal{P}}_{i}$. Then from Theorem 5.12 , the paths in $\tilde{\mathcal{P}}$ are internally node disjoint in $H$, and they do not contain terminals as inner vertices. Therefore, the corresponding paths in graph $G$ are also internally node disjoint, and they do not contain the vertices of $\bigcup_{i=1}^{\ell} S_{i}$ as inner vertices. For each $1 \leq i \leq \ell$, let $\mathcal{P}_{i}$ be the set of paths in graph $G$, corresponding to the paths in $\tilde{\mathcal{P}}_{i}$, and let $\mathcal{P}=\bigcup_{i=1}^{\ell-1} \mathcal{P}_{i}$. Then the paths in $\mathcal{P}$ are direct and internally node disjoint. Only two issues remain. First, it is possible that some paths in $\mathcal{P}$ share endpoints, and second, we need to ensure that the clusters $S_{i}$ have the bandwidth property in the corresponding graph.

To solve the first problem, we will define, for each $1 \leq i<\ell$, a subset $\mathcal{P}_{i}^{\prime} \subseteq \mathcal{P}_{i}$ of $w$ paths such that every vertex of $\bigcup_{i=1}^{\ell} S_{i}$ belongs to at most one path in $\bigcup_{i^{\prime}=1}^{\ell-1} \mathcal{P}_{i^{\prime}}^{\prime}$ (or in other words, the paths in $\bigcup_{i^{\prime}=1}^{\ell-1} \mathcal{P}_{i^{\prime}}^{\prime}$ have distinct endpoints).

We start with the set $\mathcal{P}_{1}$. Using Claim 2.3 , we can compute a subset $\mathcal{P}_{1}^{\prime} \subseteq \mathcal{P}_{1}$ of $\left\lfloor\frac{w_{2}}{2 \Lambda}\right\rfloor$ paths that do not share endpoints in $S_{1}$ or $S_{2}$. We then consider the set $\mathcal{P}_{2}$ of paths. We delete from $\mathcal{P}_{2}$ all paths that share endpoints with paths in $\mathcal{P}_{1}^{\prime}$. Since $\left|\mathcal{P}_{1}^{\prime}\right|=\left\lfloor\frac{w_{2}}{2 \Delta}\right\rfloor$, at most $\frac{w_{2}}{2}$ paths of $\mathcal{P}_{2}$ may share endpoints with paths in $\mathcal{P}_{1}^{\prime}$, so at least half the paths in $\mathcal{P}_{2}$ remain. As before, we select a subset $\mathcal{P}_{2}^{\prime}$ of $\left\lfloor\frac{w_{2}}{2 \Delta}\right\rfloor$ such paths that do not endpoints using Claim 2.3. We continue this process for all $1 \leq i<\ell$ until all paths in set $\mathcal{P}^{\prime}=\bigcup_{i} \mathcal{P}_{i}^{\prime}$ are mutually disjoint. The sets $\mathcal{P}_{i}^{\prime}$ of paths are then used to define the sets $\mathcal{P}_{e}^{*}$ of paths in the tree-of-sets system. Notice that the size of each set is $\left\lfloor\frac{w_{2}}{2 \Delta^{2}}\right\rfloor \geq \frac{w_{1}}{8 \ell_{0} \Delta^{2}} \geq \frac{w_{0}}{32 \Delta^{3} \ell_{0}^{3}}>w$ from Equation (1).

Let $G^{*}$ be the subgraph of $G$ obtained by the union of $G[S]$ for $S \in \mathcal{R}^{\prime}$ and $\bigcup_{e \in T^{*}} \mathcal{P}_{e}^{*}$. We need to verify that each set $S_{i}$ has the $\alpha_{\mathrm{BW}}$-bandwidth property in $G^{*}$. Let $\Gamma_{i}$ be the interface of the set $S_{i}$ in $G^{*}$. We set up an instance of the sparsest cut problem with the graph $G\left[S_{i}\right]$ and the set $\Gamma_{i}$ of terminals, and apply algorithm $\mathcal{A}_{\mathrm{ARV}}$ to it. If the outcome is a cut of sparsity less than $\alpha$, then since $\left|\Gamma_{i}\right|<w_{0}$, we obtain an $\left(w_{0}, \alpha\right)$-violating cut of $S_{i}$ in graph $G$. We return this cut as the outcome of the algorithm. If $\mathcal{A}_{\mathrm{ARV}}$ returns a cut of sparsity at least $\alpha$ for each set $S_{i}$, for $1 \leq i \leq \ell$, we are guaranteed that each such set has the $\alpha_{\mathrm{BW}}$-bandwidth property in $G^{*}$, and we have therefore constructed a good tree-of-sets system. (We are guaranteed that $S_{i} \cap \mathcal{T}=\emptyset$ for each $i$, as each set $S_{i} \in \mathcal{R}$ only contains nonterminal vertices.)

Case 2. If Case 2 happens, then we need to execute the second phase of the algorithm, but first we need to establish the following useful fact. Recall that we have found a tree $T$ in graph $Z$ containing at least $\ell$ leaves. Let $\mathcal{R}^{\prime} \subseteq \mathcal{R}$ be an arbitrary subset of $\ell$ clusters corresponding to the leaves of $T$. For simplicity of notation, we assume that $\mathcal{R}^{\prime}=\left\{S_{1}, \ldots, S_{\ell}\right\}$. We say that a path $P$ connects $S_{i}$ to $S_{j}$ directly for $S_{i}, S_{j} \in \mathcal{R}^{\prime}$ if and only if no inner vertex of $P$ belongs to $\bigcup_{S \in \mathcal{R}^{\prime}} S$ (but they may belong to clusters $\left.S \in \mathcal{R} \backslash \mathcal{R}^{\prime}\right)$. 
THEOREM 5.15. There is an efficient randomized algorithm that with high probability either computes a $\left(w_{0}, \alpha\right)$-violating cut of some set $S \in \mathcal{R}$ or finds, for each set $S_{i} \in \mathcal{R}^{\prime}$, a subset $E_{i} \subseteq \operatorname{out}_{G}\left(S_{i}\right)$ of edges, and for each $1 \leq i \neq j \leq \ell$ a collection $\mathcal{P}_{i, j}$ of paths in $G$ that satisfy the following properties:

$-\left|E_{i}\right|=w_{3}=\Omega\left(\frac{\alpha^{2} w_{1}}{\ell \Delta^{7} \log k}\right):$

- $\mathcal{P}_{i, j}$ is a collection of $w_{3}$ node-disjoint paths that directly connect $S_{i}$ to $S_{j}$; and

- each path $P \in \mathcal{P}_{i, j}$ contains an edge of $E_{i}$ and an edge of $E_{j}$ as its first and last edges, respectively.

Notice that the theorem implies that the edges in each set $E_{i}$ do not share endpoints.

Proof. Recall that each edge $e \in E\left(H^{\prime}\right)$ corresponds to a path $P_{e}$ in $H$. Since for any choice of edges $e_{U} \in U$ for each $U \in \mathcal{U}$, we are guaranteed that the corresponding set $\left\{P_{e_{U}} \mid U \in \mathcal{U}\right\}$ of paths is edge disjoint in $H$, and $|U| \leq \ell_{0}$ for all $U \in \mathcal{U}$, the total edge congestion in $H$ due to the paths in $\left\{P_{e} \mid e \in E\left(H^{\prime}\right)\right\}$ is at most $\ell_{0}$. Each such path $P_{e}$ naturally defines a direct path $P_{e}^{\prime}$ in $G$, and the total edge congestion due to the paths in $\left\{P_{e}^{\prime} \mid e \in E\left(H^{\prime}\right)\right\}$ is at most $\ell_{0}$ in $G$.

Let $v^{*}$ be the root of the tree $T$, and let $S^{*} \in \mathcal{R}$ be the corresponding vertex subset. We use the following claim.

CLAIM 5.16. For each set $S_{j} \in \mathcal{R}^{\prime}$, there is a flow $F_{j}$ of value $\left\lceil\frac{w_{1}}{2 \Delta}\right\rceil$, originating from the vertices of $S^{*}$ and terminating at the vertices of $S_{j}$ in $G$, with edge-congestion at most $\frac{2}{\alpha}$, such that the flow-paths in $F_{j}$ do not contain the vertices of $\bigcup_{S \in \mathcal{R}^{\prime}} S$ as inner vertices.

Proof. Consider the path $\left(v^{*}=v_{i_{1}}, v_{i_{2}}, \ldots, v_{i_{x}}=v_{j}\right)$ in the tree $T$, connecting $v^{*}$ to $v_{j}$. For each edge $e_{z}=\left(v_{i_{z}}, v_{i_{z+1}}\right)$ on this path, there are $w_{1}$ parallel edges corresponding to $e_{z}$ in graph $H^{\prime}$. Let $\mathcal{Q}_{z}$ be the corresponding set of $w_{1}$ direct paths connecting the vertices of $S_{i_{z}}$ to the vertices of $S_{i_{z+1}}$ in $G$. Recall that from Theorem 5.12, every edge incident to a vertex of $S_{i_{z}} \cup S_{i_{z+1}}$ belongs to at most two paths in set $\mathcal{Q}_{z}$. Therefore, every vertex of $S_{i_{z}} \cup S_{i_{z+1}}$ serves as an endpoint of at most $2 \Delta$ paths. We can construct a bipartite graph, whose vertex set is $\left(S_{i_{z}} \cup S_{i_{z+1}}\right)$, and there is an edge $(u, v)$ for $u \in S_{i_{z}}$, $v \in S_{i_{z+1}}$ for every path in $\mathcal{Q}_{z}$ connecting $u$ to $v$. Then the resulting bipartite graph has maximum vertex degree at most $2 \Delta$, and thus from Claim 2.3, we can find a matching of cardinality $\left\lceil\frac{\left|\mathcal{Q}_{z}\right|}{2 \Delta}\right\rceil$ in this graph. This matching defines a subset $\mathcal{Q}_{z}^{\prime} \subseteq \mathcal{Q}_{z}$ of $\left\lceil\frac{w_{1}}{2 \Delta}\right\rceil$ paths, whose endpoints are all distinct. Denote

$$
\Gamma_{z}^{2}=\left\{v \in S_{i_{z}} \mid v \text { is the first vertex on some path in } \mathcal{Q}_{z}^{\prime}\right\},
$$

and similarly,

$$
\Gamma_{z+1}^{1}=\left\{v \in S_{i_{z+1}} \mid v \text { is the last vertex on some path in } \mathcal{Q}_{z}^{\prime}\right\} .
$$

For each $1<z<x$, we have now defined two subsets $\Gamma_{z}^{1}, \Gamma_{z}^{2} \subseteq S_{i_{z}}$ of vertices of cardinality $\left\lceil\frac{w_{1}}{2 \Delta}\right\rceil<w_{0}$ each. Let $n_{z}=\left|\Gamma_{z}^{1} \backslash \Gamma_{z}^{2}\right|=\left|\Gamma_{z}^{2} \backslash \Gamma_{z}^{1}\right|$. We now try to send flow in $G\left[S_{i_{z}}\right]$, where every vertex in $\Gamma_{z}^{1} \backslash \Gamma_{z}^{2}$ sends one flow unit, and every vertex in $\Gamma_{z}^{2} \backslash \Gamma_{z}^{1}$ receives one flow unit, with edge congestion at most $1 / \alpha$. If such a flow does not exist, then the minimum edge cut separating these two vertex subsets defines a $\left(w_{0}, \alpha\right)$ violating cut of $S_{i_{z}}$. We then terminate the algorithm and return this cut. We assume therefore that such a flow exists and denote it by $F_{z}^{\prime}$.

Concatenating the flows $\left(\mathcal{Q}_{1}^{\prime}, F_{1}^{\prime}, \mathcal{Q}_{2}^{\prime}, F_{2}^{\prime}, \ldots, F_{x-1}^{\prime}, \mathcal{Q}_{x-1}^{\prime}\right)$, we obtain the desired flow $F_{j}$ of value $\left\lceil\frac{w_{1}}{2 \Delta}\right\rceil$. The total congestion caused by paths in $\bigcup_{z=1}^{x-1} \mathcal{Q}_{z}$ is at most $\ell_{0}$, whereas each flow $F_{z}^{\prime}$ causes congestion at most $1 / \alpha$ in graph $G\left[S_{i_{z}}\right]$. Therefore, the total congestion due to flow $F_{j}$ is bounded by $\frac{1}{\alpha}+\ell_{0} \leq \frac{2}{\alpha}$. 
Scaling all flows $F_{j}$, for $S_{j} \in \mathcal{R}^{\prime}$ down by factor $2 \ell \Delta / \alpha$, we obtain a new flow $F$, where every set $S_{j} \in \mathcal{R}^{\prime}$ sends at least $\left\lfloor\frac{w_{1} \alpha}{4 \ell \Delta^{2}}\right\rfloor$ flow units to $S^{*}$, and the total vertex congestion due to $F$ is at most 1 . The flow-paths of $F$ do not contain the vertices of $\bigcup_{j=1}^{\ell} S_{j}$ as inner vertices. We now use the following claim.

CLAIM 5.17. There is an efficient algorithm to compute a collection $\left\{\mathcal{P}_{j}\right\}_{j=1}^{\ell}$ of path sets, where for each $1 \leq j \leq \ell$, set $\mathcal{P}_{j}$ contains $\left\lfloor\frac{w_{1} \alpha}{4 \ell \Delta^{2}}\right\rfloor$ paths connecting $S_{j}$ to $S^{*}$, the paths in $\bigcup_{j=1}^{\ell} \mathcal{P}_{j}$ are node disjoint, and they do not contain the vertices of $\bigcup_{j=1}^{\ell} S_{j}$ as inner vertices.

Proof. We set up a flow network $\mathcal{N}$ : start with the graph $G$ and bi-direct all of its edges, setting the capacity of every vertex to 1 . Delete all edges entering the clusters $S_{j}$, and all edges with both endpoints in $S_{j}$, for all $1 \leq j \leq \ell$. Delete all edges leaving the cluster $S^{*}$ and every edge with both endpoints in $S^{*}$. For each $1 \leq j \leq \ell$, add a vertex $s_{j}$ of capacity $\left\lfloor\frac{w_{1} \alpha}{4 \ell \Delta^{2}}\right\rfloor$, and connect $s_{j}$ to every vertex of $S_{j}$ with a directed edge. Finally, add a source vertex $s$ of infinite capacity, connecting it to every vertex in $\left\{s_{1}, \ldots, s_{\ell}\right\}$, and add a destination vertex $t$ of infinite capacity, to which every vertex of $S^{*}$ connects. Notice that flow $F$ defines a feasible $s$ - $t$ flow of value $\ell \cdot\left\lfloor\frac{w_{1} \alpha}{4 \ell \Delta^{2}}\right\rfloor$ in this network. From the integrality of flow, there is an integral flow of this value in $\mathcal{N}$. It is immediate to verify that this integral flow defines the desired collections $\mathcal{P}_{j}$ of paths for $1 \leq j \leq \ell$.

For each $1 \leq j \leq \ell$, let $A_{j} \subseteq S^{*}$ be the set of vertices that serve as endpoints of paths in $\mathcal{P}_{j}$, and let $A=\bigcup_{j=1}^{\ell} A_{j}$. Notice that $|A|=\ell \cdot\left\lceil\frac{w_{1} \alpha}{4 \ell \Delta^{2}}\right\rceil<w_{0}$. We set up an instance of the sparsest cut problem in graph $G\left[S^{*}\right]$, where the vertices of $A$ act as terminals, and apply algorithm $\mathcal{A}_{\mathrm{ARV}}$ to this problem. If the algorithm returns a cut whose sparsity is less than $\alpha$, then we have found a $\left(w_{0}, \alpha\right)$-violating cut in $S^{*} \in \mathcal{R}$. We return this cut and terminate the algorithm. Otherwise, we are guaranteed that the set $A$ is $\alpha_{\mathrm{BW}}$-well-linked in $G\left[S^{*}\right]$.

We apply Corollary 2.15 to graph $G\left[S^{*}\right]$ and the sets $A_{1}, \ldots, A_{\ell}$ of vertices, obtaining, for each $1 \leq j \leq \ell$, a subset $A_{j}^{*} \subseteq A_{j}$ of $\Omega\left(\frac{\alpha_{\mathrm{BW}}\left|A_{j}\right|}{\Delta^{5} \sqrt{\log k}}\right)=\Omega\left(\frac{\alpha^{2} w_{1}}{\ell \Delta^{7} \log k}\right)=w_{3}$ vertices, such that for all $1 \leq i \neq j \leq \ell, A_{j}^{*}$ and $A_{i}^{*}$ are linked in $G\left[S^{*}\right]$. Let $\mathcal{Q}_{i, j}$ be the set of $w_{3}$ node-disjoint paths connecting $A_{j}^{*}$ to $A_{i}^{*}$ in $G\left[S^{*}\right]$.

For each $1 \leq j \leq \ell$, let $\mathcal{P}_{j}^{\prime} \subseteq \mathcal{P}_{j}$ be the subset of paths whose endpoint belongs to $A_{j}^{*}$, and let $E_{j} \subseteq \operatorname{out}\left(S_{j}\right)$ be the set of edges $e$, where $e$ is the first edge on some path of $\mathcal{P}_{j}^{\prime}$, so $\left|E_{j}\right|=w_{3}$. Consider a pair $1 \leq i<j \leq \ell$ of indices. The desired set of paths connecting $S_{i}$ to $S_{j}$ is obtained by concatenating the paths in $\mathcal{P}_{i}^{\prime}, \mathcal{Q}_{i, j}$, and $\mathcal{P}_{j}^{\prime}$.

To summarize, we have found a collection $\mathcal{R}^{\prime}=\left\{S_{1}, \ldots, S_{\ell}\right\}$ of $\ell$ disjoint vertex subsets, and for each set $S_{j}$, a collection $E_{j} \subseteq$ out $_{G}\left(S_{j}\right)$ of $w_{3}$ edges, such that for each $1 \leq j \neq i \leq \ell$, there is a set of $w_{3}$ node-disjoint paths in $G$, connecting $S_{i}$ to $S_{j}$ directly, such that each path contains an edge of $E_{j}$ and an edge of $E_{i}$ as its first and last edges, respectively.

5.4.2. Phase 2. We construct a new graph $\tilde{H}$, obtained from $G$ as follows. First, for each $1 \leq j \leq \ell$, we delete all edges in out $\left(S_{j}\right) \backslash E_{j}$ from $G$. Let $B_{j} \subseteq S_{j}$ be the subset of vertices containing the endpoints of the edges in $E_{j}$ that belong to $S_{j}$. We delete all vertices of $S_{j} \backslash B_{j}$ and add a new supernode $v_{j}$ that connects to every vertex in $B_{j}$ with an edge. Recall that from the preceding discussion, $\left|B_{j}\right|=w_{3}$, so the degree of every supernode $v_{j}$ in $\tilde{H}$ is $w_{3}$, and every pair $v_{j}, v_{i}$ of supernodes are connected by $w_{3}$ paths, which are completely disjoint, except for sharing the first and the last vertex. We will think of the supernode $v_{j}$ as representing the set $S_{j} \in \mathcal{R}^{\prime}$. In this phase, the vertices of 
$\left\{v_{1}, \ldots, v_{\ell}\right\}$ are called terminals, and a path $P$ connecting a vertex of $S_{i}$ to a vertex of $S_{i^{\prime}}$ in $G$ is called direct if and only if it does not contain the vertices of $\bigcup_{j=1}^{\ell} S_{j}$ as inner vertices.

We apply Theorem 5.12 to graph $\tilde{H}$ with the set $\left\{v_{1}, \ldots, v_{\ell}\right\}$ of terminals and $\mu=w_{3}$. Let $\tilde{H}^{\prime}$ denote the resulting graph, and let $\mathcal{U}$ denote the resulting partition of the edges of $\tilde{H}$ into groups of size at most $\ell$. Recall that the degree of every vertex of $\tilde{H}^{\prime}$ is at most $2 w_{3}$, and every pair of vertices is $\left(2 w_{3}\right)$-edge connected. This can only happen if the degree of every vertex is exactly $2 w_{3}$. The main difference between graph $\tilde{H}^{\prime}$ and the graph $H^{\prime}$ that we computed in Phase 1 is that now the degree of every terminal and the edge connectivity of every pair of terminals are the same. It is this property that allows us to build the tree-of-sets system. To simplify notation, denote $h=2 w_{3}$.

Suppose that we choose, for each group $U \in \mathcal{U}$, some edge $e_{U} \in U$. Then Theorem 5.12 guarantees that all paths in $\left\{P_{e_{U}} \mid U \in \mathcal{U}\right\}$ are node disjoint in $\tilde{H}$, except for possibly sharing endpoints, and they do not contain terminals as inner vertices. For each such edge $e_{U}=\left(v_{i}, v_{j}\right) \in E\left(\tilde{H}^{\prime}\right)$, path $P_{e_{U}}$ in $\tilde{H}$ naturally defines a direct path $P_{e_{U}}^{\prime}$, connecting a vertex of $S_{i}$ to a vertex of $S_{j}$ in $G$. Moreover, from the definition of graph $\tilde{H}$, the paths in $\left\{P_{e_{U}}^{\prime} \mid U \in \mathcal{U}\right\}$ are completely node disjoint in $G$. For each edge $e \in E\left(\tilde{H}^{\prime}\right)$, let $P_{e}^{\prime}$ denote the path in graph $G$ corresponding to the path $P_{e}$ in $\tilde{H}$.

We build an auxiliary undirected graph $\tilde{Z}$ on the set $\left\{v_{1}, \ldots, v_{\ell}\right\}$ of vertices as follows. For each pair $v_{j}, v_{j^{\prime}}$ of vertices, there is an edge $\left(v_{j}, v_{j^{\prime}}\right)$ in graph $\tilde{Z}$ if and only if there are at least $h / \ell^{3}$ edges connecting $v_{j}$ and $v_{j^{\prime}}$ in $\tilde{H}^{\prime}$. If edge $e=\left(v_{j}, v_{j^{\prime}}\right)$ is present in graph $\tilde{Z}$, then its capacity $c(e)$ is set to be the number of edges connecting $v_{j}$ to $v_{j^{\prime}}$ in $\tilde{H}^{\prime}$. For each vertex $v_{j}$, let $C\left(v_{j}\right)$ denote the total capacity of edges incident on $v_{j}$ in graph $\tilde{Z}$. We need the following simple observation.

OBSERVATION 5.18.

-For each vertex $v \in V(\tilde{Z}),\left(1-1 / \ell^{2}\right) h \leq C(v) \leq h$.

-For each pair $(u, v)$ of vertices in graph $\tilde{Z}$, we can send at least $(1-1 / \ell) h$ flow units from $u$ to $v$ in $\tilde{Z}$ without violating the edge capacities.

Proof. To prove the first assertion, recall that each vertex in graph $\tilde{H}^{\prime}$ has $h$ edges incident to it. Thus, $C(v) \leq h$ for all $v \in V(\tilde{Z})$. Call a pair $\left(v_{j}, v_{j^{\prime}}\right)$ of vertices bad if and only if there are fewer than $h / \ell^{3}$ edges connecting $v_{j}$ to $v_{j^{\prime}}$ in $\tilde{H}^{\prime}$. Notice that each vertex $v \in V(\tilde{Z})$ may participate in at most $\ell$ bad pairs, as $|V(\tilde{Z})|=\ell$. Therefore, $C(v) \geq h-\ell h / \ell^{3}=h\left(1-1 / \ell^{2}\right)$ must hold.

For the second assertion, assume for contradiction that it is not true, and let $(u, v)$ be a violating pair of vertices. Then there is a cut $(A, B)$ in $\tilde{Z}$, with $u \in A, v \in B$, and the total capacity of edges crossing this cut is at most $(1-1 / \ell) h$. Since $u$ and $v$ were connected by $h$ edge-disjoint paths in graph $\tilde{H}^{\prime}$, this means that there are at least $h / \ell$ edges in graph $\tilde{H}^{\prime}$ that connect bad pairs of vertices. But since we can only have at most $\ell^{2}$ bad pairs, and each pair has fewer than $h / \ell^{3}$ edges connecting them, this is impossible.

The following claim allows us to find a spanning tree of $\tilde{Z}$ with maximum vertex degree at most 3 . It relies on the specific properties of the graph $\tilde{Z}$ outlined in the preceding observation. This low-degree spanning tree will be used to define the treeof-sets system.

ClaIM 5.19. There is an efficient algorithm to find a spanning tree $T^{*}$ of $\tilde{Z}$ with maximum vertex degree at most 3. 
Proof. We use the algorithm of Singh and Lau [2015] for constructing boundeddegree spanning trees. Suppose that we are given a graph $G=(V, E)$, and our goal is to construct a spanning tree $T$ of $G$, where the degree of every vertex is bounded by some integer $B$. For each subset $S \subseteq V$ of vertices, let $E(S)$ denote the subset of edges with both endpoints in $S$, and let $\delta(S)$ denote the subset of edges with exactly one endpoint in $S$. Singh and Lau consider a natural LP-relaxation for the problem. We note that their algorithm works for a more general problem where edges are associated with costs, and the goal is to find a minimum-cost tree that respects the degree requirements; since we do not need to minimize the tree cost, we only discuss the unweighted version here. For each edge $e \in E$, we have a variable $x_{e}$ indicating whether $e$ is included in the solution. We are looking for a feasible solution to the following LP:

$$
\begin{gathered}
\sum_{e \in E} x_{e}=|V|-1, \\
\sum_{e \in E(S)} x_{e} \leq|S|-1 \quad \forall S \subsetneq V, \\
\sum_{e \in \delta(v)} x_{e} \leq B \quad \forall v \in V, \\
x_{e} \geq 0 \quad \forall e \in E .
\end{gathered}
$$

Singh and Lau [2015] show an efficient algorithm that, given a feasible solution to the preceding LP, produces a spanning tree $T$, where for each vertex $v \in V$, the degree of $v$ is at most $B+1$ in $T$. Therefore, to prove the claim, it suffices to show a feasible solution to the LP, where $B=2$. Recall that $|V(\tilde{Z})|=\ell$. The solution is defined as follows. Let $e=(u, v)$ be any edge in $E(\tilde{Z})$. We set the LP value of $e$ to be $x_{e}=\frac{\ell-1}{\ell} \cdot\left(\frac{c(e)}{C(v)}+\frac{c(e)}{C(u)}\right)$. We say that $\frac{\ell-1}{\ell} \cdot \frac{c(e)}{C(v)}$ is the contribution of $v$ to $x_{e}$ and $\frac{\ell-1}{\ell} \cdot \frac{c(e)}{C(u)}$ is the contribution of $u$. We now verify that all constraints of the LP hold.

First, it is easy to see that $\sum_{e \in E} x_{e}=\ell-1$, as required. Next, consider some subset $S \subsetneq V$ of vertices. Notice that it suffices to establish Constraint (3) for subsets $S$ with $|S| \geq 2$. From Observation 5.18, the total capacity of edges in $E_{\tilde{Z}}(S, \bar{S})$ must be at least $(1-1 / \ell) h$. Since for each $v \in S, C(v) \leq h$, the total contribution of the vertices in $S$ toward the LP weights of edges in $E_{\tilde{Z}}(S, \bar{S})$ is at least $\frac{\ell-1}{\ell} \cdot(1-1 / \ell)=(1-1 / \ell)^{2}$. Therefore,

$$
\sum_{e \in E(S)} x_{e} \leq \frac{\ell-1}{\ell}|S|-(1-1 / \ell)^{2}=|S|-|S| / \ell-1-1 / \ell^{2}+2 / \ell \leq|S|-1,
$$

as we assume that $|S| \geq 2$. This establishes Constraint (3). Finally, we show that for each $v \in V(\tilde{Z}), \sum_{e \in \delta_{v}} x_{e} \leq 2$. First, the contribution of the vertex $v$ to this summation is bounded by 1 . Next, recall that for each $u \in V(\tilde{Z}), C(u) \geq\left(1-1 / \ell^{2}\right) h$, whereas the total capacity of edges in $\delta(v)$ is at most $h$. Therefore, the total contribution of other vertices to this summation is bounded by $\frac{h}{\left(1-1 / \ell^{2}\right) h} \cdot \frac{\ell-1}{\ell} \leq \frac{\ell}{\ell+1} \leq 1$. The algorithm of Singh and Lau can now be used to obtain a spanning tree $T^{*}$ for $\tilde{Z}$ with maximum vertex degree at most 3.

We are now ready to define the tree-of-sets system $\left(\mathcal{R}^{\prime}, T^{*}, \bigcup_{e \in E\left(T^{*}\right)} \mathcal{P}^{*}(e)\right)$. The tree $T^{*}$ is the tree computed by Claim 5.19. To define the sets $\mathcal{P}^{*}(e)$ of paths, recall that each 
edge $e$ of $\tilde{Z}$ (and hence of $T^{*}$ ) corresponds to a set $S_{e}$ of at least $2 w_{3} / \ell^{3}$ edges of $\tilde{H}^{\prime}$. For each group $U \in \mathcal{U}$, we randomly choose one edge $e_{U} \in \mathcal{U}$, and we let $E^{*} \subseteq E(\tilde{H})$ be the set of all selected edges. For each edge $e \in E\left(T^{*}\right)$, let $S_{e}^{\prime}=S_{e} \cap E^{*}$. The expected size of $S_{e}^{\prime}$ is at least $\frac{2 w_{3}}{\ell^{4}}$, and using the standard Chernoff bound, with high probability, for each edge $e \in E\left(T^{*}\right),\left|S_{e}^{\prime}\right| \geq \frac{w_{3}}{\ell^{4}}$, since $w_{3} / \ell^{4} \geq w \geq 4 \log k$. This is because $\frac{w_{3}}{\ell^{4}}=\Omega\left(\frac{w_{1} \alpha^{2}}{\ell^{5} \Delta^{7} \log k}\right)=$ $\Omega\left(\frac{w_{0} \alpha^{2}}{\Delta^{8} \ell^{9} \log k}\right) \geq w$ from Equation (1). The final set $\mathcal{P}_{e}^{*}$ of paths is $\left\{P_{e^{\prime}}^{\prime} \mid e^{\prime} \in S_{e}^{\prime}\right\}$. Notice that $\left|\mathcal{P}_{e}^{*}\right| \geq w_{3} / \ell^{4} \geq w$. We delete paths from $\mathcal{P}_{e}^{*}$ as necessary until $\left|\mathcal{P}_{e}^{*}\right|=w$. From the definition of the graph $\tilde{H}$, and from Theorem 5.12, all paths in $\bigcup_{e \in E\left(T^{*}\right)} \mathcal{P}_{e}^{*}$ are mutually node disjoint.

Let $G^{*}$ be the subgraph of $G$ obtained by taking the union of $G\left[S_{j}\right]$ for $S_{j} \in \mathcal{R}^{\prime}$ and $\bigcup_{e \in E\left(T^{*}\right)} \mathcal{P}_{e}^{*}$. We need to verify that each set $S_{i}$ has the $\alpha_{\mathrm{BW}}$-bandwidth property in $G^{*}$. Let $\Gamma_{i}$ be the interface of the set $S_{i}$ in $G^{*}$. We set up a sparsest cut problem instance with the graph $G\left[S_{i}\right]$ and the set $\Gamma_{i}$ of terminals, and apply algorithm $\mathcal{A}_{\mathrm{ARV}}$ to it. If the outcome is a cut of sparsity less than $\alpha$, then, since $\left|\Gamma_{i}\right|<w_{0}$, we obtain an $\left(w_{0}, \alpha\right)$ violating partition of $S_{i}$ in graph $G$. We return this partition as the outcome of the algorithm. If $\mathcal{A}_{\mathrm{ARV}}$ returns a cut of sparsity at least $\alpha$ for each set $S_{i}$, for $1 \leq i \leq \ell$, then we are guaranteed that each such set has the $\alpha_{\mathrm{BW}}$-bandwidth property in $G^{*}$, and we have therefore constructed a good tree-of-sets system.

\section{EXTENSIONS}

The following theorem gives a slightly stronger version of Theorem 3.5, which we believe will be useful in designing approximation algorithms for maximum throughput routing problems such as all-or-nothing flow and disjoint paths in node capacitated graphs. For brevity, given a collection $\mathcal{S}$ of vertex subsets, we denote $V(\mathcal{S})=\bigcup_{S_{i} \in \mathcal{S}} S_{i}$.

THEOREM 6.1. There is a universal constant $\hat{c}>1$ and an efficient randomized algorithm that, given as input (i) a graph $G$ with maximum vertex degree $\Delta$, (ii) a subset $\mathcal{T}^{*}$ of $k^{*}$ vertices of $G$ called terminals that have degree 1 in $G$, such that $\mathcal{T}^{*}$ is $\alpha^{*}$-well-linked in G for some $0<\alpha^{*}<1$, and (iii) parameters $\ell^{*}, w^{*}>2$, such that $\frac{\alpha^{*} k^{*}}{\Delta^{23} \log ^{8.5} k^{*}}>\hat{c} w^{*}\left(\ell^{*}\right)^{48}$, with high probability computes the following:

- a subgraph $G^{*} \subseteq G$;

—a strong path-of-sets system $\left(\mathcal{S}^{*}, \bigcup_{i=1}^{\ell^{*}-1} \mathcal{P}_{i}, A_{1}, B_{\ell^{*}}\right)$ in $G^{*}$ with $\mathcal{T}^{*} \cap V\left(\mathcal{S}^{*}\right)=\emptyset$, such that every set $S \in \mathcal{S}^{*}$ has the $\alpha_{\mathrm{BW}}^{*}=\Omega\left(\frac{\left(\alpha^{*}\right)^{2}}{\Delta^{5}\left(\ell^{*}\right)^{16} \log ^{3} k^{*}}\right)$-bandwidth property in $G^{*}$; and 一 for each $S \in \mathcal{S}^{*}$, a set $\mathcal{Q}(S)$ of paths in $G^{*}$, connecting every terminal in $\mathcal{T}^{*}$ to some vertex of $S$, such that the paths in $\mathcal{Q}(S)$ cause edge congestion at most $\eta^{*}=O\left(\frac{\Delta^{5}\left(\ell^{*}\right)^{16} \log ^{3} k^{*}}{\left(\alpha^{*}\right)^{2}}\right)$.

We note that in approximation algorithms for routing problems, such as EdgeDisjoint Paths and Node-Disjoint paths, the typical setting of parameters is $\ell^{*}=$ $\Theta\left(\log ^{2} k\right)$ (roughly equal to the number of rounds in the cut-matching game), $\Delta=$ poly $\log k^{*}$, and $w^{*}=k^{*} /$ poly $\log k^{*}$, where $k^{*}$ is the number of terminals.

We briefly explain the relevance of the additional properties guaranteed by the preceding theorem for routing problems. A path-of-sets system can be used to embed an expander in $G^{*}$, and this in turn can be used as a crossbar routing structure; we refer the reader to prior work [Chekuri et al. 2005; Chuzhoy 2016; Chuzhoy and Li 2016] for more details on this approach. However, a technical issue that arises in using the crossbar for connecting the given input pairs is the following: we need to connect the input pairs to the interface of the crossbar. To avoid additional congestion in the routing, we 
would like the paths connecting the terminals to the interface to be disjoint from the crossbar itself. Theorem 6.1 helps in addressing this technical issue. We mention that the following simple approach does not work to yield the desired properties. We could start with a subgraph $G^{\prime}$ containing a path-of-sets system and then try to add paths from the terminals $\mathcal{T}^{*}$ to each $S \in \mathcal{S}^{*}$ by using the well-linkedness properties of the terminals. However, these paths may add new edges and alter the boundaries of the sets in $\mathcal{S}^{*}$, and hence a set $S \in \mathcal{S}^{*}$ that was previously boundary well-linked in $G^{\prime}$ may not have the property in the new subgraph $G^{\prime \prime}$ obtained from $G^{\prime}$ by adding the paths from the terminals to the path-of-sets system.

The remainder of this section is devoted to the proof of Theorem 6.1. Using Theorem 2.14, we compute a subset $\mathcal{T} \subseteq \mathcal{T}^{*}$ of $k=\left\lceil\frac{\alpha^{*} k^{*}}{32 \Delta^{4} \beta_{\operatorname{ARV}}\left(k^{*}\right)}\right\rceil$ terminals such that $\mathcal{T}$ is nodewell-linked in $G$. From the assumption in Theorem 6.1, $\frac{k}{\Delta^{19} \log ^{8} k} \geq \frac{k}{\Delta^{19} \log ^{8} k^{*}} \geq \hat{c} w^{*}\left(\ell^{*}\right)^{48}$ for some large enough constant $\hat{c}$. We set $\ell=3\left(\ell^{*}\right)^{2}+1$ and $w=\frac{\hat{c}}{2^{38 \cdot c}} \cdot w^{*}\left(\ell^{*}\right)^{10} \Delta^{11} \log ^{4} k$, so $w>4 \log k$ holds, where $c$ is the constant from Theorem 4.3. Clearly,

$$
c w \ell^{19} \Delta^{8} \leq\left(\frac{\hat{c}}{2^{38}} w^{*}\left(\ell^{*}\right)^{10} \Delta^{11} \log ^{4} k\right) \cdot\left(2 \ell^{*}\right)^{38} \Delta^{8}<\hat{c} \cdot w^{*}\left(\ell^{*}\right)^{48} \Delta^{19} \log ^{4} k \leq \frac{k}{\log ^{4} k}
$$

Therefore, $\frac{k}{\log ^{4} k}>c w \ell^{19} \Delta^{8}$, and the conditions of Theorem 4.3 hold for $G, \mathcal{T}$ and parameters $k, \ell$, and $w$. The main ingredient of the proof of Theorem 6.1 is the following generalization of Theorem 4.3 for the construction of a tree-of-sets system.

THEOREM 6.2. There is an efficient randomized algorithm that, given input as in Theorem 6.1, the set $\mathcal{T} \subseteq \mathcal{T}^{*}$ of terminals, and parameters $\ell, w, \alpha_{\mathrm{BW}}^{*}$ and $\eta^{*}$ as earlier, with high probability computes

— a subgraph $G^{*}$ of $G$;

-a tree-of-sets system $\left(\mathcal{S}, T, \cup_{e \in E(T)} \mathcal{P}_{e}\right)$ in $G^{*}$, with parameters $\left\lceil\frac{\ell-1}{3}\right\rceil, w$ and $\alpha_{\mathrm{BW}}=$ $\Omega\left(\frac{1}{\ell^{2} \log ^{1.5} k}\right)$, such that $\mathcal{T}^{*} \cap V(\mathcal{S})=\emptyset$, and every set $S \in \mathcal{S}$ has the $\alpha_{\mathrm{BW}}^{*}$-bandwidth property in $G^{*}$; and

- for each $S \in \mathcal{S}$, a set $\mathcal{Q}(S)$ of paths in $G^{*}$, connecting every terminal in $\mathcal{T}^{*}$ to some vertex of $S$, such that the paths in $\mathcal{Q}(S)$ cause edge congestion at most $\eta^{*}$.

Notice that the definition of the tree-of-sets system only requires that each set $S_{i} \in \mathcal{S}$ has the $\alpha_{\mathrm{BW}}$-bandwidth property in the subgraph of $G$ induced by the vertices of the tree-of-sets system. The preceding theorem requires a slightly stronger property, that additionally $S_{i}$ must have the $\alpha_{\mathrm{BW}}^{*}$-bandwidth property in the graph $G^{*}$ that contains both the tree-of-sets system and the set $\mathcal{Q}=\bigcup_{S \in \mathcal{S}} \mathcal{Q}(S)$ of paths.

We first complete the proof of Theorem 6.1 assuming Theorem 6.2, and then we provide a proof of the latter. This is done exactly as in the proof of Theorem 3.5, by first turning the tree-of-sets system into a strong one and then into a path-of-sets system. Consider the graph $G^{*}$, the tree-of-sets $\operatorname{system}\left(\mathcal{S}, T, \cup_{e \in E(T)} \mathcal{P}_{e}\right)$, and the sets of paths $\{\mathcal{Q}(S)\}_{S \in \mathcal{S}}$ returned by Theorem 6.2. As before, we use Lemma 4.5 to convert $\left(\mathcal{S}, T, \bigcup_{e \in E(T)} \mathcal{P}_{e}\right)$ into a strong tree-of-sets $\operatorname{system}\left(\mathcal{S}, T, \bigcup_{e \in E(T)} \mathcal{P}_{e}^{*}\right)$ with parameters $\ell$ and $\tilde{w}=\Omega\left(\frac{\alpha_{\mathrm{BW}}^{2}}{\Delta^{10}\left(\beta_{\mathrm{ARV}}(w)\right)^{2}} \cdot w\right)$ using Lemma 4.5. If $\hat{c}$ is chosen to be large enough, $\tilde{w}>16 w^{*}\left(\ell^{*}\right)^{2}+1$ must hold. We then apply Theorem 4.6 to obtain a path-of-sets 
system $\left(\mathcal{S}^{*}, \bigcup_{i=1}^{\ell^{*}-1} \mathcal{P}_{i}, A_{1}, B_{\ell^{*}}\right)$ with width $w^{*}$ and length $\ell^{*}$. Theorem 4.6 guarantees that $\mathcal{S}^{*} \subseteq \mathcal{S}$, and hence every set $S \in \mathcal{S}^{*}$ still has the $\alpha_{\mathrm{BW}}^{*}$-bandwidth property in $G^{*}$, and we can use the set $\mathcal{Q}(S)$ of paths computed for it by Theorem 6.2. It remains to prove Theorem 6.2.

Proof of Theorem 6.2. The proof closely follows the proof of Theorem 4.3, using the parameters $w, \ell, \alpha_{\mathrm{BW}}$, together with the set $\mathcal{T}$ of terminals. As before, if $\left(\mathcal{S}, T, \bigcup_{e \in E(T)} \mathcal{P}_{e}\right)$ is a tree-of-sets system in $G$, with parameters $w, \ell, \alpha_{\mathrm{BW}}$, and for each $S_{i} \in \mathcal{S}, S_{i} \cap \mathcal{T}=\emptyset$, then we say that it is a good tree-of-sets system (but we allow the sets in $\mathcal{S}$ to contain terminals of $\mathcal{T}^{*} \backslash \mathcal{T}$ ). We define the potential function, acceptable clustering, and good clustering exactly as before, using the parameters $w, \ell, \alpha_{\mathrm{BW}}$, and we set parameters $\ell_{0}, w_{0}, \alpha$ exactly as before, so $\ell_{0}=\ell^{2}$ and $w_{0}=\frac{k}{192 \ell_{0}^{3} \log k}$. Notice that under this definition of good clustering, the terminals of $\mathcal{T}^{*} \backslash \mathcal{T}$ are treated as regular vertices, and they do not necessarily reside in separate clusters. The algorithm again consists of several phases, where the input to every phase is a good clustering $\mathcal{C}$ of $V(G)$, and the output is either another good clustering $\mathcal{C}^{\prime}$ with $\varphi\left(\mathcal{C}^{\prime}\right) \leq \varphi(\mathcal{C})-1$ or a valid output for Theorem 6.2-that is, a subgraph $G^{*}$ of $G$, a tree-of-sets system $\left(\mathcal{S}, T, \bigcup_{e \in E(T)} \mathcal{P}_{e}\right)$ in $G^{*}$, and the sets $\mathcal{Q}(S)_{S \in \mathcal{S}}$ of paths as required. The initial clustering is defined exactly as before: $\{\{v\} \mid v \in V(G)\}$.

We now proceed to describe each phase. Suppose that the input to the current phase is a good clustering $\mathcal{C}$, and let $G^{\prime}$ be the corresponding legal contracted graph. We find the partition $\left\{X_{1}, \ldots, X_{\ell_{0}}\right\}$ of $V\left(G^{\prime}\right) \backslash \mathcal{T}$ and compute, for each $1 \leq j \leq \ell_{0}$, an acceptable clustering $\mathcal{C}_{j}$ exactly as before. Our only departure from the proof of Theorem 4.3 is that we replace Theorem 5.11 with the following theorem.

THEOREM 6.3. There is an efficient randomized algorithm that, given a collection $\left\{S_{1}, \ldots, S_{\ell_{0}}\right\}$ of disjoint vertex subsets of $G$, where for all $1 \leq j \leq \ell_{0}, S_{j} \cap \mathcal{T}=\emptyset$, with high probability computes one of the following:

- $a\left(w_{0}, \alpha\right)$-violating partition $(X, Y)$ of $S_{j}$, for some $1 \leq j \leq \ell_{0}$; or

- a partition $(A, B)$ of $V(G)$ with $S_{j} \subseteq A, \mathcal{T} \subseteq B$ and $\left|E_{G}(A, B)\right|<w_{0} / 2$, for some

$1 \leq j \leq \ell_{0}$; or

- a valid output for Theorem 6.2, that is

- a subgraph $G^{*}$ of $G$,

- a tree-of-sets system $\left(\mathcal{S}, T, \bigcup_{e \in E(T)} \mathcal{P}_{e}\right)$ in $G^{*}$, with parameters $\left\lceil\frac{\ell-1}{3}\right\rceil, w$ and $\alpha_{\mathrm{BW}}$, such that $\mathcal{T}^{*} \cap V(\mathcal{S})=\emptyset$, and every set $S \in \mathcal{S}$ has the $\alpha_{\mathrm{BW}}^{*}$-bandwidth property in $G^{*}$, and

- for each $S \in \mathcal{S}$, a set $\mathcal{Q}(S)$ of paths in $G^{*}$, connecting every terminal in $\mathcal{T}^{*}$ to some vertex of $S$, such that the paths in $\mathcal{Q}(S)$ cause edge congestion at most $\eta^{*}$.

Just as in the proof of Theorem 4.3, the proof of Theorem 6.2 follows from the proof of Theorem 6.3. We start with the initial collection $\mathcal{C}_{1}, \ldots, \mathcal{C}_{\ell_{0}}$ of acceptable clusterings, where for each $1 \leq j \leq \ell_{0}, \varphi\left(\mathcal{C}_{j}\right) \leq \varphi(\mathcal{C})-1$. If any of these clusterings $\mathcal{C}_{j}$ is a good clustering, then we terminate the phase and return this clustering. Otherwise, each clustering $\mathcal{C}_{j}$ must contain a large cluster $S_{j} \in \mathcal{C}_{j}$. We then iteratively apply Theorem 6.3 to clusters $\left\{S_{1}, \ldots, S_{\ell_{0}}\right\}$. If the outcome is a valid output for Theorem 6.2 , then we terminate the algorithm and return this output. Otherwise, we obtain either a $\left(w_{0}, \alpha\right)$-violating partition of some cluster $S_{j}$ or a partition $(A, B)$ of $V(G)$ with $S_{j} \subseteq A$, $\mathcal{T} \subseteq B$, and $\left|E_{G}(A, B)\right|<w_{0} / 2$, for some $1 \leq j \leq \ell_{0}$. We then apply the appropriate action: PARTITION $\left(S_{j}, X, Y\right)$ or $\operatorname{SEPARATE}\left(S_{j}, A\right)$ to the clustering $\mathcal{C}_{j}$ and obtain an acceptable clustering $\mathcal{C}_{j}^{\prime}$, with $\varphi\left(\mathcal{C}_{j}^{\prime}\right) \leq \varphi\left(\mathcal{C}_{j}\right)-1 / n$. If $\mathcal{C}_{j}^{\prime}$ is a good clustering, then we 
terminate the phase and return $\mathcal{C}_{j}^{\prime}$. Otherwise, we select an arbitrary large cluster $S_{j}^{\prime}$ in $\mathcal{C}_{j}^{\prime}$, replace $S_{j}$ with $S_{j}^{\prime}$, and continue to the next iteration. As before, we are guaranteed that after polynomially many iterations, the algorithm will terminate with the desired output.

From now on, we focus on proving Theorem 6.3. Given the input collection $\left\{S_{1}, \ldots, S_{\ell_{0}}\right\}$ of vertex subsets, we run the algorithm from Theorem 5.11 on it. If the outcome is a $\left(w_{0}, \alpha\right)$-violating partition $(X, Y)$ of $S_{j}$, for some $1 \leq j \leq \ell_{0}$, or a partition $(A, B)$ of $V(G)$ with $S_{j} \subseteq A, \mathcal{T} \subseteq B$ and $\left|E_{G}(A, B)\right|<w_{0} / 2$, for some $1 \leq j \leq \ell_{0}$, then we terminate the algorithm and return this partition.

Therefore, we can assume from now on that the algorithm from Theorem 5.11 has computed a good tree-of-sets system $\left(\mathcal{S}, T, \bigcup_{e \in E(T)} \mathcal{P}_{e}\right)$ in $G$, where $\mathcal{S}=\left\{S_{1}, \ldots, S_{\ell_{0}}\right\}$. Let $U=V(\mathcal{S})$. Recall that the algorithm also ensures that each set $S_{j}$ can send $w_{0} / 2$ flow units to the terminals of $\mathcal{T}$ with no edge congestion, as otherwise we could find a cut separating $S_{i}$ from $\mathcal{T}$ and containing fewer than $w_{0} / 2$ edges. Although we are guaranteed that $\mathcal{T} \cap U=\emptyset$, it is possible that some terminals of $\mathcal{T}^{*} \backslash \mathcal{T}$ belong to $U$-we take care of this issue later. The rest of the proof consists of three steps. In the first step, we construct the sets $\mathcal{Q}(S)$ of paths for all $S \in \mathcal{S}$, slightly alter the tree $T$ by discarding parts of it, and define the graph $G^{*}$. In the second step, we ensure that every set $S \in \mathcal{S}$ has the $\gamma$-bandwidth property in $G^{*}$ for a sufficiently large value $\gamma$. In the final step, we remove the vertices of $\mathcal{T}^{*} \backslash \mathcal{T}$ from the clusters $S$ and ensure that the resulting clusters have the $\alpha_{\mathrm{BW}}^{*}$-bandwidth property in $G^{*}$. If either of these steps fails, then we return a $\left(w_{0}, \alpha\right)$-violating partition of some cluster $S \in \mathcal{S}$.

Step 1: Finding the sets $\mathcal{Q}(S)$ of paths. The following lemma allows us to compute the sets $\mathcal{Q}(S)$ of paths.

Lemma 6.4. There is an efficient algorithm that either computes a $\left(w_{0}, \alpha\right)$-violating partition of some set $S^{\prime} \in \mathcal{S}$ or computes, for every set $S \in \mathcal{S}$, a collection $\mathcal{Q}(S)$ of paths, such that

—paths in $\mathcal{Q}(S)$ connect every terminal in $\mathcal{T}^{*}$ to some vertex of $S$, and they are internally disjoint from $\mathcal{T}^{*} \cup S$;

- paths in $\mathcal{Q}(S)$ cause edge congestion at most $\eta^{*}$ in $G$; and

- for each $S^{\prime} \in \mathcal{S}$, for every path $P \in \mathcal{Q}(S), P \cap G\left[S^{\prime}\right]$ has at most three connected components.

Proof. We fix some set $S \in \mathcal{S}$. Recall that there is a flow $F$ of value $w_{0} / 2$ from the terminals in $\mathcal{T}$ to the vertices of $S$ with edge congestion at most 1 , where each terminal sends at most one unit of flow. This implies, by converting the fractional flow into an integral flow, that there is a subset $\mathcal{T}_{0} \subseteq \mathcal{T}$ of at least $w_{0} / 2$ terminals, and a collection $\mathcal{Q}_{0}(S)$ of edge-disjoint paths, connecting $\mathcal{T}_{0}$ to $S$. We partition the terminals of $\mathcal{T}^{*} \backslash \mathcal{T}_{0}$ into $r \leq 4 k^{*} / w_{0}$ subsets $\mathcal{T}_{i}$, of cardinality at most $w_{0} / 2$ each. From the $\alpha^{*}$-welllinkedness of the terminals, for each such set $\mathcal{T}_{i}$, there is a collection $\mathcal{Q}_{i}(S)$ of paths, connecting $\mathcal{T}_{i}$ to $\mathcal{T}_{0}$ with edge congestion at most $1 / \alpha^{*}$.

For $i>0$, we view the paths in $\mathcal{Q}_{i}(S)$ as directed from the vertices of $\mathcal{T}_{i}$ to the vertices of $\mathcal{T}_{0}$, and we view the paths of $\mathcal{Q}_{0}(S)$ as directed from the vertices of $\mathcal{T}_{0}$ to the vertices of $S$. Fix some $0 \leq i \leq r$. We now reroute the paths in $\mathcal{Q}_{i}(S)$ to ensure that for each cluster $S^{\prime} \in \mathcal{S}$, the intersection of each such path with $G\left[S^{\prime}\right]$ has at most two connected components.

Consider some set $S^{\prime} \in \mathcal{S}$. Let $\mathcal{Q}_{i}\left(S, S^{\prime}\right) \subseteq \mathcal{Q}_{i}(S)$ be the following subset of paths: $P \in \mathcal{Q}_{i}\left(S, S^{\prime}\right)$, if $P \in \mathcal{Q}_{i}(S)$ and $P \cap G\left[S^{\prime}\right]$ has more than two connected components. For $P \in \mathcal{Q}_{i}\left(S, S^{\prime}\right)$, let $\Sigma(P)$ be the set of these connected components. We can order 
these components based on the orientation of $P$. Note that the first component of $\Sigma(P)$ may contain a terminal of $\mathcal{T}^{*} \backslash \mathcal{T}$ if it belongs to $S^{\prime}$. In such a case, we discard the corresponding component from $\Sigma(P)$. We denote by $s_{P}, t_{P}$ the first and the last vertices of $P$, respectively, that belong to any remaining component of $\Sigma(P)$; it can be seen that $s_{P}, t_{P} \in \Gamma_{G}\left(S^{\prime}\right)$. We will refer to $s_{P}$ and $t_{P}$ as the source and the destination vertex, respectively, of the pair $\left(s_{P}, t_{P}\right)$. Our goal is to find, for each $P \in \mathcal{Q}\left(S, S^{\prime}\right)$, an alternate path from $s_{p}$ to $t_{p}$ that is completely contained inside $G\left[S^{\prime}\right]$; we do this by exploiting the boundary well-linkedness properties of $S^{\prime}$. Let $\mathcal{M}_{i}\left(S^{\prime}\right)=\left\{\left(s_{P}, t_{P}\right) \mid P \in \mathcal{Q}_{i}\left(S, S^{\prime}\right)\right\}$; in fact, this is a multiset since $\left(s_{P}, t_{P}\right)$ can be the source and destination vertices for multiple paths $P$. Observe that $\left|\mathcal{M}_{i}\left(S^{\prime}\right)\right| \leq\left|\mathcal{Q}_{i}(S)\right| \leq w_{0} / 2$, and a vertex of $\Gamma_{G}\left(S^{\prime}\right)$ may belong to at most $\Delta / \alpha^{*}$ pairs in $\mathcal{M}_{i}\left(S^{\prime}\right)$ (since the paths in $\mathcal{Q}_{i}(S)$ cause vertexcongestion at most $\left.\Delta / \alpha^{*}\right)$. Let $\Gamma^{\prime} \subseteq \Gamma_{G}\left(S^{\prime}\right)$ contain all vertices that participate in the pairs in $\mathcal{M}_{i}\left(S^{\prime}\right)$, and hence $\left|\Gamma^{\prime}\right| \leq w_{0}$.

We use algorithm $\mathcal{A}_{\mathrm{ARV}}$ to approximately compute the sparsest cut $(A, B)$ of $G\left[S^{\prime}\right]$ with respect to the set $\Gamma^{\prime}$ of terminals. If the sparsity of the cut is at less than $\alpha$, then $(A, B)$ is a $\left(w_{0}, \alpha\right)$-violating partition of $S^{\prime}$. We then return this partition and terminate the algorithm. Otherwise, we are guaranteed that the vertices of $\Gamma^{\prime}$ are $\alpha_{\mathrm{BW}}$-well-linked in $G\left[S^{\prime}\right]$. Let $X=\left\{s_{P} \mid P \in \mathcal{Q}_{i}\left(S, S^{\prime}\right)\right\}$ and $Y=\left\{t_{P} \mid P \in \mathcal{Q}_{i}\left(S, S^{\prime}\right)\right\}$, where each set is a multiset-that is, a vertex $v$ that serves as a source vertex in $n_{v}$ pairs of $\mathcal{M}_{i}\left(S^{\prime}\right)$ appears $n_{v}$ times in $X$, and the same holds for vertices of $Y$.

OBSERVATION 6.5. There is a collection $\mathcal{R}$ of paths in $G\left[S^{\prime}\right]$, connecting vertices $X$ to vertices $Y$ with edge congestion at most $O\left(\frac{\Delta}{\alpha_{\mathrm{BW}} \cdot \alpha^{*}}\right)$, such that if $v$ appears $n_{v}$ times in $X$, then exactly $n_{v}$ paths of $\mathcal{R}$ originate at $v$, and if $v$ appears $n_{v}^{\prime}$ times in $Y$, then exactly $n_{v}^{\prime}$ paths of $\mathcal{R}$ terminate at $v$.

Proof. Since every vertex of $X \cup Y$ may participate in at most $\Delta / \alpha^{*}$ pairs in $\mathcal{M}_{i}\left(S^{\prime}\right)$, we can partition the set $\mathcal{M}_{i}\left(S^{\prime}\right)$ into at most $z=\left\lceil 2 \Delta / \alpha^{*}\right\rceil$ subsets $\mathcal{N}_{1}, \ldots, \mathcal{N}_{z}$ such that for all $1 \leq j \leq z$, every vertex of $X \cup Y$ participates in at most one pair in $\mathcal{N}_{j}$. For each $1 \leq \bar{j} \leq \bar{z}$, we then denote by $X_{j}$ and $Y_{j}$ the sets of all source and all destination vertices, respectively, of the pairs in $\mathcal{N}_{j}$. Since $S^{\prime}$ has the $\alpha_{\mathrm{BW}}$-bandwidth property, we are guaranteed that $X_{j} \cup Y_{j}$ is $\alpha_{\mathrm{BW}}$-well-linked in $G\left[S^{\prime}\right]$. Therefore, there is a set $\mathcal{R}_{j}$ of paths in $G\left[S^{\prime}\right]$, connecting every vertex of $X_{j}$ to a distinct vertex of $Y_{j}$, with edge congestion at most $1 / \alpha_{\mathrm{BW}}$. We then set $\mathcal{R}=\bigcup_{j} \mathcal{R}_{j}$.

For every path $P \in \mathcal{Q}_{i}\left(S, S^{\prime}\right)$, we discard the segment of the path between $s_{P}$ and $t_{P}$, obtaining two subpaths $P_{1}, P_{2}$ of $P$. We then use the paths in $\mathcal{R}$ to glue all path segments in $\left\{P_{1} \mid P \in \mathcal{Q}_{i}\left(S, S^{\prime}\right)\right\}$ and $\left\{P_{2} \mid P \in \mathcal{Q}_{i}\left(S, S^{\prime}\right)\right\}$, obtaining a new collection of paths, connecting each vertex of $\mathcal{T}_{i}$ to a distinct vertex of $\mathcal{T}_{0}$ (if $i=0$, then the paths connect every vertex of $\mathcal{T}_{0}$ to some vertex of $S$ ). These new paths then replace the paths of $\mathcal{Q}_{i}\left(S, S^{\prime}\right)$ in $\mathcal{Q}_{i}(S)$. Note that after rerouting, a terminal in $\mathcal{T}_{i}$ (if $i>0$ ) may not connect to the same terminal in $\mathcal{T}_{0}$ as it did previously.

Once we process every cluster $S^{\prime} \in \mathcal{S}$ in this fashion, the final set $\mathcal{Q}_{i}(S)$ of paths causes edge congestion at most $O\left(\frac{\Delta}{\alpha_{\mathrm{BW}} \cdot \alpha^{*}}\right)$ and has the property that for every $S^{\prime} \in \mathcal{S}$ and every path $P \in \mathcal{Q}_{i}(S), P \cap G\left[S^{\prime}\right]$ contains at most two connected components (if $P \in \mathcal{Q}_{0}\left(S^{\prime}\right)$, then $P \cap G\left[S^{\prime}\right]$ may contain at most one connected component, as $P$ originates from a vertex of $\mathcal{T}$, that cannot lie in $S^{\prime}$ ). In our final step, we take the union of all paths in $\bigcup_{i=1}^{r} \mathcal{Q}_{i}(S)$ and concatenate them with $r$ copies of the paths in $\mathcal{Q}_{0}(S)$. This final set of paths is denoted by $\mathcal{Q}(S)$. It is immediate to verify that the paths in $\mathcal{Q}(S)$ connect every vertex of $\mathcal{T}^{*}$ to a vertex of $S$, and for all $S^{\prime} \in \mathcal{S}$ and $P \in \mathcal{Q}(S), P \cap G\left[S^{\prime}\right]$ has at most three connected components. The congestion caused by the paths in $\mathcal{Q}(S)$ is 
bounded by

$$
\begin{aligned}
2 r \cdot O\left(\frac{\Delta}{\alpha_{\mathrm{BW}} \cdot \alpha^{*}}\right) & =O\left(\frac{k^{*} \cdot \Delta}{w_{0} \cdot \alpha_{\mathrm{BW}} \cdot \alpha^{*}}\right) \\
& =O\left(\frac{k^{*} \Delta \ell_{0}^{3} \log k}{k \cdot \alpha^{*}} \cdot \ell_{0} \log k \beta_{\mathrm{ARV}}(k)\right) \\
& =O\left(\frac{k^{*} \Delta \ell^{8} \log ^{2.5} k}{\alpha^{*}} \cdot \frac{\Delta^{4} \beta_{\mathrm{ARV}}\left(k^{*}\right)}{\alpha^{*} k^{*}}\right) \\
& =O\left(\frac{\Delta^{5} \ell^{8} \log ^{3} k^{*}}{\left(\alpha^{*}\right)^{2}}\right) \\
& =O\left(\frac{\Delta^{5}\left(\ell^{*}\right)^{16} \log ^{3} k^{*}}{\left(\alpha^{*}\right)^{2}}\right)=\eta^{*} .
\end{aligned}
$$

Let $\mathcal{S}=\left\{S_{1}, \ldots, S_{\ell}\right\}$, and let $V(T)=\left\{v_{1}, \ldots, v_{\ell}\right\}$, where $v_{i}$ is the vertex corresponding to the cluster $S_{i}$. For a cluster $S_{i}$, let $\mathcal{T}\left(S_{i}\right)=\mathcal{T}^{*} \cap S_{i}$, and let $i^{*}=\arg \max _{i}\left|\mathcal{T}\left(S_{i}\right)\right|$ be the index of the cluster containing the largest number of terminals. Let $T^{\prime}$ be the largest connected component of $T \backslash\left\{v_{i^{*}}\right\}$. Since $T$ has maximum degree $3,\left|V\left(T^{\prime}\right)\right| \geq\left\lceil\frac{\ell-1}{3}\right\rceil$. If $\left|V\left(T^{\prime}\right)\right|>\left\lceil\frac{\ell-1}{3}\right\rceil$, we discard leaves of $T^{\prime}$ until the equality holds. We discard from $\mathcal{S}$ all clusters except those corresponding to the vertices of $T^{\prime}$, obtaining a new tree-of-sets system $\left(T^{\prime}, \mathcal{S}, \bigcup_{e \in E\left(T^{\prime}\right)} \mathcal{P}(e)\right)$. For simplicity, we will denote $T^{\prime}$ by $T$ from now on. The new tree-of-sets system has the property that for each $S_{i} \in \mathcal{S},\left|\mathcal{T}\left(S_{i}\right)\right| \leq k^{*} / 2$. Since the terminals of $\mathcal{T}^{*}$ are $\alpha^{*}$-well-linked, there is a set $\mathcal{R}_{i}$ of paths in $G$, connecting every terminal in $\mathcal{T}\left(S_{i}\right)$ to some terminal of $\mathcal{T}^{*} \backslash \mathcal{T}\left(S_{i}\right)$ with edge congestion at most $1 / \alpha^{*}$. By appropriately truncating each path $P$ in $\mathcal{R}_{i}$, we can ensure that it terminates at a vertex $u_{P} \in \Gamma_{G}\left(S_{i}\right)$, and that $P \subseteq G\left[S_{i}\right]$. We denote by $R_{i} \subseteq \Gamma_{G}\left(S_{i}\right)$ the set of endpoints of the resulting paths in $\mathcal{R}_{i}$. Then $\left|R_{i}\right| \leq k^{*} / 2$, and every vertex in $R_{i}$ serves as an endpoint of at most $\Delta / \alpha^{*}$ paths in $\mathcal{R}_{i}$.

We are now ready to define the graph $G^{*}$. This graph is the union of all subgraphs $G\left[S_{i}\right]$ for $S_{i} \in \mathcal{S}$ and paths $\left(\bigcup_{e \in E(T)} \mathcal{P}(e)\right) \cup\left(\bigcup_{S_{i} \in \mathcal{S}} \mathcal{Q}\left(S_{i}\right)\right)$.

Step 2: Ensuring bandwidth property of clusters. Consider some cluster $S_{i} \in \mathcal{S}$, and recall that we have already defined a subset $R_{i} \subseteq \Gamma_{G}\left(S_{i}\right)$ of its vertices. We let $R_{i}^{\prime}=$ $\Gamma_{G^{*}}\left(S_{i}\right)$. Recall that $\left|R_{i}\right| \leq k^{*} / 2$ and that set $R_{i}^{\prime}$ contains, for each path $Q \in \bigcup_{S \in \mathcal{S}} \mathcal{Q}(S)$, at most six vertices of $Q$ (as $Q \cap G\left[S_{i}\right]$ contains at most three connected components). Set $R_{i}^{\prime}$ also contains at most $3 w$ vertices for the paths from $\bigcup_{e \in E(T)} \mathcal{P}_{e}$ that terminate in $S_{i}$. Let $\hat{R}_{i}=R_{i} \cup R_{i}^{\prime}$. Then,

$$
\left|\hat{R}_{i}\right| \leq \frac{k^{*}}{2}+6\left\lceil\frac{\ell-1}{3}\right\rceil \cdot \frac{w_{0}}{2}+3 w .
$$

Since $k^{*}=\Theta\left(\frac{\Delta^{4} k \beta_{\mathrm{ARV}}\left(k^{*}\right)}{\alpha^{*}}\right)=\Theta\left(\frac{w_{0} \Delta^{4} \ell^{6} \beta_{\mathrm{ARV}}\left(k^{*}\right) \log k}{\alpha^{*}}\right)=\Theta\left(\frac{w_{0} \Delta^{4} \ell^{6} \log ^{1.5} k^{*}}{\alpha^{*}}\right)$, and $w \leq k^{*}$, we get that $\left|\hat{R}_{i}\right|=w_{0} \cdot O\left(\frac{\Delta^{4} \ell^{6} \log ^{1.5} k^{*}}{\alpha^{*}}\right)$.

We let $\rho=\Theta\left(\frac{\Delta^{4} \ell^{6} \log ^{1.5} k^{*}}{\alpha^{*}}\right)$ so that $\left|\hat{R}_{i}\right| \leq w_{0} \rho$, and we denote $\gamma=\frac{\alpha}{\rho \beta_{\operatorname{ARV}}\left(k^{*}\right)}$. We use the following claim.

Claim 6.6. There is an efficient algorithm that, given a cluster $S_{i} \in \mathcal{S}$, either certifies that $\hat{R}_{i}$ is $\gamma$-well-linked in $G\left[S_{i}\right]$ or returns a $\left(w_{0}, \alpha\right)$-violating partition of $S_{i}$. 
Proof. We use algorithm $\mathcal{A}_{\mathrm{ARV}}$ to approximately compute the sparsest cut $(A, B)$ of $G\left[S_{i}\right]$ with respect to the set $\hat{R}_{i}$ of terminals. If the sparsity of the cut is at least $\gamma \cdot \beta_{\mathrm{ARV}}\left(k^{*}\right)$, then we are guaranteed that $S$ has the $\gamma$-bandwidth property in $G^{*}$. Assume now that the sparsity of the cut is less than $\gamma \cdot \beta_{\mathrm{ARV}}\left(k^{*}\right)$. We claim that in this case, cut $(A, B)$ is a $\left(w_{0}, \alpha\right)$-violating partition of $S$.

Indeed,

$$
\begin{aligned}
|E(A, B)| & <\gamma \cdot \beta_{\mathrm{ARV}}\left(k^{*}\right) \cdot \min \left\{\left|A \cap \hat{R}_{i}\right|,\left|B \cap \hat{R}_{i}\right|\right\} \\
& =\frac{\alpha}{\rho} \cdot \min \left\{\left|A \cap \hat{R}_{i}\right|,\left|B \cap \hat{R}_{i}\right|\right\} \\
& \leq \frac{\alpha\left|\hat{R}_{i}\right|}{2 \rho} \\
& \leq \frac{\alpha w_{0}}{2}
\end{aligned}
$$

In particular, this shows that $|E(A, B)|<\alpha \cdot \min \left\{\left|A \cap \hat{R}_{i}\right|,\left|B \cap \hat{R}_{i}\right|, w_{0} / 2\right\}$, so this is indeed a $\left(w_{0}, \alpha\right)$-violating partition of $S_{i}$.

If, for any set $S_{i} \in \mathcal{S}$, Claim 6.6 returns a $\left(w_{0}, \alpha\right)$-violating partition, then we terminate the algorithm and return this partition. Therefore, we assume from now on that for each set $S_{i}$, the vertex set $\hat{R}_{i}$ is $\gamma$-well-linked in $G\left[S_{i}\right]$.

So far, we have obtained a graph $G^{*} \subseteq G$, a tree-of sets system $\left(T, \mathcal{S}, \bigcup_{e \in E(T)} \mathcal{P}(e)\right)$, and the sets $\{\mathcal{Q}(S)\}_{S \in \mathcal{S}}$ of paths as required, but it is still possible that the terminals of $\mathcal{T}^{*} \backslash \mathcal{T}$ belong to the vertex sets $S \in \mathcal{S}$. We rectify this in our final step.

Step 3: Removing the terminals from the clusters. In this step, we define a new treeof-sets system by replacing every cluster $S \in \mathcal{S}$ with cluster $S^{\prime}=S \backslash \mathcal{T}^{*}$. Let $\mathcal{S}^{\prime}$ denote the resulting set of clusters. Recall that the terminals in $\mathcal{T}^{*}$ all have degree 1 in $G$, and hence they cannot participate in the paths $\bigcup_{e \in E(T)} \mathcal{P}(e)$. Therefore, $\left(\mathcal{S}^{\prime}, T, \bigcup_{e \in E(T)} \mathcal{P}(e)\right)$ remains a valid tree-of-sets system. Consider a cluster $S_{i}^{\prime} \in \mathcal{S}^{\prime}$, and let $\Gamma_{i}^{\prime} \subseteq S_{i}^{\prime}$ be the set of vertices serving as endpoints of the paths in $\mathcal{P}(e)$ for all edges $e \in E(\bar{T})$ incident to the vertex $v_{i} \in V(T)$ that corresponds to the set $S_{i}$. Since the vertices of $\mathcal{T}^{*} \cap S_{i}$ all have degree 1, their removal from $S_{i}$ does not affect the well-linkedness of the set $\Gamma_{i}^{\prime}$ of vertices, and hence $\Gamma_{i}^{\prime}$ remains $\alpha_{\mathrm{BW}}$-well-linked in $G\left[S_{i}^{\prime}\right]$. Similarly, the set $\hat{R}_{i}$ of vertices remains $\gamma$-well-linked in $G\left[S_{i}^{\prime}\right]$.

If some vertex $t \in \mathcal{T}^{*}$ originally belonged to some cluster $S_{i}$, then without loss of generality, its corresponding path in $\mathcal{Q}\left(S_{i}\right)$ contained a single vertex: the vertex $t$. We now replace this path with a path containing a single edge, connecting $t$ to its unique neighbor $u_{t}$, that must belong to $S_{i}^{\prime}$. Notice that vertex $u_{t}$ now belongs to the boundary of $S_{i}^{\prime}$ in $G^{*}$, even though it may not belong to the boundary of $S_{i}$. The resulting set $\mathcal{Q}\left(S_{i}\right)$ of paths still connects all vertices of $\mathcal{T}^{*}$ to the vertices of $S_{i}^{\prime}$ with edge congestion at most $\eta^{*}$, but now we need to prove that each resulting cluster $S_{i}^{\prime} \in \mathcal{S}^{\prime}$ has the $\alpha_{\mathrm{BW}}^{*}$-bandwidth property in $G^{*}$. The following claim will finish the proof of Theorem 6.3.

\section{Claim 6.7. Each set $S_{i}^{\prime} \in \mathcal{S}^{\prime}$ has the $\alpha_{\mathrm{BW}}^{*}$-bandwidth property in $G^{*}$.}

Proof. Let $\Gamma^{\prime}=\Gamma_{G^{*}}\left(S_{i}^{\prime}\right) \cup R_{i}$. It suffices to prove that $\Gamma^{\prime}$ is $\alpha_{\mathrm{BW}}^{*}$-well-linked in $G\left[S_{i}\right]$. Consider a partition $(A, B)$ of $S_{i}^{\prime}$. Let $Z_{A}=\Gamma^{\prime} \cap A$ and $Z_{B}=\Gamma^{\prime} \cap B$.

We partition the vertices of $Z_{A}$ into two subsets: $Z_{A}^{\prime}$ contains all vertices that belonged to $\hat{R}_{i}$, and $Z_{A}^{\prime \prime}$ contains all remaining vertices, so each vertex in $Z_{A}^{\prime \prime}$ is a neighbor of some 
terminal in $\mathcal{T}^{*} \cap S_{i}$. We define a partition of $Z_{B}$ into $Z_{B}^{\prime}$ and $Z_{B}^{\prime \prime}$ similarly. We now consider two cases.

Assume first that both $\left|Z_{A}^{\prime}\right| \geq \frac{\alpha^{*}\left|Z_{A}^{\prime \prime}\right|}{2 \Delta}$ and $\left|Z_{B}^{\prime}\right| \geq \frac{\alpha^{*}\left|Z_{B}^{\prime \prime}\right|}{2 \Delta}$. In this case, since $\hat{R}_{i}$ is $\gamma$-welllinked in $G\left[S_{i}\right]$ (from Claim 6.6), we get that

$$
|E(A, B)| \geq \gamma \min \left\{\left|Z_{A}^{\prime}\right|,\left|Z_{B}^{\prime}\right|\right\} \geq \frac{\alpha^{*} \gamma}{4 \Delta} \cdot \min \left\{\left|Z_{A}\right|,\left|Z_{B}\right|\right\} .
$$

Recall that $\gamma=\frac{\alpha}{\rho \beta_{\operatorname{ARV}}\left(k^{*}\right)}=\Theta\left(\frac{\alpha \alpha^{*}}{\Delta^{4} \ell^{6} \log ^{2} k^{*}}\right)$, whereas $\alpha=\Omega\left(\frac{1}{\ell^{2} \log k}\right)=\Omega\left(\frac{1}{\ell^{2} \log k^{*}}\right)$, and hence

$$
\begin{aligned}
|E(A, B)| & \geq \Omega\left(\frac{\alpha\left(\alpha^{*}\right)^{2}}{\Delta^{5} \ell^{6} \log ^{2} k^{*}}\right) \cdot \min \left\{\left|Z_{A}\right|,\left|Z_{B}\right|\right\} \\
& \geq \Omega\left(\frac{\left(\alpha^{*}\right)^{2}}{\Delta^{5} \ell^{8} \log ^{3} k^{*}}\right) \cdot \min \left\{\left|Z_{A}\right|,\left|Z_{B}\right|\right\} \\
& =\Omega\left(\frac{\left(\alpha^{*}\right)^{2}}{\Delta^{5}\left(\ell^{*}\right)^{16} \log ^{3} k^{*}}\right) \cdot \min \left\{\left|Z_{A}\right|,\left|Z_{B}\right|\right\} \\
& \geq \alpha_{\mathrm{BW}}^{*} \cdot \min \left\{\left|Z_{A}\right|,\left|Z_{B}\right|\right\} .
\end{aligned}
$$

Assume now that $\left|Z_{A}^{\prime}\right|<\frac{\alpha^{*}\left|Z_{A}^{\prime \prime}\right|}{2 \Delta}$. Let $\mathcal{T}^{\prime \prime} \subseteq \mathcal{T}^{*}$ be the set of terminals $t$, such that $t \in S_{i}$, and the unique neighbor of $t$ belongs to $\bar{Z}_{A}^{\prime \prime}$. Then every vertex in $Z_{A}^{\prime \prime}$ has a neighbor in $\mathcal{T}^{\prime \prime}$, and $\left|\mathcal{T}^{\prime \prime}\right| \geq\left|Z_{A}^{\prime \prime}\right| \geq 2 \Delta\left|Z_{A}^{\prime}\right| / \alpha^{*}$. Recall that we have defined a set $\mathcal{R}_{i}$ of paths in $G\left[S_{i}\right]$, connecting $\mathcal{T}^{*} \cap S_{i}$ to $R_{i} \subseteq \hat{R}_{i}$, with edge congestion at most $\alpha^{*} / \Delta$. Let $\mathcal{R}^{\prime} \subseteq \mathcal{R}_{i}$ be the set of paths originating at the vertices of $\mathcal{T}^{\prime \prime}$. Then at most half the paths in $\mathcal{R}^{\prime}$ may terminate at the vertices of $Z_{A}^{\prime}$, and each one of the remaining paths must contain an edge of $E(A, B)$. Since the paths cause edge congestion at most $\alpha^{*}$, we conclude that

$$
E(A, B) \geq \frac{\alpha^{*} \cdot\left|Z_{A}^{\prime \prime}\right|}{2} \geq \frac{\alpha^{*}}{4}\left|Z^{\prime \prime}(A)\right|+2 \Delta\left|Z_{A}^{\prime}\right| \geq \frac{\alpha^{*}}{4}|Z(A)|>\alpha_{\mathrm{BW}}^{*}|Z(A)| .
$$

The case where $\left|Z_{B}^{\prime}\right|<\frac{\alpha^{*}\left|Z_{B}^{\prime \prime}\right|}{2 \Delta}$ is analyzed similarly.

\section{APPENDIX}

\section{A. PROOFS OMITTED FROM SECTION 2}

\section{A.1. Proof of Theorem 2.14}

We start with a nonconstructive proof, as it is much simpler and gives better parameters. This proof can be turned into an algorithm whose running time is $\operatorname{poly}(n) \cdot 2^{\kappa}$. We then show a constructive proof with running time $\operatorname{poly}(n, \kappa)$.

A.1.1. A Nonconstructive Proof. A separation in graph $G$ is two subgraphs $Y, Z$ of $G$ such that every edge of $G$ belongs to exactly one of $Y, Z$, and $G=Y \cup Z$. The order of the separation is $|V(Y) \cap V(Z)|$. We say that a separation $(Y, Z)$ is balanced with respect to $\mathcal{T}$ if and only if $|V(Y) \cap \mathcal{T}|,|V(Z) \cap \mathcal{T}| \geq|\mathcal{T}| / 4$. Let $(Y, Z)$ be a balanced separation of $G$ with respect to $\mathcal{T}$ of minimum order, and let $X=V(Y) \cap V(Z)$. Assume without loss of generality that $|V(Y) \cap \mathcal{T}| \geq|V(Z) \cap \mathcal{T}|$, so $|V(Y) \cap \mathcal{T}| \geq|\mathcal{T}| / 2$. We claim that $X$ is node-well-linked in graph $Y$.

Claim A.1. Set $X$ of vertices is node-well-linked in graph $Y$.

Proof. Let $A, B$ be any two equal-size subsets of $X$, and assume that $|A|=|B|=z$. It is enough to show that there is a set $\mathcal{P}$ of $z$ disjoint paths connecting $A$ to $B$ in $Y$. Assume otherwise. Then there is a set $S$ of at most $z-1$ vertices separating $A$ from $B$ in $Y$. 
Let $\mathcal{C}$ be the set of all connected components of $Y \backslash S$. We partition $\mathcal{C}$ into three subsets: $\mathcal{C}_{1}$ contains all components containing the vertices of $A, \mathcal{C}_{2}$ contains all components containing the vertices of $B$, and $\mathcal{C}_{3}$ contains all remaining components (notice that all three sets of clusters are pairwise disjoint). Let $R_{1}=\bigcup_{C \in \mathcal{C}_{1}} V(C)$, and define $R_{2}$ and $R_{3}$ for $\mathcal{C}_{2}$ and $\mathcal{C}_{3}$, respectively. Assume without loss of generality that $\left|R_{1} \cap \mathcal{T}\right| \geq\left|R_{2} \cap \mathcal{T}\right|$. We define a new separation $\left(Y^{\prime}, Z^{\prime}\right)$ as follows. The set of vertices $V\left(Y^{\prime}\right)=R_{1} \cup R_{3} \cup S$, and $V\left(Z^{\prime}\right)=V(Z) \cup R_{2} \cup S$. Let $X^{\prime}=V\left(Y^{\prime}\right) \cap V\left(Z^{\prime}\right)$. The edges of $Y^{\prime}$ include all edges of $G$ with both endpoints in $V\left(Y^{\prime}\right) \backslash X^{\prime}$, and all edges of $G$ with one endpoint in $V\left(Y^{\prime}\right) \backslash X^{\prime}$ and the other endpoint in $X^{\prime}$. The edges of $Z^{\prime}$ include all edges with both endpoints in $Z^{\prime}$.

We claim that $\left(Y^{\prime}, Z^{\prime}\right)$ is a balanced separation with respect to $\mathcal{T}$. Clearly, $\left|V\left(Z^{\prime}\right) \cap \mathcal{T}\right| \geq$ $|\mathcal{T}| / 4$, since $V(Z) \subseteq V\left(Z^{\prime}\right)$, and $|V(Z) \cap \mathcal{T}| \geq|\mathcal{T}| / 4$. We next claim that $\mid V\left(Y^{\prime}\right) \cap$ $\mathcal{T}|\geq| \mathcal{T} \mid / 4$. Assume otherwise. Then, from our assumption, $\left|R_{2} \cap \mathcal{T}\right|<|\mathcal{T}| / 4$, and so $|V(\bar{Y}) \cap \mathcal{T}|=\left|R_{2} \cap \mathcal{T}\right|+\left|V\left(Y^{\prime}\right) \cap \mathcal{T}\right|<|\mathcal{T}| / 2$, a contradiction. Therefore, $\left(Y^{\prime}, Z^{\prime}\right)$ is a balanced separator with respect to $\mathcal{T}$. Finally, we claim that its order is less than $|X|$, contradicting the minimality of $X$. Indeed, $\left|V\left(Y^{\prime}\right) \cap V\left(Z^{\prime}\right)\right| \leq|X|-|B|+|S|<|X|$.

Let $\mathcal{T}_{1}=\mathcal{T} \cap V(Z), \mathcal{T}_{2}=\mathcal{T} \cap V(Y)$, and let $\mathcal{T}_{1}^{\prime} \subseteq \mathcal{T}_{1}, \mathcal{T}_{2}^{\prime} \subseteq \mathcal{T}_{2}$ be two disjoint subsets containing $\lceil\kappa / 4\rceil$ vertices each. From Observations 2.6 and 2.4, there is a flow $F$ from $\mathcal{T}_{1}^{\prime}$ to $\mathcal{T}_{2}^{\prime}$ such that every vertex in $\mathcal{T}_{1}^{\prime}$ sends one flow unit, every vertex in $\mathcal{T}_{2}^{\prime}$ receives one flow unit, and the congestion on every edge is at most $1 / \alpha$. We now bound the vertex congestion caused by the flow $F$. For every vertex $v \in V(G)$, let $F_{1}(v)$ be the total amount of flow on all paths that originate or terminate at $v$, and let $F_{2}(v)$ be the total amount of flow on all paths that contain $v$ as an inner vertex. It is immediate to verify that $F_{1}(v) \leq 1$, whereas $F_{2}(v) \leq \frac{\Delta}{2 \alpha}$, since every flow-path $P$ that contains $v$ as an inner vertex contributes flow $F(P)$ to two edges incident to $v$. Therefore, the total flow through $v$ is at most $\frac{\Delta}{2 \alpha}+1 \leq \frac{5 \Delta}{6 \alpha}$, as $\Delta \geq 3$ and $\alpha \leq 1$. By sending $\frac{6 \alpha}{5 \Delta} \cdot F(P)$ flow units via every path $P$, we obtain a flow of value at least $\frac{\kappa}{4} \cdot \frac{6 \alpha}{5 \Delta}=\frac{3 \alpha \kappa}{10 \Delta}$ from vertices of $\mathcal{T}_{1}^{\prime}$ to vertices of $\mathcal{T}_{2}^{\prime}$, which causes vertex congestion at most 1 . From the integrality of flow, there is a set $\mathcal{P}^{\prime}$ of $\kappa^{\prime}=\left\lceil\frac{3 \alpha \kappa}{10 \Delta}\right\rceil$ node-disjoint paths connecting terminals in $\mathcal{T}_{1}^{\prime}$ to terminals in $\mathcal{T}_{2}^{\prime}$ in $G$. Each such path has to contain a vertex of $X$. For each path $P^{\prime} \in \mathcal{P}^{\prime}$, we truncate the path $P^{\prime}$ to the first vertex of $X$ on $P^{\prime}$ (where the path is directed from $\mathcal{T}_{1}$ to $\mathcal{T}_{2}$ ). Let $\mathcal{P}$ be the resulting set of truncated paths. Then $\mathcal{P}$ is a set of $\kappa^{\prime}$ disjoint paths, connecting vertices of $\mathcal{T}_{1}^{\prime}$ to vertices of $X$; every path in $\mathcal{P}$ is completely contained in graph $Z$ and is disjoint from $X$ except for its last endpoint that belongs to $X$.

Let $\mathcal{T}^{\prime \prime} \subseteq \mathcal{T}_{1}^{\prime}$ be the set of terminals from which the paths in $\mathcal{P}$ originate, and let $X^{\prime} \subseteq X$ be the set of vertices where they terminate. We claim that $\mathcal{T}^{\prime \prime}$ is node-welllinked in $G$. Indeed, let $A, B \subseteq \mathcal{T}^{\prime \prime}$ be any pair of equal-size subsets of terminals. Let $U=A \cap B, A^{\prime}=A \backslash U$, and $B^{\prime}=B \backslash U$.

We define the set $\tilde{A}^{\prime} \subseteq X^{\prime}$ as follows: for each terminal $t \in A^{\prime}$, let $P_{t} \in \mathcal{P}$ be the path originating at $t$, and let $x_{t}$ be its other endpoint, which belongs to $X$. We then set $\tilde{A}^{\prime}=\left\{x_{t} \mid t \in A^{\prime}\right\}$. We define a set $\tilde{B}^{\prime} \subseteq X$ similarly for $B^{\prime}$. Let $\mathcal{P}_{A} \subseteq \mathcal{P}$ be the set of paths originating at the vertices of $A^{\prime}$, and let $\mathcal{P}_{B} \subseteq \mathcal{P}$ be the set of paths originating at the vertices of $B^{\prime}$. Notice that both sets of paths are contained in $Z$ and are internally disjoint from $X$. The paths in $\mathcal{P}_{A} \cup \mathcal{P}_{B}$ are also mutually disjoint, and they avoid $U$.

Let $U^{\prime}=U \cap X$, and consider the two subsets $\tilde{A}=\tilde{A}^{\prime} \cup U^{\prime}$ and $\tilde{B}=\tilde{B}^{\prime} \cup U^{\prime}$ of vertices of $X$. Denote $|\tilde{A}|=|\tilde{B}|=z$. Since $X$ is node-well-linked in $Y$, there is a set $\mathcal{Q}$ of $z$ disjoint paths connecting $\tilde{A}$ to $\tilde{B}$ in $Y$. The paths in $\mathcal{Q}$ are then completely disjoint from the paths in $\mathcal{P}_{1}, \mathcal{P}_{2}$ (except for sharing endpoints with them). The final set of paths connecting $A$ to $B$ is obtained by concatenating the paths in $\mathcal{P}_{1}, \mathcal{Q}, \mathcal{P}_{2}$, and adding a 
collection $\mathcal{Q}^{\prime}$ of paths that contains, for every vertex $v \in U \backslash U^{\prime}$, a path $P_{v}$ consisting of only the vertex $v$ itself.

A.1.2. A Constructive Proof. We assume that $\kappa \geq 32 \Delta^{4} \beta_{\mathrm{ARV}}(\kappa) / \alpha$, because otherwise we can return a set $\mathcal{T}^{\prime}$ consisting of a single terminal. For every subset $C \subseteq V$ of vertices, let $\mathcal{T}_{C}=C \cap \mathcal{T}$. We say that a partition $(A, B)$ of $V$ is balanced with respect to $\mathcal{T}$ if $\left|\mathcal{T}_{A}\right|,\left|\mathcal{T}_{B}\right| \geq \frac{\kappa}{2 \Delta}$. We need the following lemma that follows from the well-linkedness of $\mathcal{T}$.

Lemma A.2. Let $(A, B)$ be any balanced partition of $V$ with respect to $\mathcal{T}$. There is an efficient algorithm that computes a collection $\mathcal{P}$ of node-disjoint paths from $\mathcal{T}_{B}$ to $\mathcal{T}_{A}$ where $|\mathcal{P}| \geq\left\lceil\frac{\kappa \alpha}{2 \Delta^{2}}\right\rceil$.

Proof. Assume without loss of generality that $\left|\mathcal{T}_{B}\right| \leq\left|\mathcal{T}_{A}\right|$. Since $\mathcal{T}$ is $\alpha$-well-linked in $G$, from Observations 2.6 and 2.4 , there is a flow $F$ in $G$, where every vertex of $\mathcal{T}_{B}$ sends one flow unit, every vertex in $\mathcal{T}_{A}$ receives at most one flow unit, and the edge congestion is at most $1 / \alpha$. Therefore, the amount of flow through any vertex is at most $\Delta / \alpha$. Scaling this flow down by factor $\Delta / \alpha$, we obtain a $\mathcal{T}_{B}-\mathcal{T}_{A}$ flow of value at least $\frac{\kappa \alpha}{2 \Delta^{2}}$ and vertex congestion at most 1 . From the integrality of flow, there is a set of $\left\lceil\frac{k \alpha}{2 \Delta^{2}}\right\rceil$ disjoint paths connecting terminals in $\mathcal{T}_{B}$ to terminals in $\mathcal{T}_{A}$.

Suppose that we are given a balanced partition $(A, B)$ of $V$ with respect to $\mathcal{T}$ and a collection of paths $\mathcal{P}$ from $\mathcal{T}_{B}$ to $\mathcal{T}_{A}$. For each path $P \in \mathcal{P}$, let $v(P)$ be the first vertex of $P$ that lies in $A$, and let $\Gamma_{A}(\mathcal{P})=\left\{v_{P} \mid P \in \mathcal{P}\right\}$. We now show an algorithm to construct a balanced partition with some useful properties.

THEOREM A.3. There is an efficient algorithm to compute a balanced partition $(A, B)$ of $V$ with respect to $\mathcal{T}$ and a collection $\mathcal{P}$ of $\left\lceil\frac{\kappa \alpha}{2 \triangle^{2}}\right\rceil$ node-disjoint paths from $\mathcal{T}_{B}$ to $\mathcal{T}_{A}$ such that $G[B]$ is connected, and set $\Gamma_{A}(\mathcal{P})$ is $1 / \beta_{\mathrm{ARV}}(\kappa)$-well-linked in $G[A]$.

Proof. We say that a balanced partition $(A, B)$ of $V$ with respect to $\mathcal{T}$ is good if and only if both $G[A]$ and $G[B]$ are connected. We start with some initial good balanced partition $(A, B)$ and apply Lemma A.2 to find a collection of paths $\mathcal{P}$, and then we perform several iterations. In every iteration, we will either find a new good balanced partition $\left(A^{\prime}, B^{\prime}\right)$ with $\left|E\left(A^{\prime}, B^{\prime}\right)\right|<|E(A, B)|$ or establish that the current partition has the required properties (after possibly switching $A$ and $B$ ). In the former case, we continue to the next iteration, and in the latter case, we terminate the algorithm and return the current partition $(A, B)$ and the set $\mathcal{P}$ of paths. Clearly, after at most $|E|$ iterations, our algorithm is guaranteed to terminate with the desired output.

The initial partition $(A, B)$ is computed as follows. Let $T$ be any spanning tree of $G$, rooted at any vertex. Let $v$ be the lowest vertex of $T$ whose subtree contains at least $\frac{\kappa}{2 \Delta}$ terminals. Since the degree of every vertex is at most $\Delta$, the subtree of $T$ rooted at $v$ contains at most $\frac{\kappa}{2}+1$ terminals. We let $A$ contain all vertices in the subtree of $T$ rooted at $v$ (including $v$ ), and we let $B$ contain all remaining vertices. Then both $A$ and $B$ contain at least $\frac{\kappa}{2 \Delta}$ terminals, and both $G[A]$ and $G[B]$ are connected.

Given any good balanced partition $(A, B)$ of $V$, we perform an iteration as follows. Assume without loss of generality that $\left|\mathcal{T}_{A}\right| \geq\left|\mathcal{T}_{B}\right|$ (otherwise, we switch $A$ and $B$ ). First, we apply Lemma A.2 to find a collection $\mathcal{P}$ of $\left\lceil\frac{\kappa \alpha}{2 \Delta^{2}}\right\rceil$ disjoint paths from $\mathcal{T}_{B}$ to $\mathcal{T}_{A}$. Let $S=\Gamma_{A}(\mathcal{P})$; note that $|S| \leq \kappa / 2$. For a subset $Z \subseteq A$ of vertices, we denote $S_{Z}=Z \cap S$. We set up an instance of the sparsest cut problem in graph $G[A]$ with the set $S$ of terminals. Let $(X, Y)$ be the partition of $A$ returned by the algorithm $\mathcal{A}_{\mathrm{ARV}}$ on this instance. If $\frac{|E(X, Y)|}{\min \left\{\left|S_{X}\right|,\left|S_{Y}\right|\right\}} \geq 1$, then we are guaranteed that $S$ is $1 / \beta_{\mathrm{ARV}}(\kappa)$-well-linked in 
$G[A]$. We then return $(A, B)$ and $\mathcal{P}$, which are guaranteed to satisfy the requirements of the theorem. We now assume that $\frac{|E(X, Y)|}{\min \left\{\left|S_{X}\right|,\left|S_{Y}\right|\right\}}=\rho<1$.

Our next step is to show that there is a partition $\left(X^{\prime}, Y^{\prime}\right)$ of $A$, such that $G\left[X^{\prime}\right]$ and $G\left[Y^{\prime}\right]$ are both connected, and the sparsity of the cut $\left(X^{\prime}, Y^{\prime}\right)$ in $G[A]$ (with respect to $S$ ) is at most $\rho$. To show this, we start with the cut $(X, Y)$ and perform several iterations. Let $\mathcal{C}$ be the set of all connected components of $G[A] \backslash E(X, Y)$. Each iteration will reduce the number of the connected components in $\mathcal{C}$ by at least 1 while preserving the sparsity of the cut. Let $\mathcal{C}_{1} \subseteq \mathcal{C}$ be the set of all connected components contained in $X$, and let $\mathcal{C}_{2} \subseteq \mathcal{C}$ be the set of connected components contained in $Y$. Assume without loss of generality that $\left|S_{X}\right| \leq\left|S_{Y}\right|$. If there is some component $C \in \mathcal{C}$ with $\left|S_{C}\right|=0$, then we can move the vertices of $C$ to the opposite side of the partition $(X, Y)$, and obtain a new partition $\left(X^{\prime}, Y^{\prime}\right)$ whose sparsity is less than $\rho$, and the number of connected components in $G[A] \backslash E\left(X^{\prime}, Y^{\prime}\right)$ is strictly smaller than $|\mathcal{C}|$. Therefore, we assume from now on that for each $C \in \mathcal{C},\left|S_{C}\right|>0$.

Assume first that $\left|\mathcal{C}_{1}\right|>1$. Then $|E(X, Y)|=\rho \cdot\left|S_{X}\right|$, and so there is a connected component $C \in \mathcal{C}_{1}$ with $|E(C, Y)| \geq \rho \cdot\left|S_{C}\right|$. Moreover, $\left|S_{X}\right|>\left|S_{C}\right|$, as we have assumed that for each $C^{\prime} \in \mathcal{C},\left|S_{C^{\prime}}\right|>0$. Consider a new partition $\left(X^{\prime}, Y^{\prime}\right)$ of $A$, with $X^{\prime}=X \backslash C$ and $Y^{\prime}=Y \cup C$. Notice that the number of the connected components in $G[A] \backslash E\left(X^{\prime}, Y^{\prime}\right)$ is strictly smaller than $|\mathcal{C}|$. We claim that the sparsity of the new cut is at most $\rho$. Indeed, the sparsity of the new cut is

$$
\frac{\left|E\left(X^{\prime}, Y^{\prime}\right)\right|}{\left|S_{X^{\prime}}\right|}=\frac{|E(X, Y)|-|E(C, Y)|}{\left|S_{X}\right|-\left|S_{C}\right|} \leq \frac{\rho\left|S_{X}\right|-\rho\left|S_{C}\right|}{\left|S_{X}\right|-\left|S_{C}\right|}=\rho .
$$

Assume now that $\left|\mathcal{C}_{2}\right|>1$, and denote $|E(X, Y)| /\left|S_{Y}\right|=\rho^{\prime}$. Then $\rho^{\prime} \leq \rho$. As before, there is a connected component $C \in \mathcal{C}_{2}$ with $|E(C, X)| \geq \rho^{\prime}\left|S_{C}\right|$ and $\left|S_{C}\right|<\left|S_{Y}\right|$. Consider a new partition $\left(X^{\prime}, Y^{\prime}\right)$ of $A$, where $X^{\prime}=X \cup C$ and $\bar{Y}^{\prime}=Y \backslash C$. As before, the number of connected components in $G[A] \backslash E\left(X^{\prime}, Y^{\prime}\right)$ is strictly smaller than $|\mathcal{C}|$. We now show that the sparsity of the new cut is at most $\rho$. If $\left|S_{Y^{\prime}}\right| \leq\left|S_{X^{\prime}}\right|$, then the sparsity of the new cut is

$$
\frac{\left|E\left(X^{\prime}, Y^{\prime}\right)\right|}{\left|S_{Y^{\prime}}\right|}=\frac{|E(X, Y)|-|E(C, X)|}{\left|S_{Y}\right|-\left|S_{C}\right|} \leq \frac{\rho^{\prime}\left|S_{Y}\right|-\rho^{\prime}\left|S_{C}\right|}{\left|S_{X}\right|-\left|S_{C}\right|}=\rho^{\prime} \leq \rho .
$$

Otherwise, $\left|S_{X^{\prime}}\right|<\left|S_{Y^{\prime}}\right|$, and the sparsity of the new cut is

$$
\frac{\left|E\left(X^{\prime}, Y^{\prime}\right)\right|}{\left|S_{X^{\prime}}\right|}=\frac{|E(X, Y)|-|E(X, C)|}{\left|S_{X}\right|+\left|S_{C}\right|}<\frac{|E(X, Y)|}{\left|S_{X}\right|}=\rho .
$$

We continue this procedure until $|\mathcal{C}|=2$ holds, so in the end, $\mathcal{C}=\{G[X], G[Y]\}$, and the sparsity of the cut $(X, Y)$ is at most $\rho<1$. Assume without loss of generality that $\left|X \cap \mathcal{T}_{A}\right| \leq\left|Y \cap \mathcal{T}_{A}\right|$. We obtain a new partition $\left(A^{\prime}, B^{\prime}\right)$ of $V$ by setting $A^{\prime}=A \backslash X$ and $B^{\prime}=B \cup X$. Notice that this is guaranteed to be a balanced partition with respect to $\mathcal{T}$, as $\left|\mathcal{T}_{A}\right| \geq \kappa / 2$. The number of the edges in the new cut, $\left|E\left(A^{\prime}, B^{\prime}\right)\right| \leq|E(A, B)|-$ $|E(X, B)|+|E(X, Y)| \leq|E(A, B)|-\left|S_{X}\right|+|E(X, Y)|<|E(A, B)|$, since $|E(\bar{X}, Y)|<\left|S_{X}\right|$. Moreover, since $X$ contains at least one vertex of $S, G[B \cup X]$ is connected, and we established previously that $G[Y]$ is also connected. We then replace the partition $(A, B)$ with $\left(A^{\prime}, B^{\prime}\right)$ and continue to the next iteration.

We apply the preceding theorem to find a balanced partition $(A, B)$ of $V$ with respect to $\mathcal{T}$ and a corresponding collection $\mathcal{P}^{\prime}$ of paths.

Let $\Gamma \subseteq A$ be the set of vertices of $A$ incident to the edges of $E(A, B)$, and similarly let $\Gamma^{\prime} \subseteq \bar{B}$ be the set of vertices of $B$ incident to the edges of $E(A, B)$. Consider some path $\overline{P^{\prime}} \in \mathcal{P}^{\prime}$, and let $e=(v, u)$ be the first edge of $P^{\prime}$ that belongs to $E(A, B)$, with $v \in \Gamma^{\prime}, u \in \Gamma$. (Recall that the paths are directed from $\mathcal{T}_{B}$ to $\mathcal{T}_{A}$.) We truncate the path 
$P^{\prime}$ at vertex $v$, and we say that $u$ is a special neighbor of $v$. Let $\mathcal{P}$ be the resulting set of truncated paths. Then $\mathcal{P}$ is a set of $\left\lceil\frac{\kappa \alpha}{2 \Delta^{2}}\right\rceil$ node-disjoint paths, connecting the vertices of $\mathcal{T}_{B}$ to the vertices of $\Gamma^{\prime}$, and every path in $\mathcal{P}$ is completely contained in $G[B]$. Moreover, if $\Gamma^{\prime \prime} \subseteq \Gamma^{\prime}$ denotes the set of endpoints of the paths in $\mathcal{P}$, then for each $v \in \Gamma^{\prime \prime}$, we have defined a special neighbor $u \in \Gamma$ such that if $v \neq v^{\prime} \in \Gamma^{\prime \prime}$, then their special neighbors are distinct. Let $S$ be the set of the special neighbors. Recall that from Theorem A.3, $S$ is $1 / \beta_{\mathrm{ARV}}(\kappa)$-well-linked in $G[A]$. Let $q=2 \Delta \beta_{\mathrm{ARV}}(\kappa)$. We need the following theorem, which allows us to group the paths in $\mathcal{P}$ inside the graph $G[B]$.

THeOREM A.4. There is an efficient algorithm to find a subset $\tilde{\mathcal{P}} \subseteq \mathcal{P}$ of at least $|\mathcal{P}| / 2$ paths, and a collection $\mathcal{C}$ of disjoint connected subgraphs of $G[B]$, such that each path $P \in \tilde{\mathcal{P}}$ is completely contained in some subgraph $C \in \mathcal{C}$, and each such subgraph contains at least $q$ and at most $4 \Delta q$ paths in $\tilde{\mathcal{P}}$.

Proof. Start from $G[B]$ and build a new graph $H$ by contracting every path $P \in \mathcal{P}$ into a supernode $u_{P}$. Let $U=\left\{u_{P} \mid P \in \mathcal{P}\right\}$ be the resulting set of supernodes. Let $T$ be any spanning tree of $H$, rooted at any vertex $r$. Given a vertex $v \in V(T)$, let $T_{v}$ be the subtree of $T$ rooted at $v$. Let $J_{v} \subseteq V(G)$ be the set of all vertices of $T_{v}$ that do not belong to $U$ and all vertices on paths $P$ with $u_{P} \in T_{v}$. In other words, $J_{v}=\left(V\left(T_{v}\right) \backslash U\right) \cup\left(\bigcup_{u_{P} \in V\left(T_{v}\right) \cap U} V(P)\right)$. Denote $G_{v}=G\left[J_{v}\right]$. Over the course of the algorithm, we will delete some vertices of $T$. The notations $T_{v}$ and $G_{v}$ are always computed with respect to the most current tree $T$. We start with $\mathcal{C}=\emptyset, \tilde{\mathcal{P}}=\emptyset$ and then iterate.

Each iteration is performed as follows. If $q \leq|V(T) \cap U| \leq 4 \Delta q$, then we add $G_{r}$ to $\mathcal{C}$ and terminate the algorithm. If $|V(T) \cap U|<q$, then we also terminate the algorithm (we will show later that $\tilde{\mathcal{P}}$ must contain at least $|\mathcal{P}| / 2$ paths at this point). Otherwise, let $v$ be the lowest vertex of $T$ with $\left|T_{v} \cap U\right| \geq q$. If $v \notin U$, then, since the degree of every vertex is at most $\Delta,\left|T_{v} \cap U\right| \leq \Delta q$. We add $G_{v}$ to $\mathcal{C}$ and all paths in $\left\{P \mid u_{P} \in T_{v}\right\}$ to $\tilde{\mathcal{P}}$. We then delete all vertices of $\bar{T}_{v}$ from $T$ and continue to the next iteration.

Assume now that $v=u_{P}$ for some path $P \in \mathcal{P}$. If $\left|T_{v} \cap U\right| \leq 4 \Delta q$, then we add $G_{v}$ to $\mathcal{C}$, and all paths in $\left\{P^{\prime} \mid u_{P^{\prime}} \in T_{v}\right\}$ to $\tilde{\mathcal{P}}$, and continue to the next iteration. Thus, we assume that $\left|T_{v} \cap U\right|>4 \Delta q$.

Let $v_{1}, \ldots, v_{z}$ be the children of $v$ in $T$. Build a new tree $T^{\prime}$ as follows. Start with the path $P$, and add the vertices $v_{1}, \ldots, v_{z}$ to $T^{\prime}$. For each $1 \leq i \leq z$, let $\left(x_{i}, y_{i}\right) \in E(G[B])$ be any edge connecting some vertex $x_{i} \in V(P)$ to some vertex $y_{i} \in V\left(G_{v_{i}}\right)$; such an edge must exist from the definition of $G_{v_{i}}$ and $T$. Add the edge $\left(v_{i}, x_{i}\right)$ to $T^{\prime}$. Therefore, $T^{\prime}$ is the union of the path $P$, and a number of disjoint stars whose centers lie on the path $P$, and whose leaves are the vertices $v_{1}, \ldots, v_{z}$. The degree of every vertex of $P$ is at most $\Delta$. The weight of the vertex $v_{i}$ is defined to be the number of paths in $\mathcal{P}$ contained in $G_{v_{i}}$. Recall that the weight of each vertex $v_{i}$ is at most $q$, by the choice of $v$. For each vertex $x \in P$, the weight of $x$ is the total weight of its children in $T^{\prime}$. Recall that the total weight of the vertices of $P$ is at least $4 \Delta q$, and the weight of every vertex is at most $\Delta q$. We partition $P$ into several disjoint segments $\Sigma=\left(\sigma_{1}, \ldots, \sigma_{\ell}\right)$ of weight at least $q$ and at most $2 \Delta q$ each, as follows. Start with $\Sigma=\emptyset$ and then iterate. If the total weight of the vertices of $P$ is at most $2 \Delta q$, we build a single segment, containing the whole path. Otherwise, find the shortest segment $\sigma$ starting from the first vertex of $P$, whose weight is at least $q$. Since the weight of every vertex is at most $\Delta q$, the weight of $\sigma$ is at most $\Delta q$. We then add $\sigma$ to $\Sigma$, delete it from $P$, and continue. Consider the final set $\Sigma$ of segments. For each segment $\sigma$, we add a new graph $C_{\sigma}$ to $\mathcal{C}$. Graph $C_{\sigma}$ consists of the union of $\sigma$, the graphs $G_{v_{i}}$ for each $v_{i}$ that is connected to a vertex of $\sigma$ with an edge in $T^{\prime}$, and the corresponding edge $\left(x_{i}, y_{i}\right)$. Clearly, $C_{\sigma}$ is a connected subgraph of $G[B]$, containing at least $q$ and at most $2 \Delta q$ paths of $\mathcal{P}$. We add all of those paths to $\tilde{\mathcal{P}}$, 
delete all vertices of $T_{v}$ from $T$, and continue to the next iteration. We note that path $P$ itself is not added to $\tilde{\mathcal{P}}$, but all paths $P^{\prime}$ with $u_{P^{\prime}} \in V\left(T_{v}\right)$ are added to $\tilde{\mathcal{P}}$.

At the end of this procedure, we obtain a collection $\tilde{\mathcal{P}}$ of paths, and a collection $\mathcal{C}$ of disjoint connected subgraphs of $G$, such that each path $P \in \tilde{\mathcal{P}}$ is contained in some $C \in \mathcal{C}$, and each $C \in \mathcal{C}$ contains at least $q$ and at most $4 \Delta q$ paths from $\tilde{\mathcal{P}}$. It now remains to show that $|\tilde{\mathcal{P}}| \geq|\mathcal{P}| / 2$. We discard at most $q$ paths in the last iteration of the algorithm. Additionally, when $v=u_{P}$ is processed, if $\left|T_{v} \cap U\right|>4 \Delta q$, then path $P$ is also discarded, but at least $4 \Delta q$ paths are added to $\tilde{\mathcal{P}}$. Therefore, overall, $|\tilde{\mathcal{P}}| \geq|\mathcal{P}|-\frac{|\mathcal{P}|}{4 \Delta q+1}-q \geq|\mathcal{P}| / 2$, since $|\mathcal{P}|=\left\lceil\frac{\kappa \alpha}{2 \Delta^{2}}\right\rceil$, whereas $q=2 \Delta \beta_{\mathrm{ARV}}(\kappa)$, and we have assumed that $\kappa \geq \frac{32 \Delta^{4} \beta_{\mathrm{ARV}}(\kappa)}{\alpha}$.

For each graph $C \in \mathcal{C}$, we select one path $P_{C} \in \tilde{\mathcal{P}}$ that is contained in $C$, and we let $t_{C}$ be the terminal that serves as an endpoint of $P_{C}$. Let $\Gamma_{C}^{\prime} \subseteq \Gamma^{\prime}$ be the set of all vertices of $\Gamma^{\prime}$ that serve as endpoints of paths of $\tilde{\mathcal{P}}$ that are contained in $C$. Then $\left|\Gamma_{C}^{\prime}\right| \geq q$. We delete vertices from $\Gamma_{C}^{\prime}$ as necessary until $\left|\Gamma_{C}^{\prime}\right|=q$ holds. Our final set $\mathcal{T}^{\prime}$ of terminals is $\mathcal{T}^{\prime}=\left\{t_{C} \mid C \in \mathcal{C}\right\}$. Observe that $\left|\mathcal{T}^{\prime}\right| \geq \frac{|\tilde{\mathcal{P}}|}{4 \Delta q} \geq \frac{|\mathcal{P}|}{16 \Delta^{2} \beta_{\mathrm{ARV}}(\kappa)} \geq \frac{\kappa \alpha}{32 \Delta^{4} \beta_{\mathrm{ARV}}(\kappa)}$, as required.

It now only remains to show that $\mathcal{T}^{\prime}$ is node-well-linked in $G$. Let $\mathcal{T}_{1}$, $\mathcal{T}_{2}$ be any pair of equal-size subsets of $\mathcal{T}^{\prime}$. Let $\mathcal{T}^{*}=\mathcal{T}_{1} \cap \mathcal{T}_{2}, \mathcal{T}_{1}^{\prime}=\mathcal{T}_{1} \backslash \mathcal{T}_{2}$ and $\mathcal{T}_{2}^{\prime}=\mathcal{T}_{2} \backslash \mathcal{T}_{1}$. We set up an $s$ - $t$ flow network by adding a source $s$ and connecting it to every vertex of $\mathcal{T}_{1}^{\prime}$ with a directed edge, and adding a sink $t$, and connecting every vertex of $\mathcal{T}_{2}^{\prime}$ to it. We also delete all vertices of $\mathcal{T}^{*}$ from the graph and set all vertex capacities, except for $s$ and $t$, to 1 ; the capacities of $s$ and $t$ are infinite. From the integrality of flow, it is enough to show a valid $s$ - $t$ flow of value $\left|\mathcal{T}_{1}^{\prime}\right|=\left|\mathcal{T}_{2}^{\prime}\right|$ in this flow network. This flow will be a concatenation of three flows: $F_{1}, F_{2}, F_{3}$.

We start by defining the flows $F_{1}$ and $F_{3}$. Consider some terminal $t^{\prime} \in \mathcal{T}_{1}^{\prime} \cup \mathcal{T}_{2}^{\prime}$, and let $C \in \mathcal{C}$ be the subgraph to which $t^{\prime}$ belongs. Let $T_{C}$ be any spanning tree of $C$. Terminal $t^{\prime}$ sends one flow unit toward the vertices of $\Gamma_{C}^{\prime}$ along the tree $T_{C}$ such that every vertex in $\Gamma_{C}^{\prime}$ receives $1 / q$ flow units. Let $F_{1}$ be the union of all of these flows for all $t^{\prime} \in \mathcal{T}_{1}^{\prime}$, and let $F_{3}$ be the union of all of these flows for all $t^{\prime} \in \mathcal{T}_{2}^{\prime}$ (we will eventually think of the flow in $F_{3}$ as directed toward the terminals). Notice that for every vertex $v \in B \backslash \mathcal{T}^{*}$, the total flow that goes through vertex $v$ or terminates at $v$ is at most 1 . We say that the flow is of type 1 if it originates at a terminal in $\mathcal{T}_{1}^{\prime}$, and it is of type 2 otherwise.

We now proceed to define flow $F_{2}$. For every cluster $C \in \mathcal{C}$, each vertex $v \in \Gamma_{C}^{\prime}$ sends the $1 / q$ flow units it receives to its special neighbor $u \in \Gamma$, along the edge $(v, u)$. Recall that every vertex $u \in \Gamma$ serves as a special neighbor of at most one vertex in $\Gamma^{\prime}$. Let $\Gamma_{1} \subseteq \Gamma$ be the set of vertices that receive flow of type 1 , and $\Gamma_{2} \subseteq \Gamma$ is the set of vertices that receive flow of type 2 . Then $\left|\Gamma_{1}\right|=\left|\Gamma_{2}\right|$, and we denote $\left|\Gamma_{1}\right|=\kappa^{*}$. It is enough to show that there is a flow $F_{2}$ in $G[A]$, where every vertex in $\Gamma_{1}$ sends $1 / q$ flow units, every vertex in $\Gamma_{2}$ receives $1 / q$ flow units, and the total vertex congestion due to this flow is at most $1 / 2$.

To define this flow, recall that since $\Gamma_{1} \cup \Gamma_{2} \subseteq S$ and $S$ is $1 / \beta_{\mathrm{ARV}}(\kappa)$-well-linked in $G[A]$, from Observations 2.6 and 2.4 , there is a flow in $G[A]$, where every vertex in $\mathcal{T}_{1}$ sends one flow unit, every vertex in $\mathcal{T}_{2}$ receives one flow unit, and the edge congestion is bounded by $\beta_{\mathrm{ARV}}(\kappa)$. The total flow through every vertex is then at most $\Delta \beta_{\mathrm{ARV}}(\kappa)$. Scaling this flow down by factor $q=2 \Delta \beta_{\mathrm{ARV}}(\kappa)$, we obtain the flow $F_{2}$, where every vertex of $\Gamma_{1}$ sends $1 / q$ flow units, every vertex in $\Gamma_{2}$ receives $1 / q$ flow units, and the total vertex congestion is at most $1 / 2$. Combining together the flows $F_{1}, F_{2}, F_{3}$, we obtain the final flow $F$. From the integrality of flow, there is a set of $\left|\mathcal{T}_{1}\right|=\left|\mathcal{T}_{2}\right|$ disjoint paths connecting the vertices of $\mathcal{T}_{1}$ to the vertices of $\mathcal{T}_{2}$ in $G$. 

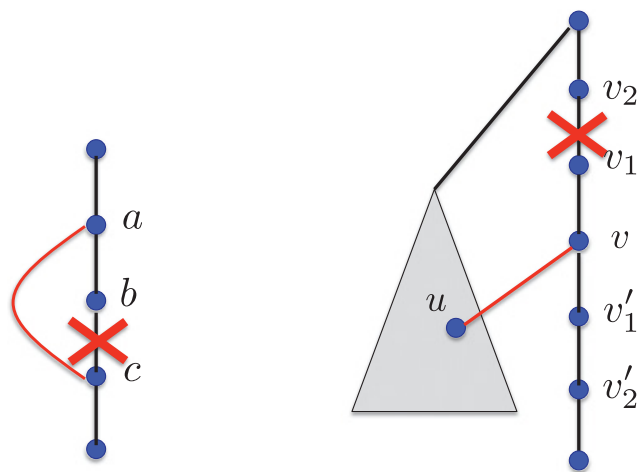

(b) Case 1

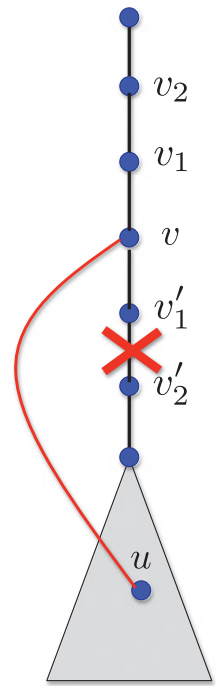

(c) Case 2

Fig. 5. Improvement steps to increase the number of leaves.

\section{A.2. Proof of Theorem 2.18}

Let $T$ be any spanning tree of the graph $Z$. If $T$ contains at least $L$ leaves, then we are done. Assume now that $T$ contains fewer than $L$ leaves. We will next try to perform some improvement steps to increase the number of leaves in $T$.

Assume first that $T$ contains three vertices $a, b, c$, which have degree 2 in $T$ each, where $b$ is the unique child of $a$ and $c$ is the unique child of $b$, and assume further that there is an edge $(a, c)$ in $Z$. We can then delete the edge $(b, c)$ and add the edge $(a, c)$ to $T$. It is easy to see that the number of leaves increases, with the new leaf being $b$ (Figure 5).

Assume now that $v$ is a degree- 2 vertex in $T$ such that both its father $v_{1}$ and grandfather $v_{2}$ are degree- 2 vertices. Moreover, assume that the unique child $v_{1}^{\prime}$ of $v$ is a degree-2 vertex, and so is the unique grandchild $v_{2}^{\prime}$ of $v$. Assume that an edge $(v, u)$ belongs to $Z$, where $u \neq v_{1}, v_{1}^{\prime}$. Notice that if $u=v_{2}$ or $u=v_{2}^{\prime}$, then we can apply the transformation outlined previously. Therefore, we assume that $u \neq v_{2}$ and $u \neq v_{2}^{\prime}$. Two cases are possible. First, if $u$ is not a descendant of $v$, then we add the edge $(u, v)$ to $T$ and delete the edge $\left(v_{1}, v_{2}\right)$ from $T$. Notice that the number of leaves increases, as two new vertices become leaves, $v_{1}$ and $v_{2}$, whereas in the worst case, at most one vertex stops being a leaf (vertex $u$ ). The second case is when $u$ is a descendant of $v$. Then we add an edge $(u, v)$ to $T$ and delete the edge $\left(v_{1}^{\prime}, v_{2}^{\prime}\right)$ from $T$. Again, the number of leaves increases by at least 1 , as both $v_{1}^{\prime}$ and $v_{2}^{\prime}$ are now leaves (see Figure 5 ).

We perform the preceding improvement step while possible. Let $T$ denote the final tree, where no such operation is possible. If $T$ has at least $L$ leaves, then we are done. Assume therefore that $T$ has fewer than $L$ leaves. Then the number of inner vertices of $T$ whose degree is greater than 2 in $T$ is at most $L$. If $P$ is a maximal 2-path in $T$, then the child of its lowermost vertex must be either a leaf or a vertex whose degree is more than 2 in $T$. Therefore, there are at most $2 L$ maximal 2-paths in $T$, and at least one such path must contain at least $\frac{n-2 L}{2 L} \geq p+4$ vertices. Let $P^{\prime}$ be the path obtained from $P$ by deleting the first two and the last two vertices. Then $P^{\prime}$ contains at least $p$ vertices, and since no improvement step was possible, $P^{\prime}$ must be a 2 -path in $Z$. 


\section{A.3. Proof of Lemma 2.19}

The proof that we provide here was suggested by Seymour (pers. comm.). A different proof, using stable matchings, was shown by Conforti et al. [2003].

Let $\hat{G}^{\prime} \subseteq \hat{G}$ be obtained from the union of the paths in $\mathcal{X}_{1} \cup \mathcal{X}_{2}$. Let $U_{1}^{\prime} \subseteq U_{1}$ be the set of vertices where the paths of $\mathcal{X}_{1}$ originate, and define $U_{2}^{\prime} \subseteq U_{2}$ for the set $\mathcal{X}_{2}$ of paths similarly. Let $E_{1}$ be the set of all edges participating in the paths in $\mathcal{X}_{1}$. While there is an edge $e \in E\left(\hat{G}^{\prime}\right) \backslash E_{1}$ such that graph $\hat{G}^{\prime} \backslash\{e\}$ contains a set of $\ell_{2}$ nearly disjoint $U_{2}^{\prime}-s$ paths, we delete $e$ from $\hat{G}^{\prime}$. At the end of this procedure, the final graph $\hat{G}^{\prime}$ has the property that for every edge $e \in E\left(\hat{G}^{\prime}\right) \backslash E_{1}$, the largest number of nearly disjoint $U_{2}^{\prime}-s$ paths in graph $\hat{G}^{\prime} \backslash\{e\}$ is less than $\ell_{2}$. Notice that $\mathcal{X}_{1} \subseteq \hat{G}^{\prime}$, and graph $\hat{G}^{\prime}$ contains $\ell_{2}$ nearly disjoint $U_{2}^{\prime}-s$ paths. We need the following claim.

ClaIm A.5. There is a set $\mathcal{X}^{\prime}$ of $\ell_{1}$ nearly disjoint $\left(U_{1}^{\prime} \cup U_{2}^{\prime}\right)-$ s paths in graph $\hat{G}^{\prime}$ such that exactly $\ell_{2}$ paths of $\mathcal{X}^{\prime}$ originate at the vertices of $U_{2}^{\prime}$.

Before we prove Claim A.5, we show that the set $\mathcal{X}^{\prime}$ of paths has the properties required by Lemma 2.19. Let $\mathcal{X}_{1}^{\prime} \subseteq \mathcal{X}^{\prime}$ be the set of paths originating from the vertices of $U_{1}^{\prime} \backslash U_{2}^{\prime}$, and let $\mathcal{X}_{2}^{\prime}=\mathcal{X}^{\prime} \backslash \mathcal{X}_{1}^{\prime}$. We only need to show that $\mathcal{X}_{1}^{\prime} \subseteq \mathcal{X}_{1}$. Assume otherwise. Then there is some edge $e \in E\left(\hat{G}^{\prime}\right) \backslash E_{1}$, which lies on some path in $\mathcal{X}_{1}^{\prime}$. But then $\mathcal{X}_{2}^{\prime} \subseteq \hat{G}^{\prime} \backslash\{e\}$, and edge $e$ should have been removed from graph $\hat{G}^{\prime}$. It now only remains to prove Claim A.5.

Proof of Claim A.5. The proof follows standard arguments. We construct a directed node-capacitated flow network $H$ : start from graph $\hat{G}^{\prime}$, and assign capacity 1 to each vertex of $\hat{G}^{\prime}$, except for vertex $s$, whose capacity is $\ell_{1}$. We add two new vertices: vertex $t_{1}$ of capacity $\ell_{1}-\ell_{2}$, which connects to every vertex of $U_{1}^{\prime}$ with a directed edge, and vertex $t_{2}$ of capacity $\ell_{2}$, which connects to every vertex of $U_{2}^{\prime}$ with a directed edge. Finally, we add a vertex $t$ of capacity $\ell_{1}$, which connects to $t_{1}$ and $t_{2}$ with directed edges. It is enough to show that there is a valid $t$-s flow of value $\ell_{1}$ in the resulting flow network: we can then use the integrality of flow to obtain an integral flow of the same value, which in turn immediately defines the desired set $\mathcal{X}^{\prime}$ of paths.

Assume for contradiction that there is no $t$-s flow of value $\ell_{1}$ in $H$. Then there is a set $Z$ of vertices, whose total capacity is less than $\ell_{1}$, such that $H \backslash Z$ contains no path connecting $t$ to $s$. Since the capacities of $t$ and $s$ are $\ell_{1}$ each, $s, t \notin Z$. In addition, since the capacities of $t_{1}$ and $t_{2}$ sum up to $\ell_{1}$, both of these vertices cannot simultaneously belong to $Z$.

Assume first that $t_{1} \in Z$. Then, since $t_{2} \notin Z$, set $Z$ contains at most $\ell_{2}-1$ additional vertices, each of which must have capacity 1 . Since there is a set of $\ell_{2}$ nearly disjoint $U_{2}^{\prime}-s$ paths in $\hat{G}^{\prime}$, at least one such path $P$ is disjoint from $Z$, and so $H \backslash Z$ must contain a path connecting $t$ to $s$, a contradiction.

Similarly, if $t_{2} \in Z$, then $t_{1} \notin Z$, and set $Z$ contains at most $\ell_{1}-\ell_{2}-1$ additional vertices, whose capacities must be all unit. But then at least one path in $\mathcal{X}_{1}$ is disjoint from $Z$, giving a path connecting $t$ to $s$ in $H \backslash Z$, a contradiction.

Therefore, we assume that all vertices of $Z$ are capacity- 1 vertices, which belong to $\hat{G}^{\prime}$. But then $Z$ contains at most $\ell_{1}-1$ vertices, so at least one path in $\mathcal{X}_{1}$ is disjoint from $Z$, giving again a path connecting $t$ to $s$ in $H \backslash Z$, a contradiction.

\section{A.4. Proof of Theorem 2.21}

Since $G$ has treewidth $k$, we can efficiently find a set $X$ of $\Omega(k)$ vertices of $G$ with properties guaranteed by Lemma 2.17 . 
Using the cut-matching game and Theorem 2.20, we can embed an expander $H=(X, F)$ into $G$ as follows. Each iteration $j$ of the cut-matching game requires the matching player to find a matching $M_{j}$ between a given partition of $X$ into two equal-size sets $Y_{j}, Z_{j}$. From Lemma 2.17, there exist a collection $\mathcal{P}_{j}$ of paths from $Y_{j}$ to $Z_{j}$, which cause congestion at most $1 / \alpha^{*}$ on the vertices of $G$; these paths naturally define the required matching $M_{j}$. The game terminates in $\gamma_{\mathrm{CMG}}(|X|)$ steps. Consider the collection of paths $\mathcal{P}=\bigcup_{j} \mathcal{P}_{j}$, and let $G^{\prime}$ be the subgraph of $G$ obtained by taking the union of these paths. Let $H=(X, F)$ be the expander, whose vertex set is $X$ and edge set is $F=\bigcup_{j} M_{j}$. By the construction, for each $j$, a node $v$ of $G$ appears in at most $1 / \alpha^{*}$ paths in $\mathcal{P}_{j}$. Therefore, the maximum vertex degree in $G^{\prime}$ is at most $2 \gamma_{\mathrm{CMG}}(|X|) / \alpha^{*}=O\left(\log ^{3} k\right)$, and moreover the vertex- (and hence also edge-) congestion caused by the set $\mathcal{P}$ of paths in $G$ is also upper bounded by the same quantity. We apply the algorithm $\mathcal{A}_{\mathrm{ARV}}$ to the sparsest cut instance defined by the graph $H$, where all vertices of $H$ serve as terminals. If the outcome is a cut whose sparsity is less than $\alpha_{\mathrm{CMG}}(|X|)$, then the algorithm fails; we discard the current graph $H$ and repeat the algorithm again. Otherwise, if the outcome is a cut of sparsity at least $\alpha_{\mathrm{CMG}}(|X|)$, then we are guaranteed that $X$ is an $\alpha_{\mathrm{CMG}}(|X|) / \beta_{\mathrm{ARV}}(|X|)=\Omega(\sqrt{\log |X|})$-expander, and in particular, it is an $\alpha$-expander, for $\alpha=\frac{1}{2}$. Since each execution of the cut-matching game is guaranteed to succeed with a constant probability, after $|X|$ such executions, the algorithm is guaranteed to succeed with high probability.

Since $H=(X, F)$ is an $\alpha$-expander, $X$ is $\alpha$-well-linked in $H$. Since $H$ is embedded into $G^{\prime}$ with congestion at most $2 \gamma_{\mathrm{CMG}}(|X|) / \alpha^{*}, X$ is $\frac{\alpha \cdot \alpha^{*}}{2 \gamma_{\mathrm{CMG}}(|X|)}=\Omega\left(\frac{1}{\log ^{3} k}\right)$-well-linked in $G^{\prime}$. Since the maximum vertex degree in $G^{\prime}$ is at most $2 \gamma_{\mathrm{CMG}}(|X|) / \alpha^{*}=O\left(\log ^{3} k\right)$, we can apply Theorem 2.14 to find a subset $X^{\prime} \subseteq X$ of $\Omega\left(\frac{k}{\log ^{15.5} k}\right)$ vertices such that $X^{\prime}$ is node-well-linked in $G^{\prime}$.

\section{B. PROOF OF THEOREM 3.2}

A set $\mathcal{L}$ of $w$ disjoint paths in $G$ that connect the vertices of $A$ to the vertices of $B$ is called an $A$ - $B$ linkage. Since the sets $A, B$ of vertices are linked in $G$, such a linkage $\mathcal{L}$ exists and can be found efficiently.

Given an $A$ - $B$ linkage $\mathcal{L}$, we construct a graph $H=H(\mathcal{L})$ as follows. The vertices of $H$ are $U=\left\{u_{P} \mid P \in \mathcal{L}\right\}$, and there is an edge between $u_{P}$ and $u_{P^{\prime}}$ if and only if there is a path $\gamma_{P, P^{\prime}}$ in $G$, whose first vertex belongs to $P$, last vertex belongs to $P^{\prime}$, and the inner vertices do not belong to any paths in $\mathcal{L}$. Notice that since $G$ is a connected graph, so is $H(\mathcal{L})$ for any $A-B$ linkage $\mathcal{L}$. We say that an $A-B$ linkage $\mathcal{L}$ is good if and only if the longest 2-path in the corresponding graph $H(\mathcal{L})$ contains fewer than $8 h_{1}+1$ vertices.

Assume first that we are given a good linkage $\mathcal{L}$ in $G$. Then Theorem 2.18 guarantees that there is a spanning tree $\tau$ in $H_{\mathcal{L}}$ with at least $\frac{w}{2\left(8 h_{1}+5\right)} \geq h_{2}$ leaves. We let $\mathcal{P}$ contain all paths $P \in \mathcal{L}$ whose corresponding vertex $u_{P}$ is a leaf of $\tau$. Then $\mathcal{P}$ contains at least $h_{2}$ node-disjoint paths, connecting vertices of $A$ to vertices of $B$. Consider any pair $P, P^{\prime} \in \mathcal{P}$ of paths with $P \neq P^{\prime}$, and let $Q$ be the path connecting $u_{P}$ to $u_{P^{\prime}}$ in $\tau$. Let $H_{P, P^{\prime}} \subseteq G$ be the graph consisting of the union of all paths $P^{\prime \prime}$ with $u_{P^{\prime \prime}} \in V(Q)$, and paths $\gamma_{P_{1}, P_{2}}$ where $\left(u_{P_{1}}, u_{P_{2}}\right)$ is an edge of $Q$. Then graph $H_{P, P^{\prime}}$ contains a path $\beta_{P, P^{\prime}}$, connecting a vertex of $P$ to a vertex of $P^{\prime}$, such that all inner vertices of $\beta_{P, P^{\prime}}$ are disjoint from $\bigcup_{P^{\prime \prime} \in \mathcal{P}} V\left(P^{\prime \prime}\right)$.

To complete the proof of Theorem 3.2, we show, using the following theorem, that we can either find a model of the $\left(h_{1} \times h_{1}\right)$-grid minor in $G$ or compute a good $A$ - $B$ linkage $\mathcal{L}$. 
Theorem B.1. There is an efficient algorithm that, given an A-B linkage $\mathcal{L}$, such that $\mathcal{L}$ is not a good linkage, returns one of the following:

- either a model of the $\left(h_{1} \times h_{1}\right)$-grid minor in $G$ or

- a new $A$-B linkage $\mathcal{L}^{\prime}$ such that the number of the degree-2 vertices in $H\left(\mathcal{L}^{\prime}\right)$ is strictly smaller than the number of the degree-2 vertices in $H(\mathcal{L})$.

The proof of Theorem 3.2 immediately follows from Theorem B.1. We start with an arbitrary $A-B$ linkage $\mathcal{L}$ and iterate. While $\mathcal{L}$ is not a good linkage, we apply Theorem B.1 to it. If the outcome is a model of the $\left(h_{1} \times h_{1}\right)$-grid minor, then we terminate the algorithm and return this model. Otherwise, if $\mathcal{L}^{\prime}$ is a good linkage, then we compute a subset $\mathcal{P} \subseteq \mathcal{L}^{\prime}$ of paths as described earlier and terminate the algorithm. Otherwise, we replace $\mathcal{L}$ with $\mathcal{L}^{\prime}$ and continue to the next iteration. After $O(w)$ iterations, the number of degree-2 vertices in the graph $H(\mathcal{L})$ is guaranteed to fall below $8 h_{1}+1$ (unless the algorithm terminates earlier). It now remains to prove Theorem B.1.

\section{B.1. Proof of Theorem B.1}

Since $\mathcal{L}$ is not a good $A-B$ linkage, there is a 2 -path $R^{*}=\left(u_{P_{0}}, \ldots, u_{P_{8 h_{1}}}\right)$ of length $8 h_{1}+1$ in the corresponding graph $H=H_{\mathcal{L}}$. Let $z=2 h_{1}$, and consider the following four subsets of paths, $\mathcal{P}_{1}=\left\{P_{1}, \ldots, P_{z}\right\}, \mathcal{P}_{2}=\left\{P_{z+1}, \ldots, P_{2 z}\right\}, \mathcal{P}_{3}=\left\{P_{2 z+1}, \ldots, P_{3 z}\right\}$, and $\mathcal{P}_{4}=\left\{P_{3 z+1}, \ldots, P_{4 z}\right\}$, whose corresponding vertices participate in the 2-path $R^{*}$. (Notice that $P_{0} \notin \mathcal{P}_{1}$, but the degree of $u_{P_{0}}$ is 2 in $H(\mathcal{L})$-we use this fact later.) Let $X \subseteq A$ be the set of the endpoints of the paths in $\mathcal{P}_{2}$ that belong to $A$, and let $Y \subseteq B$ be the set of the endpoints of the paths in $\mathcal{P}_{4}$ that belong to $B$ (Figure 6). Since $A, B$ are linked in $G$, we can find a set $\mathcal{Q}$ of $z$ disjoint paths connecting $X$ to $Y$ in $G$. We view the paths in $\mathcal{Q}$ as directed from $X$ to $Y$.

Let $Q \in \mathcal{Q}$ be any such path. Observe that since $R^{*}$ is a 2-path in $H(\mathcal{L})$, path $Q$ has to either intersect all paths in $\mathcal{P}_{1}$ or all paths in $\mathcal{P}_{3}$ before it reaches $Y$. Therefore, it must intersect $P_{z+1}$ or $P_{2 z}$. Let $v$ be the last vertex of $Q$ that belongs to $P_{z+1} \cup P_{2 z}$. Let $Q^{\prime}$ be the segment of $Q$ starting from $v$ and terminating at a vertex of $Y$. Assume first that $v \in P_{z+1}$. We say that $Q$ is a type-1 path in this case. Let $u$ be the first vertex on $Q^{\prime}$ that belongs to $P_{0}$. (Such a vertex must exist again because $R^{*}$ is a 2-path.) Let $Q^{*}$ be the segment of $Q^{\prime}$ between $v$ and $u$. Then $Q^{*}$ intersects every path in $\mathcal{P}_{1} \cup\left\{P_{0}, P_{z+1}\right\}$ and does not intersect any other path in $\mathcal{L}$, whereas $\left|V\left(Q^{*}\right) \cap V\left(P_{0}\right)\right|=\left|V\left(Q^{*}\right) \cap P_{z+1}\right|=1$ (see Figure 6).

Similarly, if $v \in P_{2 z}$, then we say that $Q$ is a type-2 path. Let $u$ be the first vertex of $Q^{\prime}$ that belongs to $P_{3 z+1}$, and let $Q^{*}$ be the segment of $Q^{\prime}$ between $u$ and $v$. Then $Q^{*}$ intersects every path in $\mathcal{P}_{3} \cup\left\{P_{2 z} \cup P_{3 z+1}\right\}$ and does not intersect any other path in $\mathcal{L}$, whereas $\left|V\left(Q^{*}\right) \cap V\left(P_{2 z}\right)\right|=\left|V\left(Q^{*}\right) \cap V\left(P_{3 z+1}\right)\right|=1$.

Clearly, either at least half the paths in $\mathcal{Q}$ are type-1 paths or at least half the paths in $\mathcal{Q}$ are type-2 paths. We assume without loss of generality that the former is true. Let $\mathcal{Q}^{\prime}$ be the set of the subpaths $Q^{*}$ for all type-1 paths $Q \in \mathcal{Q}$-that is, $\mathcal{Q}^{\prime}=\left\{Q^{*} \mid Q \in \mathcal{Q}\right.$ and $Q$ is type-1 $\}$. Then $\left|\mathcal{Q}^{\prime}\right| \geq z / 2=h_{1}$.

The rest of the proof is based on the following idea. We will show that either the graph obtained from the union of the paths in $\mathcal{Q}^{\prime} \cup \mathcal{P}_{1}$ is a planar graph, in which case we recover a grid minor directly, or we will find a new $A$ - $B$ linkage $\mathcal{L}^{\prime}$, such that $H\left(\mathcal{L}^{\prime}\right)$ contains fewer degree-2 vertices than $H(\mathcal{L})$. To accomplish this, we will iteratively simplify the intersection pattern of the paths in $\mathcal{Q}^{\prime}$ and $\mathcal{P}_{1}^{\prime}$.

The algorithm performs several iterations. Throughout the algorithm, the set $\mathcal{Q}^{\prime}$ of paths remains unchanged. The input to every iteration consists of a set $\mathcal{P}_{1}^{\prime}$ of paths such that the following hold: 


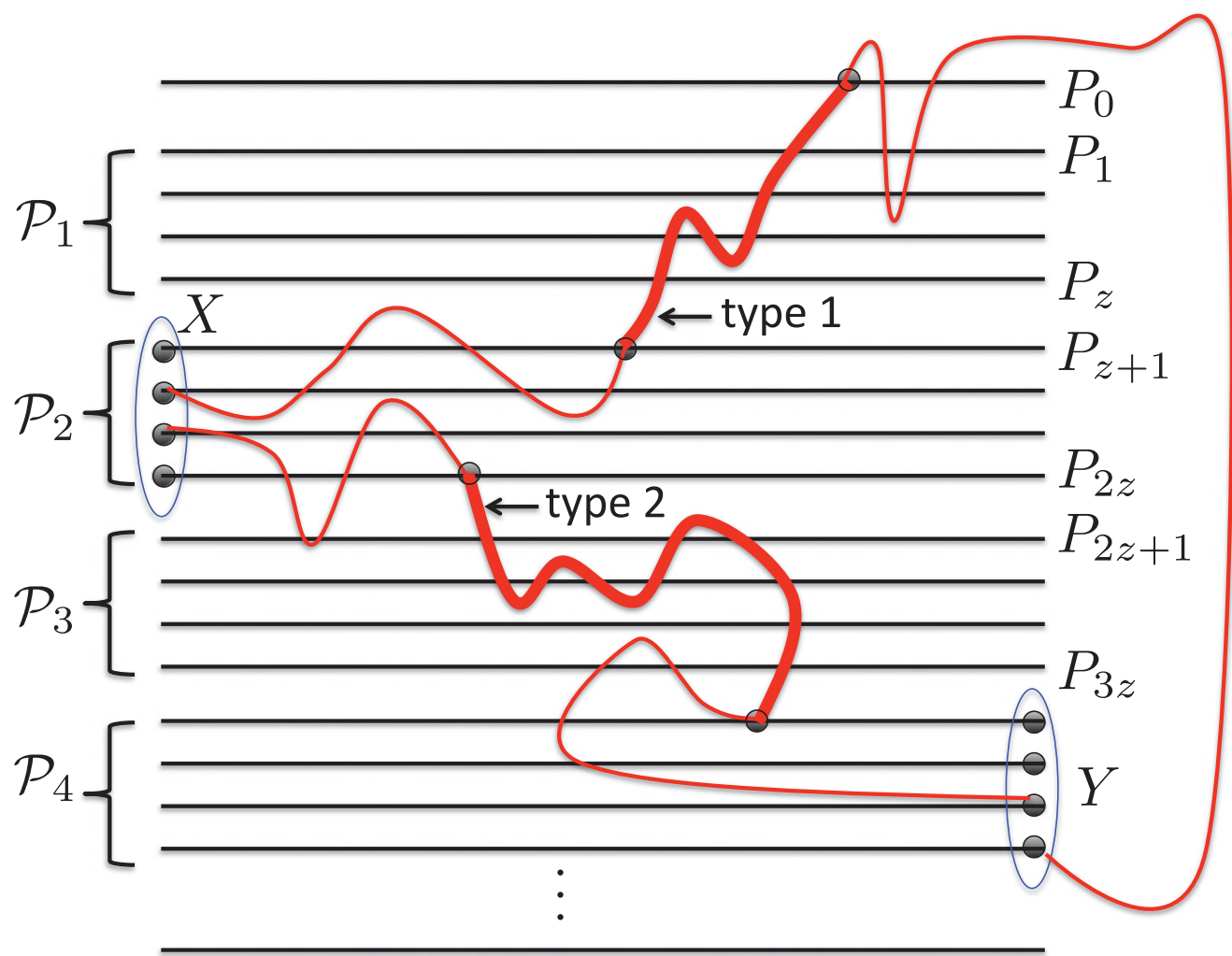

Fig. 6. Two examples for paths in $\mathcal{Q}$ - a type- 1 and a type-2 path-are shown in red, with the $Q^{*}$ segment highlighted.

$-\mathcal{L}^{\prime}=\left(\mathcal{L} \backslash \mathcal{P}_{1}\right) \cup \mathcal{P}_{1}^{\prime}$ is an $A-B$ linkage;

- the graphs $H=H(\mathcal{L})$ and $H^{\prime}=H\left(\mathcal{L}^{\prime}\right)$ are isomorphic to each other, where the vertices $u_{P}$ for $P \notin \mathcal{P}_{1}$ are mapped to themselves; and

- every path in $\mathcal{Q}^{\prime}$ intersects every path in $\mathcal{P}_{1}^{\prime} \cup\left\{P_{0}, P_{z+1}\right\}$ and no other paths of $\mathcal{L}^{\prime}$.

The input to the first iteration is $\mathcal{P}_{1}^{\prime}=\mathcal{P}_{1}$. Throughout the algorithm, we maintain a graph $\tilde{H}$ - the subgraph of $G$ induced by the edges participating in the paths of $\mathcal{P}_{1}^{\prime} \cup \mathcal{Q}^{\prime}$. We define below two combinatorial objects: a bump and a cross. We show that if $\tilde{H}$ has either a bump or a cross, then we can find a new set $\mathcal{P}_{1}^{\prime \prime}$ of paths such that $\mathcal{L}^{\prime \prime}=\left(\mathcal{L}^{\prime} \backslash \mathcal{P}_{1}^{\prime}\right) \cup \mathcal{P}_{1}^{\prime \prime}$ is an $A$ - $B$ linkage. Moreover, either $H^{\prime \prime}=H\left(\mathcal{L}^{\prime \prime}\right)$ contains fewer degree-2 vertices than $H^{\prime}$ or the two graphs are isomorphic to each other. In the former case, we terminate the algorithm and return the linkage $\mathcal{L}^{\prime \prime}$. In the latter case, we show that we obtain a valid input to the next iteration, and $\left|E\left(\mathcal{Q}^{\prime}\right) \cup E\left(\mathcal{P}_{1}^{\prime}\right)\right|>\left|E\left(\mathcal{Q}^{\prime}\right) \cup E\left(\mathcal{P}_{1}^{\prime \prime}\right)\right|$. In other words, the number of edges in the graph $\tilde{H}$ strictly decreases in every iteration. We also show that if $\tilde{H}$ contains no bump and no cross, then a large subgraph of $\tilde{H}$ is planar and contains a grid minor of size $\left(h_{1} \times h_{1}\right)$. Therefore, after $|E(G)|$ iterations, the algorithm is guaranteed to terminate with the desired output. We now proceed to define the bump and the cross, as well as their corresponding actions. We recall the useful observation that for any $A-B$ linkage $\mathcal{L}^{\prime}$, the corresponding graph $H\left(\mathcal{L}^{\prime}\right)$ is a connected graph since $G$ is connected. 

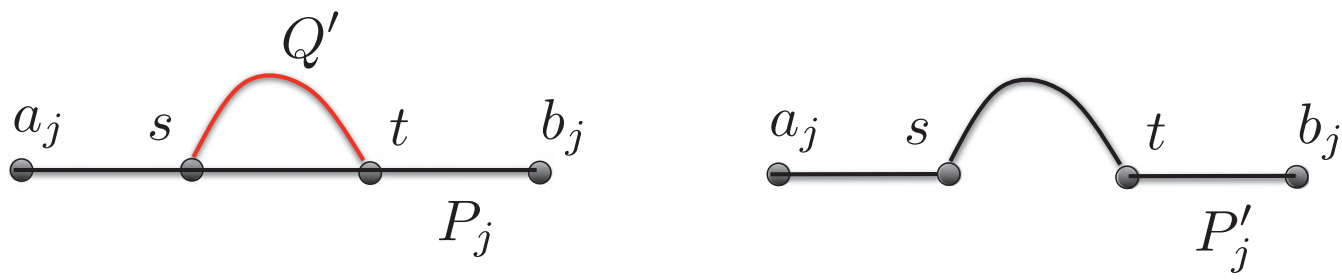

Fig. 7. A bump and the corresponding action.

A bump. Let $\mathcal{P}_{1}^{\prime}$ be the current set of paths, and let $\mathcal{L}^{\prime}=\left(\mathcal{L} \backslash \mathcal{P}_{1}\right) \cup \mathcal{P}_{1}^{\prime}$ be the corresponding linkage. We say that the corresponding graph $\tilde{H}$ contains a bump if there is a subpath $Q^{\prime}$ of some path $Q \in \mathcal{Q}^{\prime}$ whose endpoints, $s$ and $t$, both belong belong to the same path $P_{j} \in \mathcal{P}_{1}^{\prime}$, and all inner vertices of $Q^{\prime}$ are disjoint from all paths in $\mathcal{P}_{1}^{\prime}$. (Figure 7). Let $a_{j} \in A, b_{j} \in B$ be the endpoints of $P_{j}$, and assume that $s$ appears before $t$ on $P_{j}$, as we traverse it from $a_{j}$ to $b_{j}$. Let $P_{j}^{\prime}$ be the path obtained from $P_{j}$ by concatenating the segment of $P_{j}$ between $a_{j}$ and $s$, the path $Q^{\prime}$, and the segment of $P_{j}$ between $t$ and $b_{j}$.

Let $\mathcal{P}_{1}^{\prime \prime}$ be the set of paths obtained by replacing $P_{j}$ with $P_{j}^{\prime}$ in $\mathcal{P}_{1}^{\prime}$, and let $\mathcal{L}^{\prime \prime}=$ $\left(\mathcal{L}^{\prime} \backslash \mathcal{P}_{1}^{\prime}\right) \cup \mathcal{P}_{1}^{\prime \prime}=\left(\mathcal{L} \backslash \mathcal{P}_{1}\right) \cup \mathcal{P}_{1}^{\prime \prime}$. It is immediate to verify that $\mathcal{L}^{\prime \prime}$ is an $A$ - $B$ linkage. Let $H^{\prime}=H\left(\mathcal{L}^{\prime}\right)$ and $H^{\prime \prime}=H\left(\mathcal{L}^{\prime \prime}\right)$, and let $E^{\prime}$ be the set of edges in the symmetric difference of the two graphs (i.e., edges that belong to exactly one of the two graphs). Then for every edge in $E^{\prime}$, both endpoints must belong to the set $\left\{u_{P_{j-1}}, u_{P_{j}}, u_{P_{j+1}}\right\}$; this is because the vertices $\left\{u_{P} \mid P \in \mathcal{P}_{1}\right\}$ are part of a 2-path in $H(\mathcal{L})$. In particular, the only vertices whose degree may be different in the two graphs are $u_{P_{j-1}}, u_{P_{j}}, u_{P_{j+1}}$. If the degree of any one of these three vertices is different in $H^{\prime \prime}$ and $H^{\prime}$, then, since their degrees are 2 in both $H^{\prime}$ and the original graph $H$, we obtain a new $A$ - $B$ linkage $\mathcal{L}^{\prime \prime}$ such that $H\left(\mathcal{L}^{\prime \prime}\right)$ contains fewer degree- 2 vertices than $H$. Otherwise, if the degrees of all three vertices remain equal to 2 , then it is immediate to verify that $H^{\prime \prime}$ is isomorphic to $H^{\prime}$, where each vertex is mapped to itself, except we replace $u_{P_{j}}$ with $u_{P_{j^{\prime}}}$. It is easy to verify that all invariants continue to hold in this case. Let $\tilde{H}$ be the graph obtained by the union of the paths in $\mathcal{P}_{1}^{\prime}$ and $\mathcal{Q}^{\prime}$, and define $\tilde{H}^{\prime}$ similarly for $\mathcal{P}_{1}^{\prime \prime}$ and $\mathcal{Q}^{\prime}$. Then $\tilde{H}^{\prime}$ contains fewer edges than $\tilde{H}$, as the portion of the path $P_{j}$ between $s$ and $t$ belongs to $\tilde{H}$ but not to $\tilde{H}^{\prime}$.

A cross. Suppose that we are given two disjoint paths $Q_{1}^{\prime}, Q_{2}^{\prime}$, where $Q_{1}^{\prime}$ is a subpath of some path $Q_{1} \in \mathcal{Q}^{\prime}$, and $Q_{2}^{\prime}$ is a subpath of some path $Q_{2} \in \mathcal{Q}^{\prime}$ (with possibly $Q_{1}=Q_{2}$ ). Assume that the endpoints of $Q_{1}^{\prime}$ are $s_{1}, t_{1}$ and that the endpoints of $Q_{2}^{\prime}$ are $s_{2}, t_{2}$. Moreover, suppose that $s_{1}, s_{2}$ appear on some path $P_{j} \in \mathcal{P}_{1}^{\prime}$ in this order and that $t_{2}, t_{1}$ appear on $P_{j+1} \in \mathcal{P}_{1}^{\prime}$ in this order (where the paths in $\mathcal{P}_{1}^{\prime}$ are directed from $A$ to $B$ ), and no inner vertex of $Q_{1}^{\prime}$ or $Q_{2}^{\prime}$ belongs to any path in $\mathcal{P}_{1}^{\prime}$. We then say that $Q_{1}^{\prime}, Q_{2}^{\prime}$ are a cross (Figure 8).

Given a cross as defined previously, we define two new paths as follows. Assume that the endpoints of $P_{j}$ are $a_{j} \in A, b_{j} \in B$, and similarly the endpoints of $P_{j+1}$ are $a_{j+1} \in A$, $b_{j+1} \in B$. Let $P_{j}^{\prime}$ be obtained by concatenating the segment of $P_{j}$ between $a_{j}$ and $s_{1}$, the path $Q_{1}^{\prime}$, and the segment of $P_{j+1}$ between $t_{1}$ and $b_{j+1}$. Let $P_{j+1}^{\prime}$ be obtained by concatenating the segment of $P_{j+1}$ between $a_{j+1}$ and $t_{2}$, the path $Q_{2}^{\prime}$, and the segment of $P_{j}$ between $s_{2}$ and $b_{j}$. We obtain the new set $\mathcal{P}_{1}^{\prime \prime}$ of paths by replacing $P_{j}, P_{j+1}$ with $P_{j}^{\prime}, P_{j+1}^{\prime}$ in $\mathcal{P}_{1}^{\prime}$. Let $\mathcal{L}^{\prime \prime}=\left(\mathcal{L}^{\prime} \backslash \mathcal{P}_{1}^{\prime}\right) \cup \mathcal{P}_{1}^{\prime \prime}=\left(\mathcal{L} \backslash \mathcal{P}_{1}\right) \cup \mathcal{P}_{1}^{\prime \prime}$. It is immediate to verify that $\mathcal{L}^{\prime \prime}$ is an $A$-B linkage. As before, let $H^{\prime}=H\left(\mathcal{L}^{\prime}\right)$ and $H^{\prime \prime}=H\left(\mathcal{L}^{\prime \prime}\right)$, and let $E^{\prime}$ be the set of edges in the symmetric difference of the two graphs. Then for every edge in $E^{\prime}$, both endpoints must belong to the set $\left\{u_{P_{j-1}}, u_{P_{j}}, u_{P_{j+1}}, u_{P_{j+2}}\right\}$. Again, this is because the 

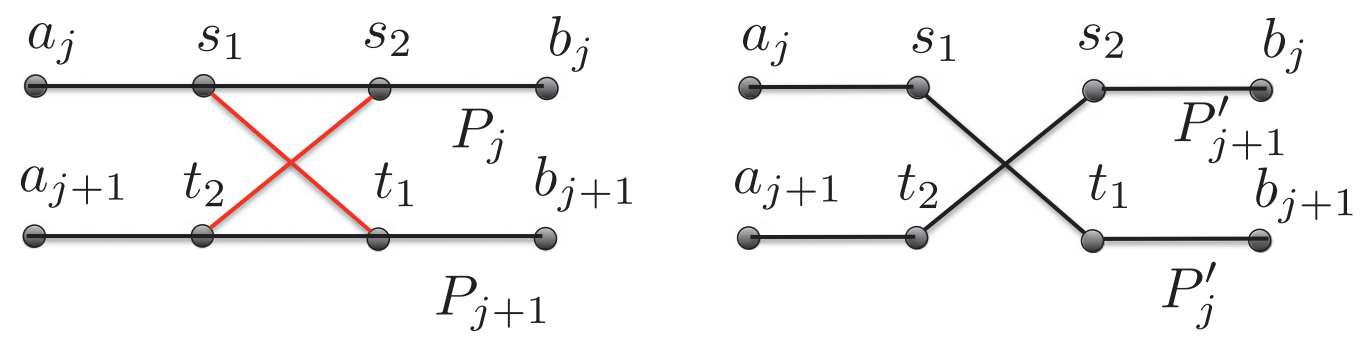

Fig. 8. A cross and the corresponding action.

vertices $\left\{u_{P} \mid P \in \mathcal{P}_{1}\right\}$ are part of a 2 -path in $H(\mathcal{L})$. The only vertices whose degree may be different in the two graphs are $u_{P_{j-1}}, u_{P_{j}}, u_{P_{j+1}}, u_{P_{j+2}}$. If the degree of any one of these four vertices is different in $H^{\prime \prime}$ and $H^{\prime}$, then, since their degrees are 2 in both $H^{\prime}$ and the original graph $H$, we obtain a new $A-B$ linkage $\mathcal{L}^{\prime \prime}$ such that $H\left(\mathcal{L}^{\prime \prime}\right)$ contains fewer degree-2 vertices than $H$. Otherwise, if the degrees of all four vertices remain equal to 2 , then it is immediate to verify that $H^{\prime \prime}$ is isomorphic to $H^{\prime}$, where each vertex is mapped to itself, except we replace $u_{P_{j}}, u_{P_{j+1}}$ with $u_{P_{j}^{\prime}}, u_{P_{j+1}^{\prime}}$ (possibly switching them). It is easy to verify that all invariants continue to hold in this case. Let $\tilde{H}$ be the graph obtained by the union of the paths in $\mathcal{P}_{1}^{\prime} \cup \mathcal{Q}^{\prime}$, and define $\tilde{H}^{\prime}$ similarly for $\mathcal{P}_{1}^{\prime \prime} \cup \mathcal{Q}^{\prime}$. Then $\tilde{H}^{\prime}$ contains fewer edges than $\tilde{H}$, as the portion of the path $P_{j}$ between $s_{1}$ and $s_{2}$ belongs to $\tilde{H}$ but not to $\tilde{H}^{\prime}$.

We are now ready to complete the description of our algorithm. We start with $\mathcal{P}_{1}^{\prime}=\mathcal{P}_{1}$ and then iterate. In every iteration, we construct a graph $\tilde{H}$ - the subgraph of $G$ induced by $\mathcal{P}_{1}^{\prime} \cup \mathcal{Q}^{\prime}$. If $\tilde{H}$ contains a bump or a cross, we apply the appropriate action. If the resulting linkage $\mathcal{L}^{\prime \prime}$ has the property that $H\left(\mathcal{L}^{\prime \prime}\right)$ has fewer degree-2 vertices than $H(\mathcal{L})$, then we terminate the algorithm and return $\mathcal{L}^{\prime \prime}$. Otherwise, we obtain a valid input to the next iteration, and moreover, the number of edges in the new graph $\tilde{H}$ strictly decreases. Therefore, we are guaranteed that within $O(|E(G)|)$ iterations, either the algorithm terminates with the desired linkage $\mathcal{L}^{\prime \prime}$ or the graph $\tilde{H}$ contains no bump and no cross. We now assume that the latter happens.

Consider the final graph $\tilde{H}$. For each path $Q \in \mathcal{Q}^{\prime}$, let $v_{Q}$ be the first vertex of $Q$ that belongs to $V\left(\mathcal{P}_{1}^{\prime}\right)$, and let $u_{Q}$ be the last vertex of $Q$ that belongs to $V\left(\mathcal{P}_{1}^{\prime}\right)$. Let $\tilde{Q}$ be the subpath of $Q$ between $v_{Q}$ and $u_{Q}$. Delete from $\tilde{H}$ all vertices of $V(Q) \backslash V(\tilde{Q})$ for all $Q \in \mathcal{Q}^{\prime}$, and let $\tilde{H}^{\prime}$ denote the resulting graph. Let $\tilde{\mathcal{Q}}=\{\tilde{Q} \mid Q \in \mathcal{Q}\}$. We need the following claim.

\section{Claim B.2. If $\tilde{H}$ contains no cross and no bump, then $\tilde{H}^{\prime}$ is planar.}

Proof. Consider some path $\tilde{Q} \in \tilde{\mathcal{Q}}$. Delete from $\tilde{Q}$ all edges that participate in the paths in $\mathcal{P}_{1}^{\prime}$, and let $\Sigma(\tilde{Q})$ be the resulting set of subpaths of $\tilde{Q}$. While some path $\sigma \in \Sigma(\tilde{Q})$ contains a vertex $v \in V\left(\mathcal{P}_{1}^{\prime}\right)$ as an inner vertex, we replace $\sigma$ with two subpaths, where each subpath starts at one of the endpoints of $\sigma$ and terminates at $v$. Let $\Sigma=\bigcup_{\tilde{Q} \in \tilde{\mathcal{Q}}} \Sigma(\tilde{Q})$ be the resulting set of paths. Then for each path $\sigma \in \Sigma$, both endpoints of $\sigma$ belong to $V\left(\mathcal{P}_{1}^{\prime}\right)$, and the inner vertices are disjoint from $V\left(\mathcal{P}_{1}^{\prime}\right)$. Moreover, since the paths in $\mathcal{P}_{1}^{\prime}$ induce a 2-path in the corresponding graph $H\left(\mathcal{L}^{\prime}\right)$, and since there are no bumps, the endpoints of each such path $\sigma$ connect two consecutive paths in $\mathcal{P}_{1}^{\prime}$. Since no crosses are allowed, it is easy to see that the graph $\tilde{H}^{\prime}$ is planar. 
We now show how to construct a grid minor in graph $\tilde{H}^{\prime}$. We start from the union of the paths in $\mathcal{P}_{1}^{\prime}$ and $\tilde{\mathcal{Q}}$, and perform the following transformation. We say that a segment $\sigma$ of a path $Q \in \tilde{\mathcal{Q}}$ is a hill if and only if (i) the endpoints $s, t$ of $\sigma$ lie on some path $P_{i} \in \mathcal{P}_{1}^{\prime}$; (ii) the segment $\sigma^{\prime}$ of $P_{i}$ whose endpoints are $s$ and $t$ does not contain any vertex of $V(\tilde{\mathcal{Q}} \backslash\{Q\})$; and (iii) $\sigma$ intersects $P_{i-1}$ and is internally disjoint from all vertices of $V\left(\mathcal{P}_{1}^{\prime} \backslash\left\{P_{i-1}\right\}\right)$. While there is a hill in $\mathcal{P}_{1}^{\prime} \cup \tilde{\mathcal{Q}}$, we modify the corresponding path $Q$ by replacing the segment $\sigma$ with $\sigma^{\prime}$. If this creates a cycle on $Q$ (this can happen if $\sigma^{\prime}$ contained a vertex of $Q$ ), we discard all such cycles until $Q$ becomes a simple path. We continue performing such transformations until there is no hill in the set $\mathcal{P}_{1}^{\prime} \cup \tilde{\mathcal{Q}}$ of paths. Notice that this transformation cannot create any bumps. We need the following claim.

Claim B.3. When the preceding algorithm terminates, for all $P_{i} \in \mathcal{P}_{1}^{\prime}$ and $Q \in \tilde{\mathcal{Q}}$, $P_{i} \cap Q$ is a path.

Notice that it is now immediate to obtain the $\left(h_{1} \times h_{1}\right)$-grid minor from the union of the paths in $\mathcal{P}_{1}^{\prime} \cup \tilde{\mathcal{Q}}$ by first contracting every path $P_{i} \cap Q$ for all $P_{i} \in \mathcal{P}_{1}^{\prime}$ and $Q \in \tilde{\mathcal{Q}}$, and then suppressing all degree-2 vertices, after which we discard the $h_{1}$ extra rows. It is now enough to prove Claim B.3.

Proof. Assume otherwise. Then there must be some path $Q \in \mathcal{Q}$, and some segment $\sigma$ of $Q$, whose two endpoints $s, t$ lie on some path $P_{i} \in \mathcal{P}_{1}^{\prime}$, such that $\sigma$ intersects $P_{i-1}$, and it is internally disjoint from $V\left(\mathcal{P}_{1}^{\prime} \backslash\left\{P_{i}\right\}\right)$. Notice that since there are no bumps, the intersection of $Q$ and $P_{i-1}$ is a path. Among all such pairs $\left(Q, P_{i}\right)$, choose the one maximizing $i$. Since $\sigma$ is not a hill, there must be some path $Q^{\prime} \neq Q$ in $\tilde{\mathcal{Q}}$ that intersects the segment $\sigma^{\prime}$ of $P_{i}$, lying between $s$ and $t$. Let $v$ be any vertex in $Q^{\prime} \cap \sigma^{\prime}$, and let $\sigma^{\prime \prime}$ be the longest contiguous subpath of $Q^{\prime}$ contained in $\sigma^{\prime}$. Let $u$ be the last vertex of $Q^{\prime}$ before $\sigma^{\prime \prime}$ that belongs to $V\left(\mathcal{P}_{1}^{\prime}\right)$, and let $u^{\prime}$ be the first vertex of $Q^{\prime}$ after $\sigma^{\prime \prime}$ that belongs to $V\left(\mathcal{P}_{1}^{\prime}\right)$. Then it is easy to verify that $u, u^{\prime} \in V\left(P_{i+1}\right)$ (and in particular $i \neq 2 h_{1}$ ). But then we should have chosen the pair $\left(Q^{\prime}, P_{i+1}\right)$ instead of $\left(Q, P_{i}\right)$, a contradiction.

\section{PROOF OF COROLLARY 3.3}

We say that a cluster $S_{i} \in \mathcal{S}$ is even if $i$ is even, and otherwise we say that $S_{i}$ is odd. We apply Theorem 3.2 to graph $G\left[S_{i}\right]$ for every even cluster $S_{i}$ using $A=A_{i}$ and $B=B_{i}$. If, for any even cluster $S_{i}$, the outcome is the $\left(h_{1} \times h_{1}\right)$-grid minor, then we terminate the algorithm and return the model of this minor. Therefore, we assume that for every even index $i$, Theorem 3.2 returns a collection $\mathcal{L}_{i}$ of $h_{2}$ node-disjoint paths contained in $G\left[S_{i}\right]$, which connect some subset $A_{i}^{\prime} \subseteq A_{i}$ of $h_{2}$ vertices to a subset $B_{i}^{\prime} \subseteq B_{i}$ of $h_{2}$ vertices, such that for every pair $P, P^{\prime} \in \mathcal{L}_{i}$ of paths, there is a path $\beta_{i}\left(P, P^{\prime}\right)$ in $G\left[S_{i}\right]$, connecting a vertex of $P$ to a vertex of $P^{\prime}$, where $\beta_{i}\left(P, P^{\prime}\right)$ is internally disjoint from $V\left(\mathcal{L}_{i}\right)$.

Fix some $1 \leq i \leq\lfloor\ell / 2\rfloor$. Let $A_{2 i}^{\prime} \subseteq A_{2 i}$ and $B_{2 i}^{\prime} \subseteq B_{2 i}$ be the sets of endpoints of the paths in $\mathcal{L}_{2 i}$. Let $\mathcal{L}_{2 i-1}^{-} \subseteq \mathcal{P}_{2 i-1}$ be the set of paths terminating at the vertices of $A_{2 i}^{\prime}$. If $2 i<\ell$, then let $\mathcal{L}_{2 i}^{+} \subseteq \mathcal{P}_{2 i}$ be the set of paths originating at the vertices of $B_{2 i}^{\prime}$; otherwise, let $\mathcal{L}_{2 i}^{+}$contain $h_{2}$ paths, each of which consists of a single distinct vertex of $B_{2 i}^{\prime}$.

We use the odd clusters, via the well-linkedness properties of the clusters, to connect the path collections from the even clusters into the desired path collection $\mathcal{Q}$.

Consider now some odd-indexed cluster $S_{i}$. If $i \neq 1$, then let $A_{i}^{\prime} \subseteq A_{i}$ be the set of vertices where the paths of $\mathcal{L}_{i-1}^{+}$terminate, and otherwise let $A_{i}^{\prime}$ be any set of $h_{2}$ vertices of $A_{i}$. If $i<\ell$, then let $B_{i}^{\prime} \subseteq B_{i}$ be the set of vertices where the paths of $\mathcal{L}_{i+1}^{-}$originate, and otherwise let $B_{i}^{\prime}$ be any set of $h_{2}$ vertices of $B_{i}$. Since $A_{i}, B_{i}$ are linked in $G\left[S_{i}\right]$, 
there is a set $\mathcal{R}_{i}$ of $h_{2}$ node-disjoint paths that are contained in $G\left[S_{i}\right]$ and connect $A_{i}^{\prime}$ to $B_{i}^{\prime}$.

We now define a the set $\mathcal{Q}$ of paths, obtained by the concatenation of all paths $\mathcal{L}_{i}^{-}, \mathcal{L}_{i}, \mathcal{L}_{i}^{+}$where $S_{i}$ is an even cluster, and paths $\mathcal{R}_{j}$, where $S_{j}$ is an odd cluster. The resulting set $\mathcal{Q}$ contains $h_{2}$ disjoint paths, originating at the vertices of $A_{1}$ and terminating at the vertices of $B_{\ell}$, where for every $1 \leq i \leq \ell$, for every path $Q \in \mathcal{Q}$, $Q \cap S_{i}$ is a path, and $S_{1} \cap Q, S_{2} \cap Q, \ldots, S_{\ell} \cap Q$ appear on $Q$ in this order. Moreover, for every even integer $1 \leq i \leq \ell$, for every pair $Q, Q^{\prime} \in \mathcal{Q}$ of paths, there is a path $\beta_{i}\left(Q, Q^{\prime}\right) \subseteq G\left[S_{i}\right]$ that connects a vertex of $Q$ to a vertex of $Q^{\prime}$ and is internally disjoint from all paths in $\mathcal{Q}$. It is immediate to verify that all paths in $\mathcal{Q}$ are contained in $G^{\prime}$.

\section{C.1. Proof of Corollary 3.4}

We apply Corollary 3.3 to the path-of-sets system, with parameters $h_{1}=g$ and $h_{2}=g$, so $w \geq 16 g^{2}+10 g$ as required. If the outcome is the $(g \times g)$-grid minor, then we terminate the algorithm and return its model. Therefore, we assume that the outcome of Corollary 3.3 is a set $\mathcal{Q}$ of $g$ paths connecting vertices of $A_{1}$ to vertices of $B_{\ell}$, which we denote by $\mathcal{Q}=\left\{Q_{1}, \ldots, Q_{g}\right\}$ (the ordering is arbitrary).

Consider the following graph $G^{*}$. Start with a grid containing $g$ rows and $g(g-1)$ columns, with the columns indexed $C_{0}, C_{1}, \ldots, C_{g(g-1)-1}$ from left to right. For all $0 \leq$ $i<g(g-1)$, let $t_{i}=i \bmod (g-1)$. We delete from the $i$ th column all edges except for the $\left(t_{i}+1\right)$ th edge from the top; thus, after this operation, they are exactly $g(g-1)$ vertical edges, one for each of the columns. Finally, we repeatedly delete degree-1 vertices and suppress degree-2 vertices (Figure 9). This finishes the definition of the graph $G^{*}$. It is immediate to verify that $G^{*}$ contains the $(g \times g)$-grid as a minor. To finish the proof, it is enough to show that graph $G$ contains a subdivision of $G^{*}$. Each row $i$ corresponding to a horizontal path of $G^{*}$ is mapped to the path $Q_{i}$. Each vertical edge of $G^{*}$ will be mapped to a path in one of the even clusters as described in the following. We denote the vertical edges of $G^{*}$ by $e_{0}, e_{1}, \ldots, e_{g(g-1)-1}$, where $e_{i}$ is an edge that was lying on column $C_{i}$ of the grid. For each $0 \leq i<g(g-1)$, let $v_{i}, u_{i}$ be the two endpoints of $e_{i}$ in $G^{*}$; let $i_{1}$ be the index of the row to which $v_{i}$ belongs, and assume without loss of generality that $u_{i}$ belongs to row $\left(i_{1}+1\right)$. Let $P=Q_{i_{1}}$, the path to which row $i_{1}$ is mapped, and let $P^{\prime}=Q_{i_{1}+1}$, the path to which row $i_{1}+1$ is mapped. In the graph $G\left[S_{2(i+1)}\right]$, there is path $\beta_{2(i+1)}\left(P, P^{\prime}\right)$ connecting a vertex $a$ lying on $P$ to a vertex $b$ lying on path $P^{\prime}$. We map the edge $e_{i}$ to path $\beta_{2(i+1)}\left(P, P^{\prime}\right)$, vertex $v_{i}$ to $a$, and $u_{i}$ to $b$. Once we complete the mapping of all vertices and vertical edges of $G^{*}$, for every horizontal edge $e$ that lies say on the $j$ th row of $G^{*}$, we obtain a natural mapping of $e$ to the segment of $Q_{j}$ between the two vertices to which the endpoints of $e$ are mapped.

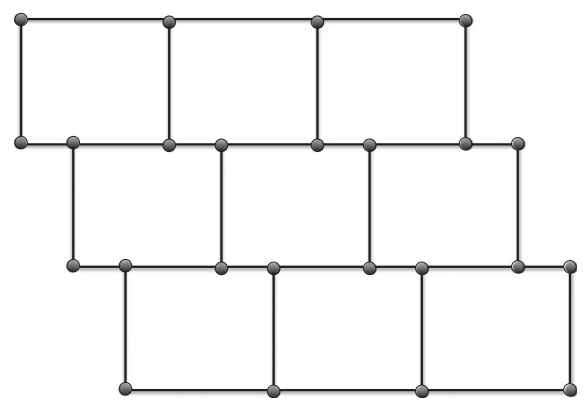

Fig. 9. Graph $G^{*}$ for $g=4$. 


\section{ACKNOWLEDGMENTS}

We thank Tasos Sidiropoulos for suggesting the problem of improving the bounds in the grid-minor theorem, and in particular for asking whether the ideas in routing algorithms are useful for this purpose. We also thank the two reviewers for detailed comments and suggestions.

\section{REFERENCES}

Matthew Andrews. 2010. Approximation algorithms for the edge-disjoint paths problem via Raecke decompositions. In Proceedings of the 2010 IEEE 51st Annual Symposium on Foundations of Computer Science (FOCS'10). IEEE, Los Alamitos, CA, 277-286. DOI : http://dx.doi.org/10.1109/FOCS.2010.33

Sanjeev Arora, Satish Rao, and Umesh Vazirani. 2009. Expander flows, geometric embeddings and graph partitioning. Journal of the ACM 56, 2, Article No. 5. DOI : http://dx.doi.org/10.1145/1502793.1502794

Chandra Chekuri and Julia Chuzhoy. 2013. Large-treewidth graph decompositions and applications. In Proceedings of the 45th Annual ACM Symposium on Theory of Computing (STOC'13). ACM, New York, NY, 291-300. DOI : http://dx.doi.org/10.1145/2488608.2488645

Chandra Chekuri and Julia Chuzhoy. 2015. Degree-3 treewidth sparsifiers. In Proceedings of the 26th Annual ACM-SIAM Symposium on Discrete Algorithms (SODA'15). 242-255. http://dl.acm.org/citation. cfm?id=2722129.2722148

Chandra Chekuri and Alina Ene. 2013. Poly-logarithmic approximation for maximum node disjoint paths with constant congestion. In Proceedings of the 24th Annual ACM-SIAM Symposium on Discrete Algorithms (SODA'13). 326-341. http://dl.acm.org/citation.cfm?id=2627817.2627841

Chandra Chekuri, Sanjeev Khanna, and F. Bruce Shepherd. 2005. Multicommodity flow, well-linked terminals, and routing problems. In Proceedings of the 37th Annual ACM Symposium on Theory of Computing (STOC'05). ACM, New York, NY, 183-192. DOI : http://dx.doi.org/10.1145/1060590.1060618

Chandra Chekuri, Sanjeev Khanna, and F. Bruce Shepherd. 2013a. The all-or-nothing multicommodity flow problem. SIAM Journal on Computing 42, 4, 1467-1493. DOI : http://dx.doi.org/10.1137/100796820

Chandra Chekuri and Nitish Korula. 2009. A graph reduction step preserving element-connectivity and applications. In Automata, Languages and Programming. Lecture Notes in Computer Science, Vol. 5555. Springer, 254-265. DOI : http://dx.doi.org/10.1007/978-3-642-02927-1_22

Chandra Chekuri, Guyslain Naves, and F. Bruce Shepherd. 2013b. Maximum edge-disjoint paths in $k$-sums of graphs. arXiv:1303.4897.

Julia Chuzhoy. 2015. Excluded grid theorem: Improved and simplified. In Proceedings of the 47th Annual ACM Symposium on Theory of Computing (STOC'15). ACM, New York, NY, 645-654. DOI : http://dx.doi.org/10.1145/2746539.2746551

Julia Chuzhoy. 2016. Routing in undirected graphs with constant congestion. SIAM Journal on Computing 45, 4, 1490-1532. DOI : http://dx.doi.org/10.1137/130910464

Julia Chuzhoy and Shi Li. 2016. A polylogarithmic approximation algorithm for edge-disjoint paths with congestion 2. Journal of the ACM (JACM) 63, 5, Article No. 45.

M. Conforti, R. Hassin, and R. Ravi. 2003. Reconstructing edge-disjoint paths. Operations Research Letters 31, 4, 273-276.

Erik Demaine, Mohammad Taghi Hajiaghayi, and Ken-Ichi Kawarabayashi. 2009. Algorithmic graph minor theory: Improved grid minor bounds and Wagners contraction. Algorithmica 54, 2, $142-180$. http://dx.doi.org/10.1007/s00453-007-9138-y

Erik D. Demaine, Fedor V. Fomin, Mohammad Taghi Hajiaghayi, and Dimitrios M. Thilikos. 2005. Subexponential parameterized algorithms on bounded-genus graphs and $H$-minor-free graphs. Journal of the ACM 52, 6, 866-893.

Erik D. Demaine and Mohammad Taghi Hajiaghayi. 2008. Linearity of grid minors in treewidth with applications through bidimensionality. Combinatorica 28, 1, 19-36.

Reinhard Diestel. 2012. Graph Theory (4th ed.). Graduate Texts in Mathematics, Vol. 173. Springer.

Reinhard Diestel, Tommy R. Jensen, Konstantin Y. Gorbunov, and Carsten Thomassen. 1999. Highly connected sets and the excluded grid theorem. Journal of Combinatorial Theory, Series B 75, 1, 61-73.

Devdatt Dubhashi and Alessandro Panconesi. 2009. Concentration of Measure for the Analysis of Randomized Algorithms. Cambridge University Press, New York, NY.

A. Hajnal and E. Szemerédi. 1970. Proof of a conjecture of P. Erdos. Combinatorial Theory and Its Applications 2, 601-623.

H. R. Hind and O. Oellermann. 1996. Menger-type results for three or more vertices. Congressus Numerantium 113, 179-204. 
Kenichi Kawarabayashi and Yusuke Kobayashi. 2012. Linear min-max relation between the treewidth of H-minor-free graphs and its largest grid. In Proceedings of the 29th International Symposium on Theoretical Aspects of Computer Science (STACS'12). 278-289. DOI:http://dx.doi.org/10.4230/LIPIcs. STACS.2012.278

Rohit Khandekar, Satish Rao, and Umesh Vazirani. 2009. Graph partitioning using single commodity flows. Journal of the ACM 56, 4, Article No. 19. DOI : http://dx.doi.org/10.1145/1538902.1538903

Stephan Kreutzer and Siamak Tazari. 2010. On brambles, grid-like minors, and parameterized intractability of monadic second-order logic. In Proceedings of the 21st Annual ACM-SIAM Symposium on Discrete Algorithms (SODA'10). 354-364. http://dl.acm.org/citation.cfm?id=1873601.1873631

Alexander Leaf and Paul Seymour. 2015. Tree-width and planar minors. Journal of Combinatorial Theory, Series B 111, C, 38-53. DOI : http://dx.doi.org/10.1016/j.jctb.2014.09.003

W. Mader. 1978. A reduction method for edge connectivity in graphs. Annals of Discrete Mathematics 3, $145-164$.

Lorenzo Orecchia, Leonard J. Schulman, Umesh V. Vazirani, and Nisheeth K. Vishnoi. 2008. On partitioning graphs via single commodity flows. In Proceedings of the 40th Annual ACM Symposium on Theory of Computing (STOC'08). ACM, New York, NY, 461-470. DOI : http://dx.doi.org/10.1145/1374376.1374442

Harald Räcke. 2002. Minimizing congestion in general networks. In Proceedings of the 43rd Symposium on Foundations of Computer Science (FOCS'02). IEEE, Los Alamitos, CA, 43-52. http://dl.acm.org/ citation.cfm?id=645413.652152

Satish Rao and Shuheng Zhou. 2010. Edge disjoint paths in moderately connected graphs. SIAM Journal on Computing 39, 5, 1856-1887.

Bruce Reed. 1997. Treewidth and tangles: A new connectivity measure and some applications. In Surveys in Combinatorics. Cambridge University Press, 87-162.

N. Robertson, P. Seymour, and R. Thomas. 1994. Quickly excluding a planar graph. Journal of Combinatorial Theory, Series B 62, 2, 323-348.

N. Robertson and P. D. Seymour. 1986. Graph minors. V. Excluding a planar graph. Journal of Combinatorial Theory, Series B 41, 1, 92-114.

Neil Robertson and Paul D. Seymour. 1991. Graph minors. X. Obstructions to tree-decomposition. Journal of Combinatorial Theory, Series B 52, 2, 153-190.

Alexander Schrijver. 2003. Combinatorial Optimization: Polyhedra and Efficiency. Algorithms and Combinatorics, Vol. 24. Springer.

Mohit Singh and Lap Chi Lau. 2015. Approximating minimum bounded degree spanning trees to within one of optimal. Journal of the ACM 62, 1, 1:1-1:19. DOI : http://dx.doi.org/10.1145/2629366

Sein Win. 1989. On a connection between the existence of $k$-trees and the toughness of a graph. Graphs and Combinatorics 5, 1, 201-205.

Received August 2014; revised July 2016; accepted September 2016 